\title{
ESSAYS ON CLIMATE ECONOMETRICS
}

\author{
A Dissertation \\ presented to \\ the Faculty of the Graduate School \\ at the University of Missouri-Columbia
}

In Partial Fulfillment

of the Requirements for the Degree

Doctor of Philosophy

\section{by \\ KYUNGSIK NAM}

Dr. J. Isaac Miller, Dissertation Supervisor

JULY 2018 
The undersigned, appointed by the Dean of the Graduate School, have examined the dissertation entitled:

\section{ESSAYS ON CLIMATE ECONOMETRICS}

presented by Kyungsik Nam, a candidate for the degree of Doctor of Philosophy and hereby certify that, in their opinion, it is worthy of acceptance.

Professor J. Isaac Miller

Professor David M. Kaplan

Professor Shawn Ni

Professor Christopher K. Wikle 
This dissertation is dedicated to my lovely wife, Yeji Lim. 


\section{ACKNOWLEDGMENTS}

My sincere appreciation should go to my dissertation advisor, Professor Zack Miller. Without his advice, I could not have started and finished these ambitious projects. Of course, there are a lot of his influences in this dissertation. It was a great honor for me to have a chance to work with him during one of the most precious time of my life. I also would like to express my appreciation to my other committee members, Professors David Kaplan, Shawn Ni, and Christopher K. Wikle for their thoughtful comments and suggestions. Also, I would like to extend my appreciation to good colleagues, Mr. Sanha Noh, Mr. Ingul Baek, and Mr. Taehwan Kim. They always have been willing to give me their best suggestions while circling the Francis Quadrangle, the historical center of the University of Missouri in Columbia.

Above all, I would like to thank my parents. My father, Dongwoo Nam and my mother, Kyungsun Park have supported and encouraged me to continue my doctoral work. Lastly, I am deeply grateful to my lovely wife, Yeji Lim for her endless support, encouragement, and prayers. Without her support, I could not even start my Ph.D. study. She motivated and encouraged me at the most frustrating moments. I would like to dedicate my dissertation to her.

Kyungsik Nam

in the McAlester Office

July 2018 


\section{CONTENTS}

ACKNOWLEDGMENTS

LIST OF TABLES $\quad$ iv

LIST OF FIGURES $\quad$ v

ABSTRACT viii

Chapter 1: Introduction 1

Chapter 1: Climatological Analysis $\quad 6$

Chapter 1: Econometric Model and Methodology 12

Chapter 1: Data 24

$\begin{array}{ll}\text { Chapter 1: Empirical Analysis } & 27\end{array}$

Chapter 1: Concluding Remarks

Chapter 1: References $\quad 36$

Chapter 2: Introduction $\quad 42$

Chapter 2: Econometric Model and Methodology 47

Chapter 2: The ENSO Dynamics and Its Uncertainty 56

Chapter 2: The Global Commodity Markets and Identifying Their Commonality 73

Chapter 2: Empirical Results 88

Chapter 2: Concluding Remarks 104

$\begin{array}{ll}\text { Chapter 2: References } & 105\end{array}$

Chapter 2: Appendices 113

$\begin{array}{ll}\text { VITA } & 126\end{array}$ 


\section{List of Tables}

1 Estimation results . . . . . . . . . . . . . . . . . . 28

$2 \quad \mathrm{AIC}$ and BIC values of each macroeconomic and the ENSO variables $\quad 60$

$3 \quad$ ERS AR(4) estimates for deseasonalized SOI and SST anomaly . . . 68

4 Correlation Matrix . . . . . . . . . . . . . . . 81

563 Commodity Prices (Cont.) . . . . . . . . . . . . . . . 124

$6 \quad 63$ Commodity Prices . . . . . . . . . . . . . . . . 125 


\section{List of Figures}

1 Comparisons of mean temperature anomalies for Globe/NH/SH . . . 25

2 Estimated GMT versus $\mathrm{TRF} \ldots \ldots . \ldots 26$

3 Nonlinear response function $B(r)$ and $d_{T}(r)$ for Globe/Southern Hemi-

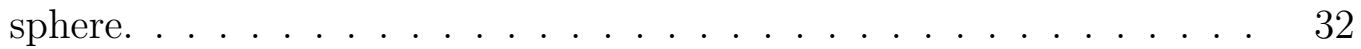

4 TRF response function to mean temperature anomalies for Globe/Southern

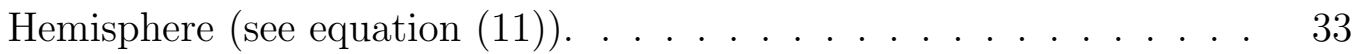

5 The Correlogram of each macroeconomic factor and SOI and SST anomaly data (clockwise) . . . . . . . . . . . . . 62

6 Deseasonalized SST anomaly and SOI data, their extracted factors, and profile likelihood surface and spectral densities for the deseasonalized

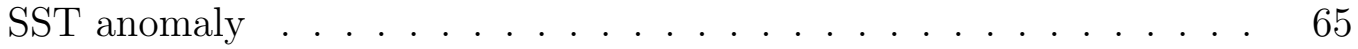

7 Climate Uncertainty with $68 \%$ Error Bands . . . . . . . . . . . . 70

$8 \quad$ ENSO Index . . . . . . . . . . . . . . . . . . . 71

$9 \quad R^{2}$ Comparison calculated from Dynamic PCA and Static PCA . . . 77

10 The Comparison between the first three static factors and the median of the posterior distribution of the common factors . . . . . . . 78

11 Top panel: the First Three Static Factors; Middle panel: the U.S. Exchange Rate Return, Global Commodity Inflation rate, Crude Oil Return; Bottom panel: the U.S. Industrial Production Growth Rate, the Change in 3 Month T-bill Rate, and deseasonalized SOI from 1992M1 to $2017 \mathrm{M} 6 \ldots \ldots \ldots \ldots$

12 Rolling $R^{2}$ Statistics for the First Three Static Factors from All De-

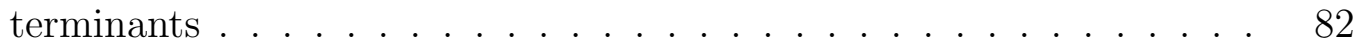

13 MC Statistics of the First Factor from Each Determinant . . . . . . . 83

14 MC Statistics of the Second Factor from Each Determinant . . . . . . 84

15 MC Statistics of the Third Factor from Each Determinant . . . . . . 84 
16 Time-varying Contribution of Each Determinant on the Global Commodity Markets .................. 86

17 Market Uncertainty with $68 \%$ Error Bands . . . . . . . . . . . . . 87

18 The spatially-heterogeneous ENSO effects . . . . . . . . . . . 89

19 The selected three dimensional median impulse responses to a climate uncertainty shock . . . . . . . . . . . . . . . . . 91

20 The yearly averaged median impulse responses to a climate uncertainty shock at selected years . . . . . . . . . . . . . . . . . . . 92

21 The time-varying median impulse responses of Maize (left column) and Soybean (right column) inflation rate to a climate uncertainty shock at horizons 1-5 with $68 \%$ error bands . . . . . . . . . . . . . . . 96

22 The selected median impulse responses to a market uncertainty shock $\quad 97$

23 The yearly averaged median impulse responses to a market uncertainty shock at selected years . . . . . . . . . . . . . . . . . . . 98

24 The median impulse responses of Gold inflation rate to a climate uncertainty shock (left column) and those to a market uncertainty shock (right column) . . . . . . . . . . . . . . . .

25 The median impulse responses of Arabica coffee inflation rate to a climate uncertainty shock (left column) and those to a market uncertainty shock (right column) . . . . . . . . . . . . . . . 102

26 The median impulse responses of Robusta coffee inflation rate to a climate uncertainty shock (left column) and those to a market uncertainty shock (right column) . . . . . . . . . . . . . . . 103

27 The 4-5 year cyclical (left) and high frequency cycle (right) components of the SST anomaly . . . . . . . . . . . . . . . . . . 119 
28 The estimate of climate uncertainty from the fully specified model and that from the model with no cyclical terms and that from the FAVAR with uncertainty model and ERS generated data . . . . . . . . . 120

29 The unobserved component and first static principal component and their sample autocorrelation . . . . . . . . . . . . . . . . . 122

30 Convergence check of the MCMC algorithm . . . . . . . . . . 123 


\section{ABSTRACT}

\section{Chapter 1: Nonlinear Cointegrating Regression of Earth's Surface Mean}

\section{Temperature Anomalies on Total Radiative Forcing}

This paper proposes a nonlinear cointegrating regression model based on the well-known energy balance climate model. Specifically, I investigate the nonlinear cointegrating regression of mean of temperature anomaly distributions on total radiative forcing using estimated spatial distributions of temperature anomalies for Globe/Northern Hemisphere/Southern Hemisphere. Further, I provide two types of nonlinear response functions that map from the total radiative forcing level to mean temperature anomalies. Cointegration and specification tests are also provided that support the existence of nonlinear effects of total radiative forcing.

\section{Chapter 2: Dynamic Factors and Climate Uncertainty in Global Commod- ity Market}

This paper investigates the effect of climate uncertainty on global commodity markets. To do so, I modify a time-varying factor-augmented VAR (FAVAR) with stochastic volatility in mean model of Mumtaz and Theodoridis (2018). By incorporating the information from a large data set with an efficiently constructed dynamic factor structure, I not only overcome the omitted variable problem but also maintain the efficiency of the estimator to identify the nonlinear climate effects. Moreover, I apply the endogenous regime switching in mean model of Chang et al. (2017) for the climate variable, in order to overcome the statistical problem generated by the periodicity. Lastly, I identify the commonality of the global commodity markets using the classical principal component analyses. In doing so, I reveal an economic mechanism that causes the omitted variable problem. The main empirical results can be summarized as follows. First, the climate uncertainty generates an inflationary pressure to agricultural food, non-energy, and energy commodities except for metal \& mineral 
item. Second, the individual items such as maize and soybeans are more sensitive than the aggregated commodity indices from the impact of the climate uncertainty. Third, the climate uncertainty generates a negative supply shock while the global market uncertainty generates a negative demand shock for the individual agricultural items. 


\section{CHAPTER 1}

\section{Nonlinear Cointegrating Regression of Earth's Surface Mean Temperature Anomalies on Total Radiative Forcing}

\section{Introduction}

OBSERVED GLOBAL MEAN SURFACE TEMPERATURE has been increasing over the past decades. ${ }^{1}$ With such an evident fact, global warming has received rapidly growing attention over the past decade. Since all countries are involved in both causes and consequences in a variety of complex ways, there have been world-wide debates on global warming among scientists and policy makers. To identify whether human activities cause the recent rising in global mean temperature or whether their effects will bring serious effects on the Earth, the detection and attribution of an anthropogenic influence on climate change have been extensively studied.

Two scientific theories must be recognized. The first scientific theory is the greenhouse effect. Ultraviolet light from the Sun passes through the greenhouse gases (GHG) such as carbon dioxide, water vapor, methane, nitrous oxide and chlorofluorocarbons and is absorbed by objects on the ground. The problem is the energy that the objects release is infrared radiation. Since the greenhouse gases absorb the infrared radiation and then reradiates it back to the surface of the Earth, the global temperature is increasing. We call this the "Greenhouse Effect" (Hansen et al., 2011). The second scientific theory is that the greenhouse gases in the atmosphere have been consistently increasing due to human activities. Note that the atmospheric lifetime of

\footnotetext{
${ }^{1}$ The reader is referred to Miller and Nam (2017) for the hiatus attribution study.
} 
the carbon dioxide is currently estimated by 5-200 years (IPCC, 2007). As a result of such accumulation, the stock of carbon dioxide in the atmosphere has been increased by about $130 \mathrm{ppm}$ over the last 270 years, from a range of 275 to $285 \mathrm{ppm}$ in the pre-industrial era (AD 1750) to 410 ppm in 2018.

According to the Intergovernmental Panel on Climate Change (2014), the global mean temperature on the surface of the Earth has increased about $0.85^{\circ} \mathrm{C}$ since 1880 and "most of the observed warming over the last 50 years is likely to have been due to the increase in greenhouse gas emissions." Scientists have attempted to estimate the effect of greenhouse gases. Their estimates tell us that the doubling carbon dioxide concentration in the atmosphere (a forcing of $4 \mathrm{~W} / \mathrm{m}^{2}$ ) may increase the global average temperature by 1.5 to $4.5^{\circ} \mathrm{C}$. To stress the serious impact from increasing global temperature, Stern (2008) states, "Around 10,000-12,000 years ago, temperatures were around $5^{\circ} \mathrm{C}$ lower than today, and ice sheets came down to latitudes just north of London and just south of New York. As the ice melted and sea levels rose, England separated from the continent, rerouting much of the river flow. These magnitudes of temperature changes transform the planet." However, there is no clear consensus when the carbon dioxide concentration in the atmosphere would be doubled.

Time-series analysis has been employed to test the anthropogenic global warming hypothesis. There has been extensive controversy on this hypothesis test over the last two decades. The main argument on the controversy is whether observing trends in temperature series and radiative forcing contain stochastic trends or deterministic trends with a structural break. At an early stage, Stern and Kaufmann (1997, 1999), Kaufmann and Stern $(1997,2002)$ and Kaufmann et al. (2006) provided a breakthrough on the linear cointegration analysis between temperature series and radiative forcing variables by assuming them as integrated processes or difference stationary processes. Using Dynamic Ordinary Least Squares (DOLS) estimation, they conclude that the increase in global mean temperature can be associated with the 
changes in radiative forcing variables. Such a linear cointegration analysis is also investigated by Pretis (2015). He linked two-component energy balance climate model of global mean temperature with a testable cointegrated VAR.

Some econometricians cast doubt on their statistical rigor and challenge their empirical results (Estrada and Perron 2012, 2014; Estrada et al., 2013; Gay et al., 2009). They start with arguing that temperatures and radiative forcing variables are better described as trend stationary processes rather than difference stationary processes (or random walk with drift). By defining variables of interest as stationary processes fluctuating around a common breaking deterministic trend, they argue that the conventional LS method on the regression may cancel out a common feature as in cointegration analysis. Moreover, they argue that the residual-based ADF test (or formally nonparametric nonlinear co-trending test of Bierens, 2000) may identify the existence of long-run relationship.

In addition to discussions of trend characteristics, the direction of causality is also an issue among the climate scientist. With Vostok Ice-Core (VIC) long-term temperature data for the last 420,000 years (Petit et al., 2001), there has been a high correlation between atmospheric $\mathrm{CO}_{2}$ concentration and temperatures and sea level through the four glacial-interglacial cycles. However, their causal direction is the reverse of what the conventional climate models postulate. Specifically, the distribution of sunlight on Earth has been changed by small changes in Earth's orbit over hundreds of thousands of years. Melting ice sheets due to more sunlight decrease the Earth's surface albedo and make the Earth warmer. As a consequence, an increase in atmospheric $\mathrm{CO}_{2}$ concentration can be the outcome of temperature rising because warming natural reservoirs like ocean release $\mathrm{CO}_{2}$ to the atmosphere (Hansen and Sato, 2012).

Further, carbon storage in the oceans can also be affected by ocean circulation. The decline in latitudinal overturning oceans (Ocean Conveyor Belt) as a result of 
global warming slows down absorbing of the atmospheric $\mathrm{CO}_{2}$. Such nonlinear positive feedbacks enhance the increase in atmospheric $\mathrm{CO}_{2}$ concentration. Although described time span is too long to apply for currently available data, the bidirectional causality seems to be the most reasonable expression for the relationship between atmospheric $\mathrm{CO}_{2}$ concentration and temperatures and sea level.

Recently, Chang et al. (2016b) analyze the global warming issue under a novel time-series framework. With Global/Northern Hemisphere/Southern Hemisphere temperature anomaly data from 1850 to 2012, they generate the distributions of temperature anomalies for each year. Instead of analyzing only the mean temperature anomalies, they analyze and test the persistent features of distributions of temperature anomalies by regarding them as functional time-series observations on the Hilbert space. More importantly, they distinguish the unit-root nonstationarity from the deterministic or explosive nonstationarity in their testing procedure (Chang et al., 2016a, 2016b). They conclude that the first few of moments of temperature anomaly distribution have stochastic trends, rather than linear/exponential/quadratic trend or explosive roots.

In particular, they argue that the seemingly structural break in the trend of global mean temperature anomaly, which is argued by many authors, could be inherited more likely from unit-root type persistency (stochastic trend), than from higher order persistency associated with deterministic trends. Based on their analysis, the global temperature anomaly distribution and radiative forcing variables can share common stochastic trends and their linear combination can produce a stationary process. In this context, the nonlinear cointegration analysis, which allows for bidirectional causality and postulates long-run equilibrium relationship between mean temperature anomalies and total radiative forcing and possibly with nonlinear moments of temperature anomaly distributions, seems to be the most reasonable approach.

More importantly, the linear regression model, which analyzes the linear relation- 
ship between global mean temperature and total radiative forcing variable, does not have a climatological mechanism about how to change the global mean temperature. Since the atmospheric $\mathrm{CO}_{2}$ increases global temperature by generating imbalanced energy equation, a channel for that greenhouse effect must be considered. As I will show in Section 2, the climate channel is represented as temperature dependent net incoming absorbed radiation, implying that the relationship between the total radiative forcing and global mean temperature could be expressed as a temperature heterogeneous function. Obviously, ignoring an omitted term, the net incoming absorbed radiation generates an endogeneity problem, invalidating a slope estimator in the linear model.

Throughtout this paper, I aim to provide a new econometric methodology to better explain nonlinear dependence between climate variables. To do so, I propose the nonlinear cointegrating regression of Earth's surface mean temperature anomalies on total radiative forcing variable under technical backgrounds provided in Chang et al. (2016b). Put differently, I analyze the cointegrating relationship between times series of distributions of temperature anomalies and time series of total radiative forcing level. I also define the misspecification error from the nonlinear cointegration model and identify the source of error in terms of temperature anomaly. Further, I provide the cointegration and specification tests that support the existence of nonlinear effects of total radiative forcing. In a climatological sense, the proposed econometric methodology provides two types of the nonlinear response functions that map from the total radiative forcing to mean temperature anomalies for Globe/Northern Hemisphere/Southern Hemisphere, which take account of the temperature dependent net incoming absorbed radiation. Specifically, I explicitly estimate the nonlinear effects by defining the nonlinear temperature terms from the temperature anomaly distributions. I call the nonlinear response function the temperature-dependent effect of the total radiative forcing with assuming that the net incoming absorbed radiation is 
hypothetically and solely determined by a temperature anomaly.

The rest of the paper is organized as follows. Section 2 provides the climatological background with global energy balance climate model. Section 3 presents the econometric model and methodology. Specifically, I employ the functional coefficient model for a nonlinear cointegrating regression model and applied it to the current climate model. In Section 4, I discuss the details of data. In Section 5, I present empirical results and provide interpretations on the presented results, and I conclude with Section 6 .

\section{Climatological Analysis}

\subsection{Global Energy Balance Climate Model}

North et al. (1983) developed the two-dimensional energy balance climate model (hereafter EBCM) and Brock et al. (2013) consider their model with human relating forcing activity. The EBCM model that is recently provided by Brock et al. (2013) is given by

$$
C(\hat{r}) \frac{\partial s_{\hat{r}, t}}{\partial t}=\underbrace{Q S(x) \alpha\left(x, s_{\hat{r}, t}\right)}_{\text {Incoming absorbed radiation }}+\underbrace{h_{\hat{r}, t}-I_{\hat{r}, t}}_{(-) \text {Outgoing radiation }}+\underbrace{\nabla\left[D(x) \nabla s_{\hat{r}, t}\right]}_{\text {Divergence in heat flux }}
$$

where $\hat{r}=(\theta, \phi)$ is the point on the surface, where $\theta \in\left[-\frac{\pi}{2}, \frac{\pi}{2}\right]$ is latitude and $\phi \in[0,2 \pi]$ is longitude, $C(\hat{r})$ is effective local heat capacity per unit area, and $s_{\hat{r}, t}$ is the Earth surface temperature at location $\hat{r}$ and time $t$. By defining $x=\sin \theta$, the incoming solar radiation hitting the surface of the Earth is $Q S(x) \alpha\left(x, s_{\hat{r}, t}\right)$, where $Q$ is solar constant divided by $4, S(x)$ is mean annual meridional distribution of solar radiation, in which its integral from -1 to 1 must be unity, and $\alpha\left(x, s_{\hat{r}, t}\right)$ is absorption coefficient or co-albedo function, which is one minus the albedo of the Earth-atmosphere system. The outgoing radiation is $I_{\hat{r}, t}$, which is determined by 
surface temperature. The total radiative forcing component at specific location $\hat{r}$ and time $t$ is $h_{\hat{r}, t}$, which includes the human related activities influencing the climate. ${ }^{2}$ $D(x)$ is diffusion term for all different forms of heat transport. The heat transport (divergence in heat flux) term, $\nabla\left[D(x) \nabla s_{\hat{r}, t}\right]$, is due the incoming absorbed radiant heat not being matched by the net outgoing radiation.

As will be explained in Data section, the global temperature data is expressed as anomalies from the zero-base period. ${ }^{3}$ To take into account the data characteristics, I consider the discretized EBCM model at specific location $\hat{r}$ and time $t$ as given by

$$
C_{\hat{r}}\left(s_{\hat{r}, t}-s_{\hat{r}, 0}\right)=Q S(x) \alpha\left(x, s_{\hat{r}, t}\right)+h_{\hat{r}, t}-O_{\hat{r}} s_{\hat{r}, t}+\nabla\left[D(x) \nabla s_{\hat{r}, t}\right]
$$

where $\hat{r}=(x, \phi)$ instead of $\hat{r}=(\theta, \phi)$ for simplicity and $s_{\hat{r}, 0}$ is mean temperature at location $\hat{r}$ during the zero-base period, $[\underline{t}, \bar{t}]$. Note that the outgoing radiation term, $I_{\hat{r}, t}$ can be replaced with empirical formula, $O_{\hat{r}} s_{\hat{r}, t}$ (Budyko, 1969). Throughout this paper, I consider the Energy Balance Climate Model of the entire surface area of the Earth by integrating over $\hat{r}$ (so called Global Energy Balance Climate Model),

$$
\begin{aligned}
\int_{-1}^{1} \int_{0}^{2 \pi} C_{\hat{r}}\left(s_{\hat{r}, t}-s_{\hat{r}, 0}\right) d \phi d x= & \underbrace{\int_{-1}^{1} \int_{0}^{2 \pi} Q S(x) \alpha\left(x, s_{\hat{r}, t}\right) d \phi d x}_{\text {Incoming absorbed radiation }}-\underbrace{\int_{-1}^{1} \int_{0}^{2 \pi} O_{\hat{r}} s_{\hat{r}, t} d \phi d x}_{\text {Outgoing radiation }} \\
& +\underbrace{\int_{-1}^{1} \int_{0}^{2 \pi} h_{\hat{r}, t} d \phi d x}_{\text {Total radiative forcing }}+\underbrace{\int_{-1}^{1} \int_{0}^{2 \pi} \nabla\left[D(x) \nabla s_{\hat{r}, t}\right] d \phi d x}_{\text {Divergence in heat flux }}
\end{aligned}
$$

Note that the last term, $\int_{-1}^{1} \int_{0}^{2 \pi} \nabla\left[D(x) \nabla s_{\hat{r}, t}\right] d \phi d x$ is assumed to be zero because heat transports (typically from low to high latitudes) are cancelled out across the entire surface area of the Earth. Moreover, dividing $4 \pi$ on both sides of equation (1)

\footnotetext{
${ }^{2}$ Brock et al. (2013) consider only atmospheric $\mathrm{CO}_{2}$ concentration, in place of $h_{\hat{r}, t}$.

${ }^{3}$ For GISS dataset, the zero-base period is from 1951M1 to $1980 \mathrm{M} 12$.
} 
provides an interpretation that global mean temperature anomaly is net incoming absorbed radiation across the globe plus total radiative forcing.

Although integrating divergence in heat flux across the globe is zero, there are spatial heterogeneities in net incoming absorbed radiation across the globe. Assuming a constant heat capacity (i.e., $C_{\hat{r}}=C$ for all $\left.\hat{r}\right)^{4}$ in equation (1) provides

$$
\begin{aligned}
C\left(s_{t}-s_{0}\right) & =\frac{1}{4 \pi} \int_{-1}^{1} \int_{0}^{2 \pi}\left(Q S(x) \alpha\left(x, s_{\hat{r}, t}\right)-O_{\hat{r}} s_{\hat{r}, t}+h_{\hat{r}, t}\right) d \phi d x \\
& =\frac{1}{4 \pi} \int_{-1}^{1} \int_{0}^{2 \pi}\left(Q S(x) \alpha\left(x, s_{\hat{r}, t}\right)-O_{\hat{r}} s_{\hat{r}, t}\right) d \phi d x+h_{t} \\
& \equiv B_{t}\left(s_{\hat{r}, t}\right)+h_{t}
\end{aligned}
$$

where

$$
B_{t}\left(s_{\hat{r}, t}\right)=\frac{1}{4 \pi} \int_{-1}^{1} \int_{0}^{2 \pi}\left(Q S(x) \alpha\left(x, s_{\hat{r}, t}\right)-O_{\hat{r}} s_{\hat{r}, t}\right) d \phi d x, \quad h_{t}=\frac{1}{4 \pi} \int_{-1}^{1} \int_{0}^{2 \pi} h_{\hat{r}, t} d \phi d x
$$

and

$$
s_{t}-s_{0}=\frac{1}{4 \pi} \int_{-1}^{1} \int_{0}^{2 \pi}\left(s_{\hat{r}, t}-s_{\hat{r}, 0}\right) d \phi d x
$$

Note that $s_{t}$ is global mean temperature at time $t, s_{0}$ is global mean temperature during the zero-base period, $[\underline{t}, \bar{t}]$ and therefore $s_{t}-s_{0}$ is global mean temperature anomaly at time $t$. Further note that integrating local temperatures across $x=\sin \theta$ provides a way that temperatures in low latitudes have more weights in calculating the global mean temperature $s_{t}$, so that the increase in temperature at high latitudes due to divergence in heat flux from low to high latitudes is roughly proportional to the temperature difference between high and low latitudes (Held and Suarez, 1974

\footnotetext{
${ }^{4} \mathrm{I}$ acknowledge that the constant heat capacity is too restrictive assumption. Since the goal of this paper is to develop the statistical model based on underlying physics, and the non-constant heat capacity makes the model too complicated, I assume a phenomenological constant heat capacity (Brock et al., 2013). However, this issue is addressed in another working paper (see Miller and Nam, 2017).
} 
for one-dimensional EBCM model).

Equation (2) tells us that spatially distributed total radiative forcing level along with different spatial characteristics over the Earth's atmosphere creates spatially heterogeneous warming effects. Consequently, the global EBCM tells us that spatially averaged net incoming absorbed radiation could be proportional to the global mean temperature anomaly we observed through the data. More specifically, outgoing radiation to space is reduced by total radiative forcing level at each location (greenhouse effect) and that the discrepancies between incoming radiation and outgoing radiation that is offset by exogenous forcings such as human and volcano activities (without considering divergence in heat flux) generate historical global mean temperature anomaly data. In other words, the global mean temperature anomaly is determined by spatially heterogeneous net incoming absorbed radiation, which is a function of local temperatures, $s_{\hat{r}, t}$, as well as total radiative forcing level in a complex way.

\subsection{Nonlinear Relationship between Total Radiative Forcing Level and Global Mean Temperature Anomaly}

In this section, I analytically show the nonlinear dependence of the total radiative forcing (hereafter, TRF) on global mean temperature anomaly. The (transient) Climate Sensitivity is often expressed as the change in temperature associated with a doubling of the concentration of carbon dioxide in Earth's atmosphere, which is likely to be in the range 1 to $2.5^{\circ} \mathrm{C}$ (Bindoff et al., 2013). Technically speaking, however, the Climate Sensitivity (CS) measures the change in eventual (equilibrium) global surface mean temperature $\left(\Delta s_{e q}\right)$ per unit of total radiative forcing (Hansen et al., 
2013), which is defined as

$$
C S=\frac{\Delta s_{e q}}{h}
$$

More importantly, the word, "equilibrium", imposes a notion of the length of time between two equilibrium climate states, which are the climate state in when the unit of total radiative forcing bumps the energy budget out of balance, and the climate state in when the Earth's energy balance system is restored. In doing so, the climate sensitivity measures the difference in two Earth's surface mean temperatures that compose the Earth's energy balance body in different time. Note that since global surface temperature cannot respond to the total radiative forcing or other climate forcing variables instantaneously due to thermal inertia of the climate system, the restricted data span lets the estimate of a climate sensitivity fail to take account of slow feedback processes.

To estimate a constant climate sensitivity parameter, numerous authors estimate the slope of the following linear model: Stern and Kaufmann $(1997,1999)$ and Estrada et al., (2013), inter alia.

$$
s_{t}-s_{0}=\text { constant }+ \text { slope } \times h_{t}
$$

Note that slope estimate indicates that the change in global surface mean temperature anomaly with respect to unit change in TRF and is expressed in units of ${ }^{\circ} \mathrm{C} /\left(\mathrm{W} / \mathrm{m}^{2}\right)$, and therefore the estimation model (4) postulates that the TRF effects are heterogeneous across the evaluated point, unlike the defined climate sensitivity in (3). More importantly, there is an endogeneity problem, inherited from the omitted term, $B_{t}\left(s_{\hat{r}, t}\right)$ in estimating equation (4), implying the invalidity of a slope estimator. Specifically, the reduced form regression model for structural equation (2) cannot be simply estimated or evaluated, because two terms, $B_{t}\left(s_{\hat{r}, t}\right)$ and $h_{t}$, are intertwined 
physically. To produce physically sounded estimates, there must be a channel that the TRF level, $h_{t}$, affects the global mean temperature through net incoming absorbed radiation, $B_{t}\left(s_{\hat{r}, t}\right)$, (indirect effect) because the TRF affects global temperature by generating imbalanced energy equation. In the absence of net incoming absorbed radiation term, however, a constant TRF effect is evaluated at all temperature anomalies, assuming a linear dependence structure between global mean temperature anomaly and TRF. In this vein, taking account of omitted term, $B_{t}\left(s_{\hat{r}, t}\right)$ in the evaluating the climate sensitivity is required. Note that the net incoming absorbed radiation is not observable. Here, I consider that the TRF level affects the whole temperature anomaly distribution and that nonlinear temperature terms (or higher order moments of temperature anomaly distribution), which are defined as distributional temperature terms minus a linear temperature term, are regarded as information for net incoming absorbed radiation. Further, I assume that the TRF affects net incoming absorbed radiation in temperature-heterogeneous fashion.

In the estimation procedure, I first identify a constant (linear) TRF effect on global mean temperature anomaly and then estimate the remaining nonlinear TRF effect at each temperature anomaly. Climatologically, I may call the sum of constant linear effect and a nonlinear effect of TRF at a temperature anomaly, $r_{0}$, the temperaturedependent TRF effect (or the TRF response function) with assuming that the net incoming absorbed radiation is hypothetically and solely determined by a temperature anomaly, $r_{0}$. The positive gap between TRF response function and a linear TRF effect at some temperature anomaly therefore implies that their relationship given that anomaly is underestimated. The reader is referred to Miller (2017) for the advanced climatological modeling. ${ }^{5}$

\footnotetext{
${ }^{5}$ This paper focuses on developing reduced-form nonlinear cointegrating model based on energy balance climate model. However, Miller (2017) provides the climatological modeling with the first law of thermodynamics (i.e., integrating net incoming absorbed radiation across the globe is zero, but with non-zero derivatives).
} 


\section{Econometric Model and Methodology}

\subsection{Reverse Functional Coefficient Model}

In what follows, I propose the econometric procedures to estimate the greenhouse effect. The reverse functional coefficient model is a particular interest of this paper, as given by

$$
\int_{-\infty}^{\infty} B^{*}(r) f_{t}(r) d r=a_{0}+a_{1} h_{t}+\varepsilon_{t}
$$

where $r_{t}$ is the random variable representing the raw temperature anomaly and having density $f_{t}(r)$ and $h_{t}$ is TRF level. The interpretation of equation (5) is that TRF level can affect the whole temperature anomaly distribution with arbitrary fashion. Specifically, the TRF effect on temperature anomaly $r$ is measured as $B^{*}(r) / a_{1}$. Here, I assume that $B^{*}(r)$ can be approximated by a Functional Fourier Flexible (FFF) form as given by

$$
B_{p q}^{*}(r)=\sum_{j=1}^{p} b_{j}^{*} r^{j}+\sum_{j=1}^{q}\left(b_{p+2 j-1}^{*}, b_{p+2 j}^{*}\right) \phi_{j}(r)
$$

where $\phi_{j}(r)=(\cos 2 \pi j r, \sin 2 \pi j r)^{\prime}$ as introduced by Gallant (1981) and extensively exploited by Park et al. (2010) and Chang et al. (2014). At first, I decompose the left-hand side into the linear part, $b_{1}^{*} r$ and the nonlinear part, $B_{p q}^{*}(r)$, where $B_{p q}^{*}(r)=\sum_{j=2}^{p} b_{j}^{*} r^{j}+\sum_{j=1}^{q}\left(b_{p+2 j-1}^{*}, b_{p+2 j}^{*}\right) \phi_{j}(r)$.

Specifically, the linear part contains a term that the functional coefficient is linear in temperature anomaly $r$ and expressed as the mean temperature anomaly. On the other hand, the nonlinear part contains terms that the functional coefficient is nonlinear in temperature anomaly $r$. As a result, I have

$$
b_{1}^{*} \int_{-\infty}^{\infty} r f_{t}(r) d r+\int_{-\infty}^{\infty} B_{p q}^{*}(r) f_{t}(r) d r=a_{0}+a_{1} h_{t}+\varepsilon_{t}
$$


After rearranging equation (6), I have

$$
\int_{-\infty}^{\infty} r f_{t}(r) d r=b_{0}+b_{1} h_{t}+\int_{-\infty}^{\infty} B(r) f_{t}(r) d r+u_{t}
$$

where $B(r)=-B_{p q}^{*}(r) / b_{1}^{*}, b_{0}=a_{0} / b_{1}^{*}, b_{1}=a_{1} / b_{1}^{*}$, and $u_{t}=\varepsilon_{t} / b_{1}^{*}$. Note that equation (7) specifies the statistical relationship between global mean temperature anomaly (left hand side) and the TRF level (second term of right hand side) and sum of probability-weighted net incoming absorbed radiation (third term of right hand side). Specifically, I implicitly assume that the net incoming absorbed radiation, $B_{t}\left(s_{\hat{r}, t}\right)$, in global EBCM (2) is decomposed into linearly additive functions at each local temperature anomaly, $b(s)$, with associated probability weight, $f_{t}(s)$, which may be represented as

$$
B_{t}\left(s_{\hat{r}, t}\right)=\int_{s} b(s) f_{t}(s) d s \equiv \mathbb{E}\left[b\left(s_{t}\right)\right]
$$

In this light, the third term, $\int B(r) f_{t}(r) d r$, which is also expressed as $\mathbb{E}\left[B\left(r_{t}\right)\right]$, in equation (7) provides a channel for net incoming absorbed radiation, $B_{t}\left(s_{\hat{r}, t}\right)$, in global EBCM (2) and that term affects global mean temperature anomaly by TRF level through changed temperature probabilities. In this context, the reduced form model (7) corresponds to the global EBCM in equation (2). ${ }^{6}$

Statistically, equations (5) and (7) can be analyzed as a cointegrating relationship given by

$$
h_{t}-\beta_{p q}^{\prime} x_{p q t} \sim I(0)
$$

\footnotetext{
${ }^{6}$ Note that $s_{\hat{r}, t}$ in global $\operatorname{EBCM}(2)$ is observed temperature anomaly at location $\hat{r}$ and time $t$, and $r_{t}$ in reduced form equation (7) is temperature anomaly observed on the surface of Earth. Therefore, they are regarded as a same variable.
} 
where the multivariate time-series vector, $x_{p q t}=\left(\int r f_{t}(r) d r, \int r^{2} f_{t}(r) d r, \ldots\right.$, $\left.\int \phi_{j}(r) f_{t}(r) d r\right)^{\prime}$ with cointegrating vector, $\beta_{p q}=\left(1, c_{1}, c_{2}, \ldots, c_{p+2 q}\right)^{\prime}$. Note that mean-level regression implemented in the climate literature assumes that the TRF level only affects the aggregated temperature anomaly distribution towards the mean process. In this paper, however, I consider the cointegrating relationship between temperature anomaly distribution and the TRF level. More specifically, the relationship between them is the cointegrating relationship between several variables, which are global mean temperature anomaly, the TRF level, and the nonlinear temperature terms. Furthermore, the nonlinear cointegration analysis in this paper becomes the distributional cointegration analysis between temperature anomaly distribution and total radiative forcing distribution, if we assume that the total radiative forcing is distributed homogeneously across the Earth's atmosphere.

In particular, I provide two types of estimators from a nonlinear cointegration model. The first estimator, $D^{1}$, is to provide the slope estimator that takes into account the TRF effect on global mean temperature anomaly through nonlinear temperature terms. On the other hand, the second estimator, $D^{2}(r)$, is to provide the temperature-dependent slope estimator, which is illustrated through TRF response function. Statistically, the derivative $\partial\left(\int r f_{t}(r) d r\right) / \partial h_{t}$ - which is equal to $b_{1}$ in the linear case - estimates climate sensitivity. Suppose spatial temperature anomaly distributions $f_{t}(r)$ across the globe for all time $t$ are observed over the domain, $D_{r}=\left[\lambda_{1}, \lambda_{2}\right]$. Moreover, I consider the spatial temperature anomaly distributions themselves are considered as random variables under predetermined temperature anomalies $r$ 's. Under fixed temperature anomalies $r$ 's, the total derivatives of $f_{t}\left(r, h_{t}\right)$ with respect to $h_{t}$ become the partial derivatives of $f_{t}\left(r, h_{t}\right)$ with respect to $h_{t}$. Thus, 
we have

$$
\begin{aligned}
D^{1} \equiv \frac{\partial \int_{D_{r}} r f_{t}(r) d r}{\partial h_{t}} & =\int_{D_{r}} r \frac{\partial f_{t}(r)}{\partial h_{t}} d r=b_{1}+\int_{D_{r}} B(r) \frac{\partial f_{t}(r)}{\partial h_{t}} d r \\
& =b_{1}+\frac{\partial \int_{D_{r}} B(r) f_{t}(r) d r}{\partial h_{t}}
\end{aligned}
$$

Note that total differentiating equation (7) and replacing it with partial derivatives provides equation (10). As stated above, the last equality of equation (10) holds under fixed temperature anomalies (i.e., $\int \frac{\partial B(r)}{\partial h_{t}} f_{t}(r) d r=0$ ), which means that the temperature anomalies $r$ 's are invariant with respect to $h_{t}$. The probabilities at fixed temperature anomalies, however, are affected by $h_{t}$.

More importantly, the model (7) reduces to standard linear model in the climate literature when $B(r)=0$ (Estrada et al., 2013). If $B(r) \neq 0$ and I omit the term, $\int B(r) f_{t}(r) d r$, however, the regression equation (7) becomes a spurious regression and therefore the conventional test statistics on the estimator $\hat{b}_{1}$ would be invalid. Interestingly, by including a second term in equation (10), the temperature-dependent climate sensitivity can be identified and therefore the different TRF effects on global mean temperature anomaly at each anomaly are identified. Specifically, when the net incoming absorbed radiation is hypothetically determined at $r_{0} \in\left[\lambda_{1}, \lambda_{2}\right]$, the TRF effect on global mean temperature anomaly is estimated by

$$
D^{2}\left(r_{0}\right)=b_{1}+B\left(r_{0}\right) d_{T}\left(r_{0}\right)
$$

where

$$
d_{T}\left(r_{0}\right)=\left.\frac{\partial f_{t}(r)}{\partial h_{t}}\right|_{r=r_{0}}
$$

Note that $d_{T}(r)$ is estimated by linear regression of probability series at each temperature anomaly on TRF with a constant using least squares method. I call the second terms in equations (10) - (11) the nonlinear effect of TRF to global mean 
temperature anomaly. Subsequently, I define the misspecification error of the linear model, which represents error from ignoring the nonlinear effects, as

$$
\operatorname{Error}(r) \equiv b_{1}-D^{1}=-\frac{\partial \int_{D_{r}} B(r) f_{t}(r) d r}{\partial h_{t}}
$$

The sum of nonlinear effects of the estimator, $D^{2}(r)$, across temperature anomalies $r$ 's is equal to the nonlinear effect of the estimator, $D^{1}$. In this sense, error calculation from the estimator, $D^{2}(r)$, enables us to identify the contribution of misspecification error at each temperature anomaly, $r_{0} \in\left[\lambda_{1}, \lambda_{2}\right]$, which is represented as

$$
-\left.B\left(r_{0}\right) \frac{\partial f_{t}(r)}{\partial h_{t}}\right|_{r=r_{0}}
$$

To estimate the derivative of interest, $D^{1}$ and $D^{2}(r)$, from equations (10) - (11), $b_{1}$ and $B(r)$ must be estimated. To do so, I estimate the derivative of interest,

$$
D^{1} \equiv \frac{\partial \int_{D_{r}} r f_{t}(r) d r}{\partial h_{t}}=\left(\lambda_{2}-\lambda_{1}\right) \frac{\partial \int_{D_{s}} s f_{t}(s) d s}{\partial h_{t}} \equiv\left(\lambda_{2}-\lambda_{1}\right) D^{s}
$$

in which $\hat{D}^{s}$ can be estimated by

$$
\begin{aligned}
\int_{D_{s}} s f_{t}(s) d s= & b_{0}^{s}+b_{1}^{s} h_{t}+\sum_{i=2}^{p} b_{i}^{s} \int_{D_{s}} s^{i} f_{t}(s) d s+\sum_{j=1}^{q}\left(b_{p+2 j-1}^{s}, b_{p+2 j}^{s}\right) \int_{D_{s}} \phi_{j}(s) f_{t}(s) d s \\
& +\left(u_{t}^{s}-e_{p q t}^{s}\right)
\end{aligned}
$$

where $s$ is normalized temperature, which is defined as $\frac{r-\lambda_{1}}{\lambda_{2}-\lambda_{1}}$ and bounded in unit interval (i.e., $\left.D_{s}=[0,1]\right)$. Define

$$
\begin{aligned}
\beta_{p q}^{s} & =\left(b_{2}^{s}, b_{3}^{s}, \ldots, b_{p+2 q-1}^{s}, b_{p+2 q}^{s}\right)^{\prime} \\
\pi_{p q}(s) & =\left(s^{2}, s^{3}, \ldots, s^{p}, \cos (2 \pi s), \sin (2 \pi s), \ldots, \cos (2 q \pi s), \sin (2 q \pi s)\right)^{\prime}
\end{aligned}
$$


then, we have

$$
\hat{B}^{N}(s)=\hat{B}\left(\frac{r-\lambda_{1}}{\lambda_{2}-\lambda_{1}}\right)=\hat{\beta}_{p q}^{s^{\prime}} \pi_{p q}(s)
$$

due to the normalization-invariance property. Using the estimator $\hat{B}^{N}(s)=\hat{\beta}_{p q}^{s^{\prime}} \pi_{p q}(s)$, we have

$$
\int_{D_{r}} \hat{B}(r) f_{t}(r) d r=\int_{D_{s}} \hat{B}^{N}(s) f_{t}^{N}(s) d s
$$

Consequently, we estimate

$$
\frac{\partial \int_{D_{r}} \hat{B}(r) f_{t}(r) d r}{\partial h_{t}}
$$

by implementing a linear regression of $\int_{D_{s}} \hat{B}^{N}(s) f_{t}^{N}(s) d s$ on $h_{t}$ with a constant.

\subsection{Estimation and Inference}

As is well known, LS method on cointegration analysis provides super-consistent estimator. However, LS estimators may be inefficient and asymptotically biased. Moreover, the hypothesis testing based on LS estimator is invalid, due to the presence of nuisance parameters. In this paper, I employ the canonical cointegrating regression developed by Park (1992).

Equation (12) is simply expressed as

$$
y_{t}=\alpha_{p q}^{\prime} z_{p q t}+u_{p q t}
$$


where

$$
\begin{aligned}
y_{t} & =\int_{D_{s}} s f_{t}(s) d s \\
z_{p q t} & =\left(h_{t}, \int_{D_{s}} s^{2} f_{t}(s) d s, \ldots, \int_{D_{s}} \cos (2 q \pi s) f_{t}(s) d s, \int_{D_{s}} \sin (2 q \pi s) f_{t}(s) d s\right)^{\prime} \\
u_{p q t} & =e_{p q t}+u_{t}
\end{aligned}
$$

with assuming both $y_{t}$ and $z_{p q t}$ are mean zero processes by taking demeaning procedure from equation (14). Estimation results indicate that there is high collinearity between the regressors, which implies that the long-run variance estimate of the firstdifferenced regressors, $\Delta z_{p q t}$, suffers from singularity problem (i.e., the assumption, $\Omega>0$ does not hold, where $\Omega$ is long-run variance of $\left.\left(\Delta z_{p q t}\right)\right)$. In this case, the high collinearity (or cointegrated regressor) prevents estimates of interest from identification. Specifically, estimating equation (12) with ignoring high collinearity results that the mean effect is vanished and estimation errors rapidly decrease as $p$ and $q$ increase, and therefore Wald statistics rapidly increase. In this case, we may not identify the nonlinear TRF effect on global mean temperature anomaly.

In order that the nonlinear part of the left-hand side of equation (6) is independent of linear part of that equation, I follow the way of orthogonalization scheme, derived by Miller (2017), from equation (12), as given by

$$
\begin{aligned}
\int_{D_{s}} s f_{t}(s) d s & =b_{0}+b_{1} h_{t}+\sum_{i=2}^{p} b_{i}\left(\int_{D_{s}} s^{i} f_{t}(s) d s\right)^{*} \\
& +\sum_{j=1}^{q}\left(b_{p+2 j-1}, b_{p+2 j}\right)\left(\int_{D_{s}} \phi_{j}(s) f_{t}(s) d s\right)^{*}+\left(u_{t}-e_{p q t}\right)
\end{aligned}
$$


where

$$
\begin{aligned}
\left(\int_{D_{s}} s^{2} f_{t}(s) d s\right)^{*} & =\int_{D_{s}} s^{2} f_{t}(s) d s-\delta_{21} \int s f_{t}(s) d s-\delta_{20} \\
\left(\int_{D_{s}} \cos 2 \pi s f_{t}(s) d s\right)^{*} & =\int_{D_{s}} \cos 2 \pi s f_{t}(s) d s-\delta_{32} \int s^{2} f_{t}(s) d s-\delta_{31} \int s f_{t}(s) d s-\delta_{30} \\
\left(\int_{D_{s}} \sin 2 \pi s f_{t}(s) d s\right)^{*} & =\int_{D_{s}} \sin 2 \pi s f_{t}(s) d s-\delta_{43} \int \cos 2 \pi s f_{t}(s) d s-\delta_{42} \int s^{2} f_{t}(s) d s \\
& -\delta_{41} \int s f_{t}(s) d s-\delta_{40} \\
& \vdots
\end{aligned}
$$

Consequently, we have orthogonalized response function as

$$
\begin{aligned}
\hat{B}^{*}(s)=\hat{\beta}_{p q}^{*} \pi_{p q}^{*}(s) & =\hat{b}_{2}^{*}\left(s^{2}-\delta_{21} s-\delta_{20}\right)+\hat{b}_{3}^{*}\left(\cos 2 \pi s-\delta_{32} s^{2}-\delta_{31} s-\delta_{30}\right) \\
& +\hat{b}_{4}^{*}\left(\sin 2 \pi s-\delta_{43} \cos 2 \pi s-\delta_{42} s^{2}-\delta_{41} s-\delta_{40}\right)+\ldots
\end{aligned}
$$

To implement the orthogonalization scheme, I employ the least squares method to extract the orthogonal deviations from nonlinear temperature terms. Consequently, I estimate equation (15), instead of estimating equation (12), as simply given by

$$
y_{t}=\gamma h_{t}+\alpha_{p q}^{\prime} \tilde{z}_{p q t}+u_{p q t}
$$

where

$$
\begin{aligned}
y_{t} & =\int_{D_{s}} s f_{t}(s) d s \\
\tilde{z}_{p q t} & =\left(\left(\int_{D_{s}} s^{2} f_{t}(s) d s\right)^{*}, \ldots,\left(\int_{D_{s}} \cos (2 q \pi s) f_{t}(s) d s\right)^{*},\left(\int_{D_{s}} \sin (2 q \pi s) f_{t}(s) d s\right)^{*}\right)^{\prime} \\
u_{p q t} & =e_{p q t}+u_{t}
\end{aligned}
$$


Assume $\left\{w_{p q t}\right\}$ defined by

$$
w_{p q t}=\left(u_{p q t}, \Delta h_{t}\right)^{\prime}
$$

satisfies the Invariance Principle (IP). Define $\phi_{p q}(k)=\mathbb{E} w_{p q t} w_{p q, t-k}^{\prime}$ to be the autocovariance function of $\left(w_{p q t}\right)$. The longrun variance matrix $\Omega$ of $\left\{w_{p q t}\right\}$ is then given by

$$
\Omega=\sum_{k=-\infty}^{\infty} \phi(k)
$$

Here and from now on, I suppress the subscript $p q$ at the variance matrix for notational convenience. I also define the contemporaneous variance $\Sigma=\phi(0)$ and the one-sided longrun variance $\Lambda=\sum_{k=1}^{\infty} \phi(k)$, so that $\Omega=\Sigma+\Lambda+\Lambda^{\prime}$. Further, define

$$
\Gamma=\Sigma+\Lambda
$$

which is $(p+2 q+1) \times(p+2 q+1)$ matrix. Consider the partitioned submatrices of $\Omega$ and $\Gamma$ by

$$
\Omega=\left(\begin{array}{cc}
\omega_{11} & \omega_{12} \\
\omega_{21} & \Omega_{22}
\end{array}\right), \quad \Gamma=\left(\begin{array}{cc}
\gamma_{11} & \gamma_{12} \\
\gamma_{21} & \Gamma_{22}
\end{array}\right)=\left[\begin{array}{ll}
\gamma_{1} & \Gamma_{2}
\end{array}\right]
$$

with assuming $\Omega_{22}>0$. The Canonical Cointegrating Regression (CCR) method proposed by Park (1992) is based on the regression

$$
\begin{aligned}
y_{t}^{*} & =\gamma h_{t}^{*}+\alpha_{p q}^{\prime} \tilde{z}_{p q t}+u_{p q t}^{*} \\
& =\theta_{p q}^{\prime} \nu_{p q t}+u_{p q t}^{*}
\end{aligned}
$$


where $\theta_{p q}=\left[\gamma, \alpha_{p q}\right]^{\prime}$ and $\nu_{p q t}=\left[h_{t}^{*}, \tilde{z}_{p q t}\right]$, formulated with $\left\{y_{t}^{*}\right\}$ and $\left\{h_{t}^{*}\right\}$, which are stationary transformations of $\left\{y_{t}\right\}$ and $\left\{h_{t}\right\}$, which are given by

$$
\begin{aligned}
& y_{t}^{*}=y_{t}-\gamma^{\prime} \Gamma_{2}^{\prime} \Sigma^{-1} w_{p q t}-w_{12} \Omega_{22}^{-1} \Delta h_{t} \\
& h_{t}^{*}=h_{t}-\Gamma_{2}^{\prime} \Sigma^{-1} w_{p q t}
\end{aligned}
$$

The CCR estimator $\widehat{\theta}_{p q}^{C C R}$ of $\theta_{p q}$ is the LS estimator of $\theta_{p q}$ in the transformed regression (17). Note that the long-run relationship between $y_{t}$ and $h_{t}$ in equation (16) remains in the relationship between $y_{t}^{*}$ and $h_{t}^{*}$ in equation (17) because stationary transformation does not affect their long-run relationship. In this sense, CCR estimate, $\hat{\gamma}^{C C R}$, must be the same with LS estimate, $\hat{\gamma}^{L S}$. However, the relationship between $y_{t}$ and the augmented nonlinear temperature terms, $\tilde{z}_{p q t}$, is affected by stationary transformation, implying that estimates, $\hat{\alpha}_{p q}$, could be changed by the CCR transformation of equation (18). ${ }^{7}$

For the feasible CCR estimation, Park (1992) and Park et al. (2010) suggest to estimate the long run variance in equation (18) with LS estimator $\hat{\theta}_{p q}^{L S}$ from equation (16). Note that LS standard errors are not efficient under the presence of endogeneity of error term. However, CCR estimators could be problematic if the long-run variance is not consistently estimated due to nonstationarity in errors, inherited from misspecified order $p$ and $q$. Specifically, orthogonalization scheme for nonlinear temperature terms does not guarantee that orthogonal deviation terms, $\tilde{z}_{p q t}$, are covariance stationary processes, implying the possibility that the long-run variance of $w_{p q t}$ is not well-defined. In this case, we cannot say that CCR estimator is better than LS estimator, suggesting that both CCR and LS methods may not provide correct standard errors under misspecified order $p$ and $q$. In Park et al. (2010) paper, moreover, the role of CCR estimator is to fix only linear part of the regression model. However,

\footnotetext{
${ }^{7}$ Although their estimates are slightly changed via CCR transformation, the shape of TRF response function is robust with respect to estimation methodology.
} 
there is no clear literature that the CCR estimator is better than the LS estimator in the nonlinear model, in which this paper is trying to accomplish. In this vein, finding an exact order $p$ and $q$ is a critical issue. Further, Park et al. (2010) assume stationary distributions with bounded common support.

As a practical matter, I assume that the exact orders of $p$ and $q$ are known for the consistent long-run variance estimation with AIC and BIC criteria, so that nonlinearity or nonstationarity in errors is effectively removed. Further, I reasonably treat the nonstationary distribution with bounded common support by restricting time span to its availability. In this vein, there are both $\mathrm{I}(1)$ and $\mathrm{I}(0)$ variables in the regression equation (16) since stochastic trends of nonlinear temperature terms in equation (15) may be cancelled out by orthogonalization scheme. Note that the asymptotic distributions of LS estimators of $\gamma$ and $\alpha_{p q}$ are identical to the case that we implement the regressions of $y_{t}$ on $h_{t}$ and $\tilde{z}_{p q t}$ separately under the assumption that $u_{p q t}$ and $\tilde{z}_{p q t}$ are asymptotically uncorrelated. Now, I consider the consistent kernel estimator for the long-run variance $\Omega$ of $\hat{w}_{p q t}=\left(\hat{u}_{p q t}, \Delta h_{t}\right)^{\prime}$ that is given by

$$
\hat{\Omega}_{n}=\frac{1}{n} \sum_{|i|<l_{n}} \tau\left(\frac{i}{l_{n}}\right) \sum_{t} \hat{w}_{p q t} \hat{w}_{p q, t-i}
$$

with lag window $\tau$ and lag truncation number $l_{n}$. $\left(\hat{u}_{p q t}\right)$ is LS residual from equation (16). Note that I use the parzen window for $\tau$.

To test the cointegration relationship between Earth's surface mean temperature anomalies and total radiative forcing, I employ the variable addition test (VAT), developed by Park (1990). To do so, I consider the commonly used instrument that added to the regression equation (17) as

$$
y_{t}^{*}=\nu_{p q t}^{\prime} \theta_{p q}+s_{t}^{\prime} \lambda+u_{p q t}^{*},
$$


where $s_{t}=\left(t^{2}, t^{3}\right)$ is the 2-dimensional superfluous regressors, quadratic and cubic trends. The null hypothesis $H_{0}: \lambda=0$ cannot be rejected if there is cointegration between variables in equation (17) in the sense that the added superfluous regressors are irrelevant variables. Under the alternative of spurious regression, superfluous regressors would be significant in the sense that nonstationary errors has some trends. Therefore, the null hypothesis would be rejected if there is no cointegration in equation (17) (i.e., spurious regression). Note that the main strength of the VAT test is easy to implement in addition to robustness of many possible specification errors. Moreover, VAT test prevents the nonlinear cointegration model from including overmany nonlinear temperature terms.

The test statistics VAT is given by

$$
\mathrm{VAT}=\frac{R S S-R S S^{k}}{\hat{\omega}_{n k}^{* 2}} \longrightarrow^{d} \chi_{k}^{2} \quad \text { as } \quad n \rightarrow \infty
$$

where RSS and $\mathrm{RSS}^{k}$ are the sum of squared residuals from equation (17) and equation (19), respectively, and $\hat{\omega}_{n k}^{* 2}$ is the consistent longrun variance estimate ${ }^{8}$ for the CCR errors from equation (19). Note that the VAT statistics is cointegration test for the linear cointegrating regression model and it is feasible in my model because the nonlinear cointegration model can be reduced to linear model in equation (19).

Further, I implement the model specification test using Wald test based on CCR estimator. Note that Wald statistics follows asymptotically chi-square distribution because the limiting distribution of the CCR estimator follows mixed-normal. To do so, I consider the mean-level regression (i.e., $p=1$ and $q=0$ ) as a restricted model. For an unrestricted model, I consider the CCR regression model at optimal $p$ and $q$ order, which is chosen by AIC and BIC criteria. Then, the Wald statistics based on

\footnotetext{
${ }^{8}$ For the lag length selection, I use Andrew's automatic bandwidth selection with the parzen window.
} 
CCR estimator is

$$
W=\frac{R S S^{1}-R S S^{2}}{\hat{\omega}_{n k}^{* 2}} \longrightarrow^{d} \chi_{p+2 q-1}^{2} \quad \text { as } \quad n \rightarrow \infty
$$

where $R S S^{1}$ and $R S S^{2}$ are the sum of squared residuals from a restricted model and a minimum IC-based model, respectively, and $\hat{\omega}_{n k}^{* 2}$ is the consistent longrun variance estimate for the CCR errors from a minimum IC-based model. Notably, rejection of Wald test indicates the statistical significance of the specification error.

\section{Data}

In this paper, the following data sources are employed for the Global/NH/SH temperature anomalies and the total radiative forcing variable. For the Global/NH/SH temperature anomaly data, I employ the Goddard Institute for Space Studies (GISS) surface temperature analysis data from 1880 to 2015, ${ }^{9}$ instead of HadCRUT4 dataset. Basically, GISS and HadCRUT4 datasets are generated from same raw dataset. However, their treatments on the same raw dataset are different. Specifically, the GISS dataset uses an interpolated sea-surface temperature analysis, which fills in the empty grid boxes in the sea-surface temperature data, while HadCRUT4 does not attempt to calculate the values for empty grid boxes. In this regard, HadCRUT4 understates the effect of Arctic temperature anomalies, where the warming has been very large during the past decade. ${ }^{10}$ Moreover, GISS dataset is constructed with $2^{\circ}$ latitude by $2^{\circ}$ longitude grid boxes at monthly frequency, which is higher spatial resolution than HadCRUT4 dataset. The data from higher spatial resolution could generate less errors in estimating cross-sectional distributions of temperature anomalies.

Since the temperatures in land station are measured at different elevation and dif-

\footnotetext{
${ }^{9}$ Downloaded from http://data.giss.nasa.gov/gistemp/references.html, Monthly Anomaly: 250km smoothed on January 30th, 2018.

${ }^{10} \mathrm{http}: / /$ www.metoffice.gov.uk/hadobs/indicators/index.html
} 

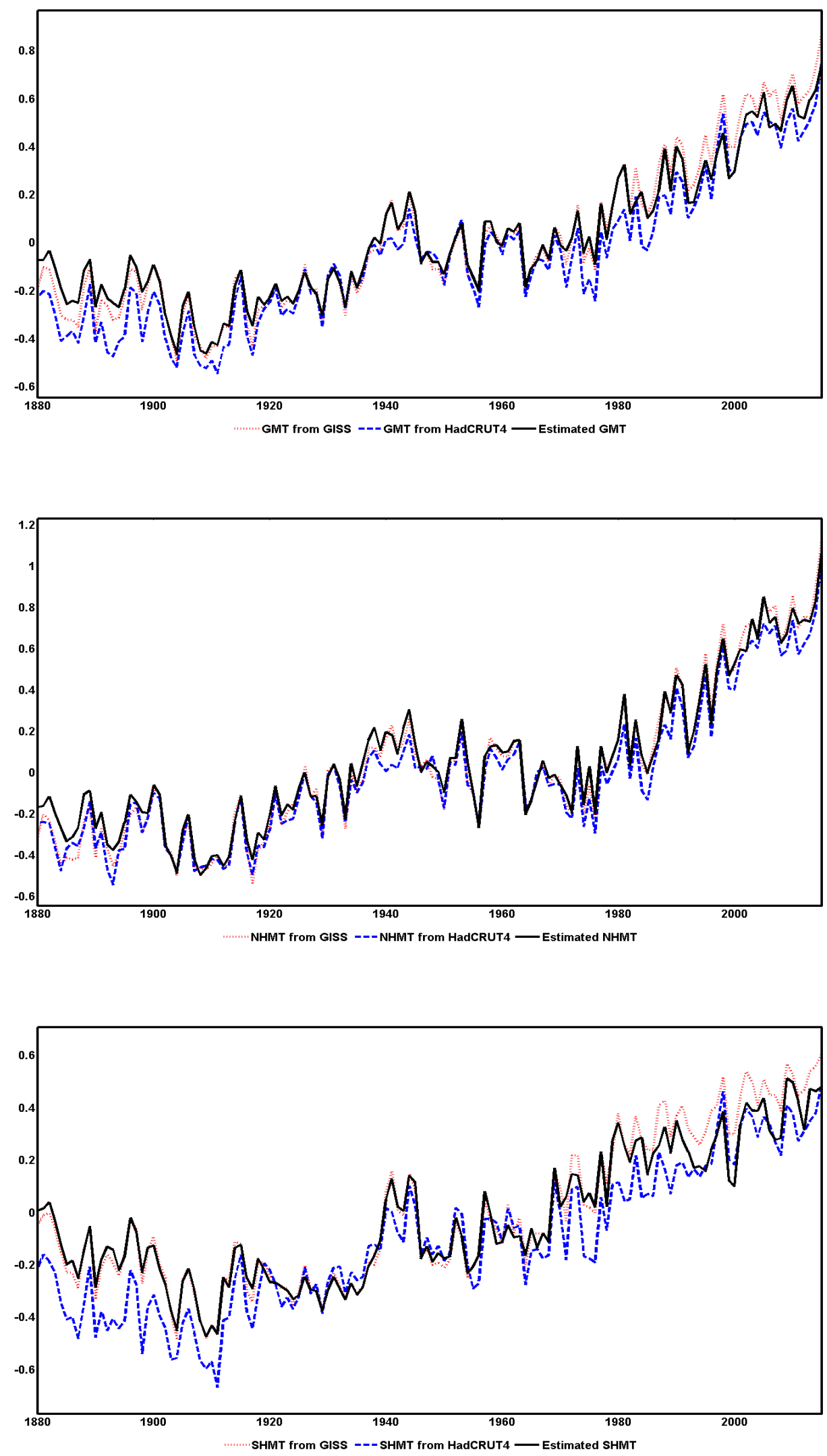

Figure 1: Comparisons of mean temperature anomalies for Globe/NH/SH 


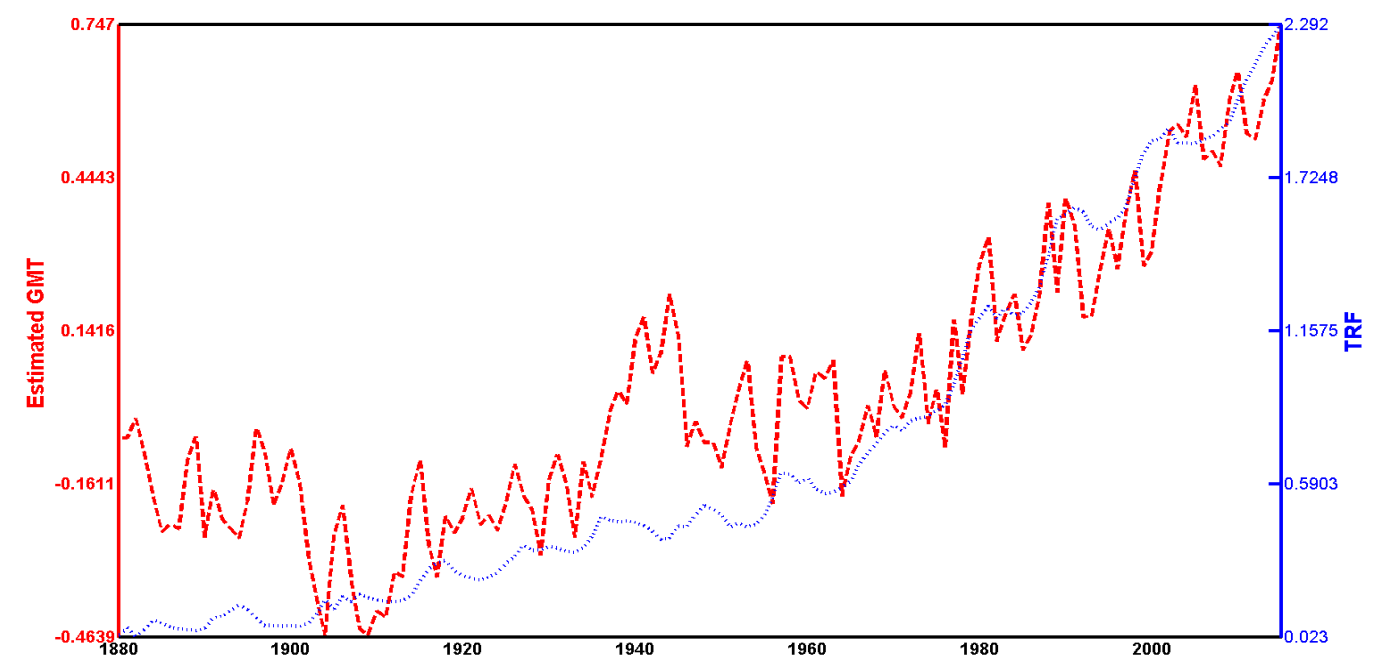

Figure 2: Estimated GMT versus TRF

ferent countries employ different methods, the Global/Northern Hemisphere/Southern Hemisphere temperature data are expressed as anomalies in degrees Celsius from the monthly temperature average from 1951 to 1980, which is the period considered as the "zero-base" (so called climatological normal temperature). Note that since the number of stations and the method of temperature measurement are different across grid boxes, calculating deviation from zero-base may eliminate the heterogeneity across grid boxes over the entire space of the Earth. In this context, I employ the temperature anomaly data from each grid box instead of recovering actual temperature dataset. Further, I exploit the $99 \%$ of the total probability mass as the support for temperature anomaly distributions over the whole time span because I believe that $0.5 \%$ of probability mass at each end is an adequate threshold to minimize the estimation errors induced by boundary problem from standard kernel density estimation technique as well as to provide reasonable descriptive statistics from temperature anomaly distributions, especially first-order moments. The chosen supports for Globe/NH/SH are $[-5.10,5.62],[-6.08,6.58]$, and $[-3.80,3.96]$, respectively.

For the radiative forcing variable, I employ the so-called total radiative forcing 
(TRF) data from 1880 to 2015 (Hansen et al., 2017), which represents the sum of anthropogenic forcing and natural variability. Specifically, the TRF is the the sum of well-mixed greenhouse gases $\left(\mathrm{CO}_{2}, \mathrm{CH}_{4}, \mathrm{~N}_{2} \mathrm{O}\right.$, and CFCs), ozone, surface albedo and tropospheric aerosols, and solar irradiance. ${ }^{11}$ Figure 1 shows calculated mean temperature anomalies from the distributions of temperature anomalies for Globe/NH/SH using GISS dataset, which are compared with web-posted mean temperature anomalies from GISS ${ }^{12}$ and HadCRUT4 (the median of the 100 ensemble member time series) ${ }^{13}$ for Globe/NH/SH. Clearly, Figure 1 shows that $99 \%$ probability mass in estimating temperature anomaly distributions provides a good approximation for estimating first moments (i.e., mean temperature anomalies), in the sense that estimated mean temperature anomalies are very similar with widely-used mean temperature anomalies by the climate scientist. Figure 2 compares calculated mean temperature anomalies with total radiative forcing variable, which illustrates that mean temperature anomaly for Globe clearly move together with the total radiative forcing variable for the last 135 years.

\section{Empirical Analysis}

In this section, I present the estimation results and statistically show that there are nonlinear TRF effects on mean temperature anomalies for Globe/Southern Hemisphere. Throughout this paper, I follow the statistical testing results about the unitroot type nonstationarity from Chang et al. (2016b), which justifies the cointegration analysis. As stated in Chang et al. (2016b), the persistence of global mean temperature anomaly may be closer to a stochastic trend, but not enough to a deterministic trend. Consequently, the failure of rejection of cointegration test implies that total radiative forcing variable shares a stochastic trend with global temperature anomaly

\footnotetext{
${ }^{11}$ Downloaded from www.columbia.edu/ mhs119/Burden on January 30th, 2018.

${ }^{12}$ http://data.giss.nasa.gov/gistemp/

${ }^{13}$ http://www.metoffice.gov.uk/hadobs/hadcrut4/
} 
distribution.

Table 1 shows the estimation results of equation (12) with a derivative of interest. Note that the benefit from orthogonalization scheme is to extract the pure nonlinearity or marginal information from the nonlinear temperature terms, in addition to the stable linear effect of TRF (see Miller, 2017 for details). Based on AIC and BIC criteria, the optimal models for Globe/NH/SH are all $(p=2, q=0) .{ }^{14}$ Moreover, VAT test statistics for Globe/NH/SH and for linear/optimally chosen nonlinear models indicate that all models I consider are authentic that supports the cointegration techniques using CCR methodology. In the literature, the climate sensitivity for globe is estimated by $0.43^{\circ} \mathrm{C} /\left(\mathrm{W} / m^{2}\right)$ and $0.35^{\circ} \mathrm{C} /\left(\mathrm{W} / \mathrm{m}^{2}\right)$ with AMO-unfiltered HadCRUT4 and NASA dataset, respectively (Estrada et al., 2013). ${ }^{15}$ Not surprisingly, the estimate for the climate sensitivity under my linear model is $0.38^{\circ} \mathrm{C} /\left(\mathrm{W} / \mathrm{m}^{2}\right)$, which is a similar value for Globe.

Climatologically, the estimator of the TRF response function at a temperature

Table 1: Estimation results

\begin{tabular}{rrrrrrrrrr}
\hline \hline \multicolumn{8}{c}{ Global Temperature Anomaly } \\
\hline $\mathrm{p}$ & $\mathrm{q}$ & $D^{1}$ & Error(r) & $b_{1}^{C C R}$ & T-stat & AIC & BIC & VAT stat & WALD stat \\
\hline 1 & 0 & 0.378 & -0.046 & 0.035 & 12.429 & -849.451 & -843.625 & 0.444 & 0.000 \\
2 & 0 & 0.424 & 0.000 & 0.038 & 18.050 & -910.920 & -902.182 & 1.896 & 31.014 \\
\hline \multicolumn{7}{c}{ Northern Hemisphere Temperature Anomaly } \\
\hline $\mathrm{p}$ & $\mathrm{q}$ & $D^{1}$ & \multicolumn{1}{c}{ Error(r) } & $b_{1}^{C C R}$ & T-stat & AIC & BIC & VAT stat & WALD stat \\
\hline 1 & 0 & 0.474 & -0.019 & 0.037 & 10.238 & -801.433 & -795.607 & 0.130 & 0.000 \\
2 & 0 & 0.493 & 0.000 & 0.039 & 10.432 & -815.949 & -807.211 & 0.010 & 3.649 \\
\hline \multicolumn{8}{c}{ Southern Hemisphere Temperature Anomaly } \\
\hline $\mathrm{p}$ & $\mathrm{q}$ & $D^{1}$ & Error(r) & $b_{1}^{C C R}$ & T-stat & AIC & BIC & VAT stat & WALD stat \\
\hline 1 & 0 & 0.287 & -0.063 & 0.037 & 8.582 & -749.153 & -743.328 & 0.863 & 0.000 \\
2 & 0 & 0.350 & 0.000 & 0.041 & 10.969 & -798.575 & -789.837 & 1.525 & 14.603 \\
\hline \hline
\end{tabular}

anomaly $r_{0}, D^{2}\left(r_{0}\right)$ indicates that the change in global mean temperature anomaly, if the net incoming absorbed radiation is solely determined by a temperature anomaly, $r_{0}$. As such, the nonlinear effect, in addition to mean effect (or linear effect), could provide the temperature-dependent TRF effect on mean temperature anomalies. On the contrary, the estimator $D^{1}$ represents the climate sensitivity that takes into ac-

\footnotetext{
${ }^{14} \mathrm{AIC}$ criteria gives $\mathrm{p}=1, \mathrm{q}=3$ model only for global case. However, the shape of TRF response function is robust.

${ }^{15}$ With AMO-filtered HadCRUT4 (version 4.2.0.0) and NASA dataset, the climate sensitivity is estimated by $0.40^{\circ} \mathrm{C} /\left(\mathrm{W} / \mathrm{m}^{2}\right)$ and $0.39^{\circ} \mathrm{C} /\left(\mathrm{W} / \mathrm{m}^{2}\right)$, respectively.
} 
count of all nonlinear effects across the Globe, showing that the misspecification error of the linear model is greatest for Southern Hemisphere $\left(-0.063^{\circ} \mathrm{C} /\left(\mathrm{W} / \mathrm{m}^{2}\right)\right)$ and least for Northern Hemisphere $\left(-0.019^{\circ} \mathrm{C} /\left(\mathrm{W} / \mathrm{m}^{2}\right)\right)$. Moreover, Wald test decisively rejects the null of no statistical significance of nonlinear temperature terms for Globe and Southern Hemisphere cases ( $p$-values are less than 0.0001). However, $p$-value of Wald statistics for Northern Hemisphere is 0.056, supporting the linear model at 5\% significance level. Put simply, the climate sensitivities that amount to the nonlinear effect for Globe and Southern Hemisphere are $0.046^{\circ} \mathrm{C} /\left(\mathrm{W} / \mathrm{m}^{2}\right)$ and $0.063^{\circ} \mathrm{C} /\left(\mathrm{W} / \mathrm{m}^{2}\right)$, respectively, and the nonlinear cointegration model may not be necessary for Northern Hemisphere case.

Note that the climate sensitivity of the nonlinear cointegration model for Globe is estimated as a value, 0.424 , indicating that the global mean temperature anomaly increases by $0.424^{\circ} \mathrm{C}$ when the total radiative forcing increases by $1 \mathrm{~W} / m^{2}$. However, the transient climate response is more informative concept than the climate sensitivity, and it is defined as the global mean temperature response in ${ }^{\circ} \mathrm{C}$ to a doubling of atmospheric $\mathrm{CO}_{2}$ from pre-industrial level by an increase of $1 \%$ per year. Schwartz (2012) suggests the TRF level, $3.71 \mathrm{~W} / \mathrm{m}^{2}$ when the atmospheric $\mathrm{CO}_{2}$ is doubled from pre-industrial level, and therefore the estimated transient climate response is $1.573^{\circ} \mathrm{C}\left(=3.71 \times 0.424^{\circ} \mathrm{C} /\left(\mathrm{W} / m^{2}\right)\right)$, which is $0.171^{\circ} \mathrm{C}$ higher than the estimated transient climate response from the linear model $\left(1.402^{\circ} \mathrm{C}\right)$. Given the global mean temperature anomaly was $-0.23^{\circ} \mathrm{C}$ in 1880 , the predicted global mean temperature anomaly associated with a doubled atmospheric $\mathrm{CO}_{2}$ level is $1.343^{\circ} \mathrm{C}$. Note that the global mean temperature anomaly was $0.76^{\circ} \mathrm{C}$ in 2015 , and the TRF level was 2.3 $\mathrm{W} / m^{2}$ in 2015 .

In Figure 3, both the net incoming absorbed radiation $B(r)$ and the change in temperature anomaly distribution associated with TRF change $d_{T}(r)$ are presented for Globe/Southern Hemisphere cases. The dotted line is $95 \%$ bootstrapping confi- 
dence bands. Note that I truncate the domain of temperature anomaly below $1.5^{\circ} \mathrm{C}$ and above $2.5^{\circ} \mathrm{C}$ for a practical reason. The net incoming absorbed radiation term $B(r)$ illustrates that the nonlinear effect becomes stronger when temperature anomaly is at extreme end for both cases. Moreover, a positive (negative) value of $B(r)$ implies that the TRF effect under linear model is underestimated (overestimated). By including nonlinear temperature terms, together with the change in distribution associated with TRF change as a weight, $B(r) d_{T}(r)$, the nonlinear TRF effect is obtained by amplifying or attenuating the linear TRF effect, which is expressed in equation (11). Note that since the change in probability with respect to the change in TRF (i.e., $\left.d_{T}(r)\right)$ at extreme temperature anomaly is very small, the nonlinear effect at extreme temperature anomaly could be much less than that at low or high temperature anomaly. Further note that the linear model is correctly specified at the temperature anomaly where the net incoming absorbed radiation is zero (i.e., $\left\{r \in D_{r} \mid B(r)=0\right\}$ ). At such anomalies, there must be no uncertainty in the nonlinear temperature terms.

Figure 4 illustrates the TRF response function to mean temperature anomalies, which is the estimator, $D^{2}(r)$ for $r \in D_{r}$ in equation (11), for Globe/Southern Hemisphere. The reference (dashed blue) line represents the linear TRF response function, which shows the effect of TRF on mean temperature anomaly without nonlinear effect. This implies that the linear model postulates that the effect of TRF on mean temperature anomaly is constant across temperature anomalies. In addition to linear TRF effect, the nonlinear temperature terms provide temperature-varying TRF effects on mean temperature anomaly over the compact support. In doing so, there are mainly four temperature anomaly zones in the support; extremely low temperature anomaly, low temperature anomaly, high temperature anomaly, and extremely high temperature anomaly zones.

For Globe, the linear model estimates a constant climate sensitivity as a value, $0.40^{\circ} \mathrm{C} /\left(\mathrm{W} / \mathrm{m}^{2}\right)$. Moreover, the nonlinear TRF response function intersects with the 
linear TRF response function at three anomalies, $-0.82,0.12$, and $1.73^{\circ} \mathrm{C}$. For Southern Hemisphere case, on the other hand, the linear TRF response function estimates a constant climate sensitivity as a value, $0.32^{\circ} \mathrm{C} /\left(\mathrm{W} / \mathrm{m}^{2}\right)$, and the intersected anomalies are $-0.47,0.08$, and $2^{\circ} \mathrm{C}$. As such, the nonlinear cointegration model shows that the estimated constant climate sensitivity is underestimated (overestimated) at high (low) temperature anomaly zone. More interestingly, the TRF response function provides an interesting insight that the TRF effect becomes stronger as the temperature anomaly increases from $-0.26^{\circ} \mathrm{C}$ to $0.58^{\circ} \mathrm{C}$, implying that the TRF effect has been increased as the global mean temperature anomaly increases. This also implies that the global warming has been accelerated over the past decades.

In particular, the greatest temperature-dependent TRF effect on global mean temperature anomaly is estimated as a value, $0.51^{\circ} \mathrm{C} /\left(\mathrm{W} / \mathrm{m}^{2}\right)$, if the net incoming absorbed radiation is solely determined by a temperature anomaly, $0.56^{\circ} \mathrm{C}$. Note that the global mean temperature anomaly was $0.58^{\circ} \mathrm{C}$ in 2014 , implying that the effect of TRF on the global mean temperature anomaly was strongest in 2014. It is worth noting that the nonlinear TRF effect for Southern Hemisphere case shows a similar pattern with that for Global case. However, its effect has been smaller than that for Global case, which is reasonable, in the sense that the global warming speed in Northern Hemisphere has been faster than Southern Hemisphere.

\section{Concluding Remarks}

In this paper, I propose the nonlinear cointegration model based on well-known energy balance climate model. Specifically, I investigate the nonlinear cointegrating regression of mean temperature anomalies for Globe/NH/SH on total radiative forcing variable using estimated spatial temperature anomaly distributions. Using distribu- 

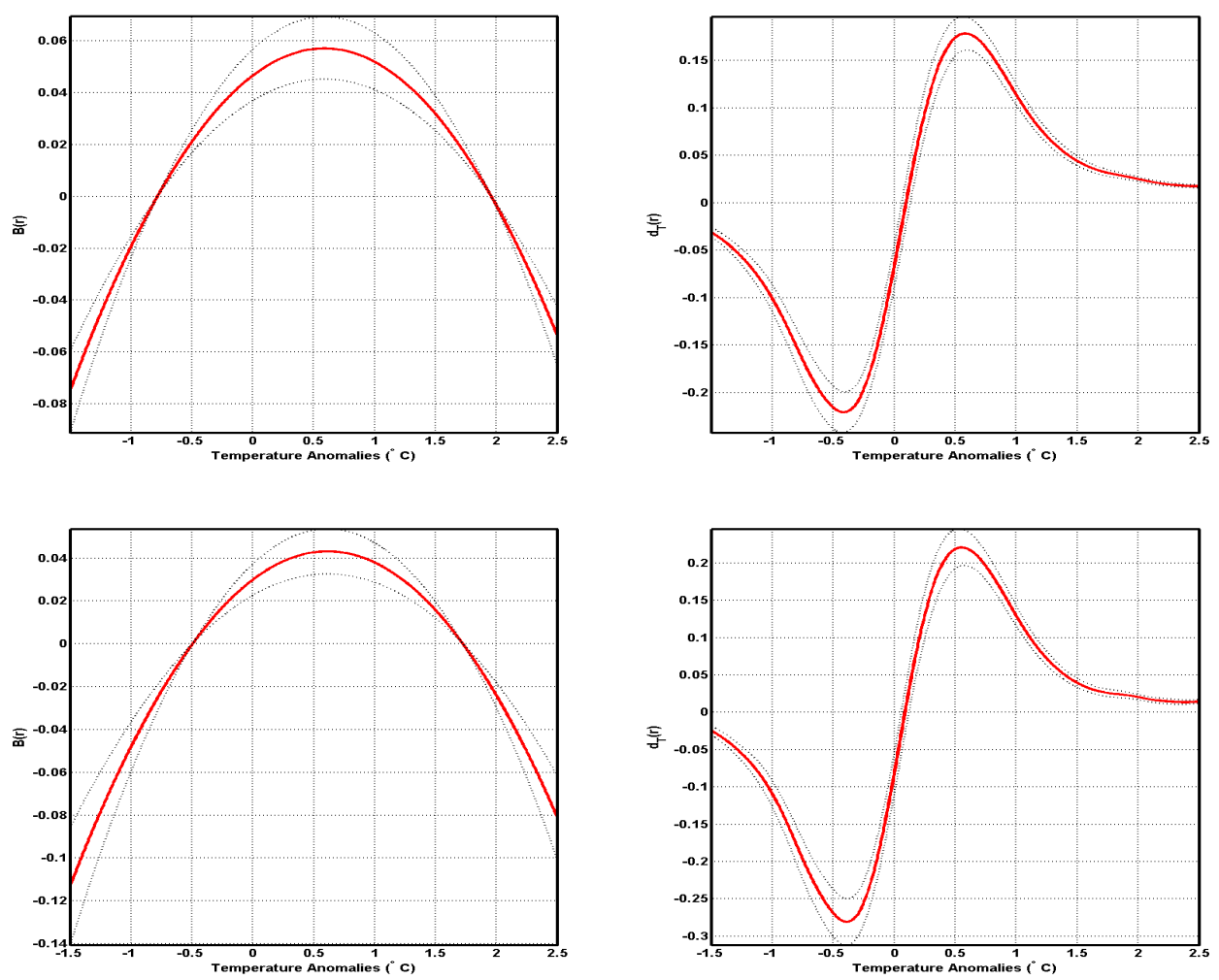

Figure 3: Nonlinear response function $B(r)$ and $d_{T}(r)$ for Globe/Southern Hemisphere. 


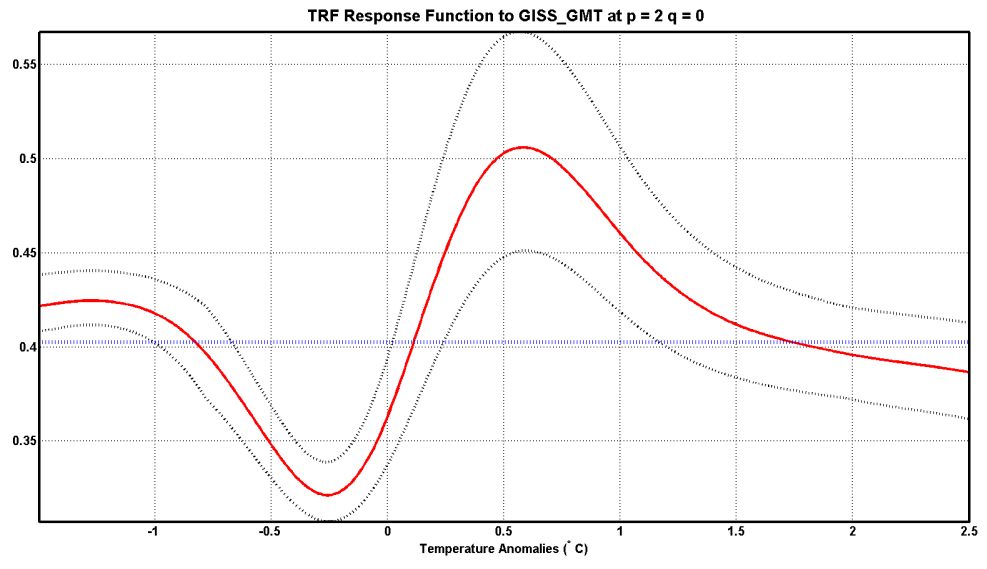

TRF Response Function to GISS_SHMT at $p=2 q=0$

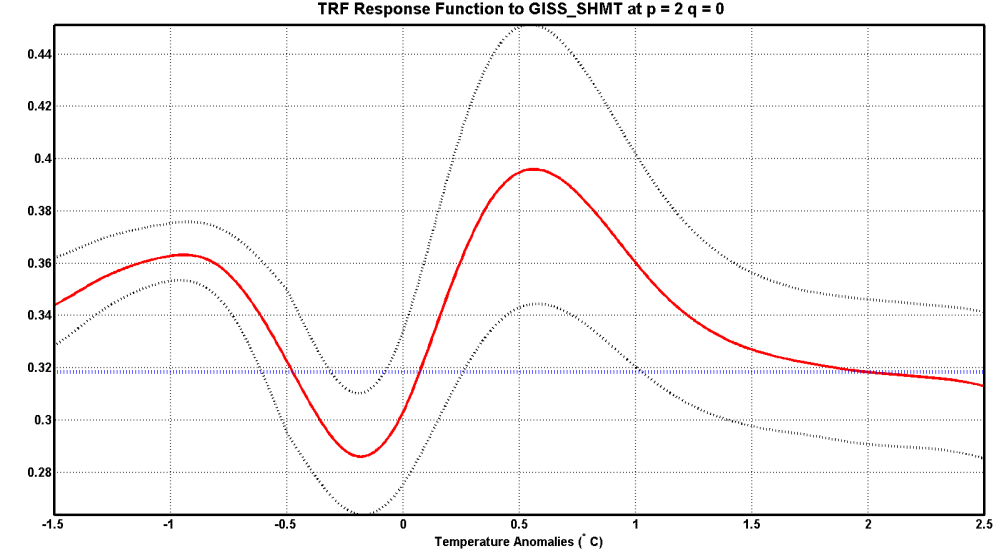

Figure 4: TRF response function to mean temperature anomalies for Globe/Southern Hemisphere (see equation (11)). 
tional information of the temperature anomaly data, I estimate the nonlinear TRF effect on mean temperature anomalies, suggesting that the TRF effects on mean temperature anomalies are temperature-dependent for Globe and Southern Hemisphere cases. Graphically, the TRF response function has a flexible shape to represent the change in mean temperature anomalies when the net incoming absorbed radiation is hypothetically determined at some temperature anomalies.

Statistically, the linear model fails to take account of a net incoming absorbed radiation term, which invalidates a slope estimator. Considering the functional form of the net incoming absorbed radiation provided in the literature, the proposed nonlinear cointegration model shows an acceptable nonlinear dependence structure between mean temperature anomalies and total radiative forcing. Specifically, the climate sensitivity becomes higher (lower) if the temperature that determines net incoming absorbed radiation is a positive (negative) value. With VAT and Wald statistics, I statistically show that the linear model possesses a significant misspecification error for Globe and Southern Hemisphere cases.

The next direction of this research is to take into account climate variability. As Brock et al. (2013) emphasize, the spatial complexity on the Earth is a big challenge in analyzing temperature series at hemispheric scales. The complexity inherited from spatial diversity produces hardly explainable natural variability through the observed data. At least so far, the well-known inter-annual global variability or hemispheric climate variability are the El Niño/Southern Oscillation (ENSO), the North Atlantic Oscillation (NAO) and the Atlantic Multidecadal Oscillation (AMO). Among them, the most influential natural variation on attribution study is the AMO that could distort global warming long-term trend. Specifically, large ocean-atmosphere cycle over the North Atlantic, which is defined as approximately 60 to 90 years low-frequency pattern of sea surface temperature variability, explains larger variability of Northern Hemisphere temperatures and therefore globally. 
In particular, Estrada et al. (2013) point out its difficulty when conducting attribution study. They argue that the detrended Global/Northern Hemisphere temperatures with forcing variables could be further explained by the AMO and therefore the difference in dates of a structural break between them could be explained by the AMO. In this vein, Estrada et al. (2013) filtered AMO information from global/NH mean temperature anomalies to estimate a constant climate sensitivity. To put these factors in perspective, it is worth to estimate the nonlinear climate sensitivity after extracting the major climate variabilities. 


\section{References}

Andrews, D. W. (1991). "Heteroskedasticity and Autocorrelation Consistent Matrix Estimation," Econometrica 59, 817858.

Andrews, D. W. (1991). "Asymptotic Normality of Series Estimators for Nonparametric and Semiparametric Regression Models," Econometrica 59, 307-345.

Bierens, H. J. (2000). "Nonparametric Nonlinear Cotrending Analysis with an Application to Interest and Inflation in the United States," Journal of Business $\mathcal{G}^{3}$ Economic Statistics 18, 323-337.

Bindoff, N. L., Stott, P. A., AchutaRao, K. M., Allen, M. R., Gillett, N., Gutzler, D., Hansingo, K., Hegerl, G., Hu, Y., Jain, S., Mokhov, I. I., Overland, J., Perlwitz, J., Sebbari, R., and Zhang, X. (2013) "Detection and Attribution of Climate Change: from Global to Regional, in: Stocker, T. F., D. Qin, G.-K. Plattner, M. Tignor, S.K. Allen, J. Boschung, A. Nauels, Y. Xia, V. Bex and P.M. Midgley, (Eds.), Climate Change 2013: The Physical Science Basis," Contribution of Working Group I to the Fifth Assessment Report of the Intergovernmental Panel on Climate Change. Cambridge University Press, Cambridge, pp. 867952.

Brock, W.A., G. Engstrom, D. Grass, A. Xepapadeas. (2013). "Energy Balance Climate Models and General Equilibrium Optimal Mitigation Policies," Journal of Economic Dynamics \& Control 37, 2371-2396.

Budyko, M. I. (1969). "The Effect of Solar Radiation Variations on the Climate of the Earth," Tellus 21, 611-619.

Chang, Y., Kim, C., and Park, J. Y., (2016a). "Nonstationarity in Time Series of State Densities," Journal of Econometrics 192, 152-167. 
Chang, Y., Kaufmann, R.K., Kim, C.S., Miller, J.I., Park, J.Y., and Park, S (2016b). "Time Series Analysis of Global Temperature Distributions: Identifying and Estimating Persistent Features in Temperature Anomalies," working paper.

Chang, Y., Kim, C.S., Miller, J.I., Park, J.Y., and Park, S (2014). "Time-varying Long-run Income and Output Elasticities of Electricity Demand with an Application to Korea," Energy Economics 46, 334-347.

Chang, Y., Kim, C.S., Miller, J.I., Park, J.Y., and Park, S (2015). "A New Approach to Modeling the Effects of Temperature Fluctuations on Monthly Electricity Demand," working paper.

Estrada, F. and Perron, P. (2012). "Breaks, Trends and the Attribution of Climate Change: A Time-Series Analysis," Department of Economics, Boston University, mimeo.

Estrada, F. and Perron, P. (2014). "Detection and Attribution of Climate Change Through Econometric Methods," Boletín de la Sociedad Matemática Mexicana 20, 107-136.

Estrada, F., Perron, P., Gay-García, C., and Martínez-López, B. (2013a). "A Time-Series Analysis of the 20th Century Climate Simulations Produced for the IPCCs Fourth Assessment Report," PLoS ONE 8, e60017.

Estrada, F., Perron, P., and Martínez-López, B. (2013b). "Statistically Derived Contributions of Diverse Human Influences to Twentieth-Century Temperature Changes ," Nature Geoscience 6, 10501055.

Gallant, A.R. (1981). "On the Basis in Flexible Functional Forms and an Essentially Unbiased Form: the Fourier Flexible Form," Journal of Econometrics 15, 211245. 
Gay-Garcia, C., Estrada, F., and Sánchez, A. (2009). "Global and Hemispheric Temperatures Revisited," Climatic Change 94, 333-349.

GISTEMP Team, 2016: GISS Surface Temperature Analysis (GISTEMP). NASA Goddard Institute for Space Studies. Dataset accessed 2016-08-06 at http://data.giss.nasa.gov/gistemp/.

Hansen, J., Ruedy, R., Sato, M., and Lo, K. (2010). "Global Surface Temperature Change," Reviews of Geophysics 48, RG4004, doi:10.1029/2010RG000345.

Hansen, J., and Sato, M. (2012). "Paleoclimate Implications for Human-made Climate Change," Climate Change: Inferences from Paleoclimate and Regional Aspects A. Berger, F. Mesinger, and D. Śijaćki, Eds. Springer, 21-48, doi:10.1007/978-3-7091-0973-1_2.

Hansen, J., Sato, M., Kharecha, P., and von Schuckmann, K. (2011). "Earth's Energy Imbalance and Implications," Atmos. Chem. Phys., 11, 13421-13449, doi:10.5194/acp-11-13421-2011.

Hansen, J., Sato, M., Kharecha, P., von Schuckmann, K., Beerling, D.J., Cao, J., Marcott, S., Masson-Delmotte, V., Prather, M.J., Rohling, E.J., Shakun, J., Smith, P., Lacis, A., Russell, G., and Ruedy, R. (2017). "Young People's Burden: Requirement of Negative $\mathrm{CO}_{2}$ Emissions," Earth Syst. Dynam., 8, 577-616, doi:10.5194/esd-8-577-2017.

Hansen, J., Sato, M., Russell, G., and Kharecha, P. (2013). "Climate Sensitivity, Sea Level and Atmospheric Carbon Dioxide," Phil. Trans. Roy. Soc. A, 371, 20120294, doi:10.1098/rsta.2012.0294.

Held, I.M., and Suarez M.J. (1974). "Simple Albedo Feedback Models of the Icecaps," Tellus 36, 613-628. 
Intergovernmental Panel on Climate Change (IPCC, 2007). Climate Change 2007: The Physical Science Basis, Contribution of Working Group I to the Fourth Assessment Report of the Intergovernmental Panel on Climate Change [Core Writing Team, Solomon, S., Qin, D., Manning, M., Chen, Z., Marquis, M., Averyt, K.B., Tignor, M., and Miller, H.L. (eds.)]. Cambridge University Press, ISBN 978-0-521-88009-1 (pb: 978-0-521-70596-7).

Intergovernmental Panel on Climate Change (IPCC, 2014). Climate Change 2014: Synthesis Report. Contribution of Working Groups I, II and III to the Fifth Assessment Report of the Intergovernmental Panel on Climate Change [Core Writing Team, R.K. Pachauri and L.A. Meyer (eds.)]. IPCC, Geneva, Switzerland, $151 \mathrm{pp}$.

Kaufmann, R.K., Kauppi, H., Mann, M.L., and Stock J.H. (2013). "Does Temperature Contain a Stochastic Trend: Linking Statistical Results to Physical Mechanisms," Climatic Change 118, 729-743.

Kaufmann, R.K., Kauppi, H., and Stock, J.H. (2006a). "Emissions, Concentrations and Temperature: A Time Series Analysis," Climatic Change 77, 249-278.

Kaufmann, R.K., Kauppi, H., and Stock, J.H. (2006b). "The Relationship Between Radiative Forcing and Temperature: What Do Statistical Analyses of the Instrumental Temperature Record Measure? Climatic Change 77, 279-289.

Kaufmann, R.K., Kauppi, H., and Stock, J.H. (2010). "Does Temperature Contain a Stochastic Trend? Evaluating Conflicting Statistical Results," Climatic Change 101, 395-405.

Kaufmann, R.K. and Stern, D.I (2002). "Cointegration Analysis of Hemispheric Temperature Relations," Journal of Geophysical Research 107, 10.129/2000JD000174. 
Miller, J.I. (2017). "Decomposing Climate Sensitivity: A Statistical Approach for a Spatially Heterogeneous Planet," University of Missouri, mimeo.

Miller, J.I. and Nam, K (2017). "Dating Hiatuses: A Statistical Model of the Recent Hiatus in Global Warming - and the Next One," University of Missouri, mimeo.

North, G.R. (1975). "Analytical Solution to a Simple Climate Model with Diffusive Heat," Journal of Atmospheric Sciences 32, 1301-1307.

North, G.R., Mengel, J.G., and Short, D.A. (1983). "Simple Energy Balance Model Resolving the Seasons and the Continents: Application to the Astronomical theory of the Ice Ages," Journal of Geophysical Research 88(C11), 65-76.

Newey, W.K. and West, K.D (1987). "A Simple Positive Semi-definite, Heteroskedasticity and Autocorrelation Consistent Covariance Matrix," Econometrica, 55, 703-708.

Park, C. (2010). "How Does Changing Age Distribution Impact Stock Prices? A Nonparametric Approach," Journal of Applied Econometrics 25, 1155-1178.

Park, J.Y. (1990). "Testing for Unit Roots and Cointegration by Variable Addition," in Rhodes, G.F. and T.B. Fomby (ed.), Advances in Econometrics. JAI Press, Greenwich.

Park, J. Y. (1992). "Canonical Cointegrating Regressions," Econometrica, 60, 119143.

Park, J. Y., Shin, K., and Whang, Y (2010). "A Semiparametric Cointegrating Regression: Investigating the Effects of Age Distributions on Consumption and Saving," Journal of Econometrics 157, 165-178.

Petit J. R., (Contributor), (2001). "Vostok Ice Core Data for 420,000 years. IGBP PAGES/World Data Center for Paleoclimatology Data Contribution Series," \# 
2001-076. NOAA/NGDC Paleoclimatology Program, Boulder CO, USA.

Pretis, Felix. (2015). "Econometric Models of Climate Systems: The Equivalence of Two-Component Energy Balance Models and Cointegrated VARs," working paper.

Schwartz, S. E. (2012). "Determination of Earths Transient and Equilibrium Climate Sensitivities from Observations over the Twentieth Century: Strong Dependence on Assumed Forcing," Surveys in Geophysics 33, 745-777.

Stern, N. (2008). "The Economics of Climate Change," American Economic Review, $98(2), 1-37$. 


\section{CHAPTER 2}

\section{Dynamic Factors and Climate Uncertainty in Global Commodity Market}

\section{Introduction}

This PAPER InVESTigAtes how the uncertainty in climate events and dynamic factors affect the global commodity price. The former represents climate uncertainty, and the latter represents the factors that are designed to explain the comovement of the global commodity prices. Despite its growing attention, there has been no clear consensus about climate effects on the global economy. Although global warming, which represents an increasing low-frequency trend in global mean temperature, is believed to have serious detrimental effects on the world economy, its effect is usually emphasized for the future economy. Rather, a vast literature provides the statistical analyses to identify whether the recent warming is anthropogenic or the part of long-term natural variability. The statistical relationships between global mean temperature anomaly and atmospheric $\mathrm{CO}_{2}$ concentration and other forcings are extensively investigated by Kaufmann et al. (2006a, 2006b, 2010, 2013), Estrada et al. (2013), Miller and Nam (2017), and Miller (2017).

Interestingly, the question about how the past and current economies have been affected by the climate fluctuations is still a debated issue. Besides a long-term global warming trend, the interannual climate fluctuations are known to have various effects on the world economy. Among them, the El Niño is one of the most influential climate variability that affects the global climate system with a cycle of four to five years and 
lasting for 9 to 12 months. Specifically, the El Niño is a quasi-periodic warming phase of the central and eastern tropical Pacific ocean (from $170^{\circ} \mathrm{W}$ to $120^{\circ} \mathrm{W}$ longitude and from $5^{\circ} \mathrm{N}$ to $5^{\circ} \mathrm{S}$ latitude, so called Niño 3.4 region), while the La Niña is a quasiperiodic cooling phase in the same area. Since warmer/cooler than normal ocean temperatures are strongly related to the atmospheric pressure oscillation (Bjerknes, 1969), climate scientists broadly refer to these coupled ocean-atmosphere phenomena to the El Niño/Southern Oscillation (ENSO).

The economic causal relationship between the ENSO and the global commodity market is well-established in the literature. Because the ENSO triggers many climate events such as floods, droughts, and hurricanes over the globe, many commodity prices, which are largely produced by directly ENSO-influenced countries, are inevitably affected by the ENSO fluctuations, implying that the effects of the ENSO heavily depend on geographical characteristics. For instance, ENSO-induced droughts and as a result, forest fires in Australia create the negative supply shock to agricultural commodities, generating food price inflation, which leads to higher primary commodity price. On the other hand, ENSO-induced heavier rains and flooding in California and the Southern United States create the positive supply shock to the U.S. particular agricultural industry such as limes, almonds, and avocados, stabilizing commodity price inflation (Cashin et al, 2017).

Surprisingly, the role played by changes in the uncertainty shock has been ignored in the climate economics literature. When a global commodity producer decides on their planting crop, the climate uncertainty has negative effects on crop production. In fact, a company that produces primary commodities such as coffee may postpone or substitute its planting crops if it cannot discern whether a climate fluctuation is a normal event or an extreme weather event, implying the inflationary pressure on the global commodity market. In the meantime, investors may reallocate their commodity portfolio because they want to hedge against climate uncertainty. For instance, 
investors may want to buy more gold in times of climate uncertainty in the sense that the gold is often considered as a safe haven asset or a store of value. Such extreme weather events mainly occurred during the super ENSO period generate unexpected temperature and precipitation changes, and therefore investors' and commodity producers' fear, which leads to adverse effects on the real commodity market.

It is important to note that an uncertainty shock, which will be defined as the effect of the conditional second moment of the ENSO fluctuation, is distinguished from the effect of a first moment shock. Specifically, business decisions are also determined based on the expectations about future abnormal weather event, even though there is no actual change in the current temperature. In this light, if there is an unusual temperature change, which increases the climate uncertainty, the real effect of a level shock is amplified due to the uncertainty effect. Moreover, it is believed that the developed countries such as the United States and Europe are relatively less affected by climate fluctuations than developing countries because abundant resources can reduce climate risks through diversification in a wealthy economy, implying that the uncertainty of the climate event is more influential on the commodities that are produced in developing countries.

Notable works for price uncertainty have been conducted by Elder (2004), Elder and Serletis $(2009,2010)$ and Jo (2014) for U.S., Canada, and global oil markets. As the uncertainty in oil price increases, a firm's optimal investment decision is postponed, resulting in a negative effect of oil price uncertainty on aggregate output (Bernanke, 1983). Moreover, Bloom (2009) shows that a large macro uncertainty shock, defined by the time varying second moment of the driving process, generates a rapid drop-rebound-overshoot dynamics in employment, output, and productivity growth, in the sense that firms delay their hiring and investment in the short-run, and recover their activity after noting their pent-up demand for labor and capital. A similar effect can be expected in global commodity markets with the climate uncer- 
tainty.

This paper relates to the literature that analyzes the effect of ENSO on the global economy. Due to its similarity with the macroeconomic business cycle, identifying the macroeconomic consequences of ENSO effect has been attempted in several papers. Typically, three macroeconomic variables are considered for the target variables: real GDP growth rates, CPI inflation rates, and primary commodity inflation rates. Since it is widely known that there are many covariates to move such macroeconomic variables, identifying the effect of a climate event from those covariates is a difficult issue. With such an identification difficulty, there has been no clear consensus on the climate causality. Brunner (2002) finds the statistically significant effect of the ENSO fluctuation on the increase in real non-oil primary commodity prices using vector autoregressive (VAR) models. Laosuthi and Selover (2007) also discover the statistical significant ENSO effect on commodity price inflation for maize, sorghum, rice, parm oil, and coconut at both quarterly and yearly frequencies.

Moreover, the nonlinearity in the ENSO effect has been documented in several studies. Ubilava $(2012,2014,2017)$ identify the nonlinear relationship between the ENSO and real commodity prices using time-varying smooth transition vector error correction model. Specifically, Ubilava (2012) studies asymmetric ENSO effects on world coffee prices. He concludes that the El Niño shock decreases the price of the Arabica coffee and increases the price of the Robusta coffee, in the sense that Central American countries benefiting from the El Niño mostly produce Arabica coffee and Southeast Asia countries damaged by the El Niño mostly produce Robusta coffee. In addition, Cashin et al. (2017) provide asymmetric ENSO effects on real GDP growth rates using the global VAR models. They conclude that most countries show that the impulse responses from an El Niño shock are greater than those from a La Niña shock with opposite directions. Another type of nonlinear effect of temperature on the economic production has been studied by several authors. In particular, Burke et al. 
(2015) study the nonlinear effect of temperature level on the economic production, whereas Schlenker and Roberts (2009) provide the nonlinear effect of temperature level on the crop yields in the United States.

Throughout this paper, I employ the time-varying factor-augmented VAR with stochastic volatility in mean model to identify the effect of climate uncertainty on the global commodity markets. The factor-augmented VAR (FAVAR), well-established by Bernanke et al. (2005), has become an important tool to identify the policy effect using the information from a large data set. In the meantime, stochastic volatility in mean model is exploited by many researchers to identify the uncertainty effect (Mumtaz and Zanetti, 2013; Jo, 2014; Mumtaz and Theodoridis, 2018). With a one-step Bayesian approach, I utilize a time-varying factor-augmented VAR with stochastic volatility in mean model. In the measurement equation, specifically, I estimate the dynamic factor model with time-varying factor loading to explain the comovement of the global commodity prices. In the transition equation, I estimate the stochastic volatility in mean model to identify the statistical effect of a volatility shock of the ENSO on the dynamic factors extracted from the measurement equation.

The contributions of this paper are threefold. First, I identify the effect of climate uncertainty on the global commodity markets by modifying the existing FAVAR with stochastic volatility in mean model of Mumtaz and Theodoridis (2018) who identify the effect of the common uncertainty on real economic activity and financial variables in the U.S. In addition to identifying the common uncertainty of the global commodity markets, I estimate the climate uncertainty and then identify its effect on the global commodity markets, while the literature mainly focuses on the level effect of a climate event. Second, I extract the covariance-stationary climate factor from the ENSO variable, and then provide the detailed statistical analysis. The ENSO variable, which is known to have two regimes (El Niño and La Niña), has a strong 4-5 year cycle, which makes difficult to use a standard VAR model with the macroeco- 
nomic variables. Using a newly developed endogenous regime switching approach of Chang et al. (2017), I overcome the statistical problem generated by the periodicity in the climate variable.

Third, I identify the commonalities extracted from the global commodity markets via two different classical approaches, and provide more detailed information about omitted macroeconomic factors. It is important to note that the climate economics literature mainly focuses on providing qualitatively interpretable results with a small-scale VAR model. However, the global commodity prices are affected by several economic factors and therefore estimated responses to a climate impact generated from a small-scale VAR model may suffer from the omitted variable bias or model specification problem. In this light, it is necessary to control such macroeconomic effects on global commodity price, in order to identify the climate effect. By exploiting the dynamic factor analysis that summarizes the potentially significant information, this paper aims to fill this gap in the literature.

The rest of the paper is organized as follows. Section 2 presents econometric model and methodology I employ. Section 3 provides the details of the ENSO variable and its uncertainty. Section 4 provides the dynamic factor analyses and identifies the commonalities of the global commodity markets. In Section 5, I present and discuss the empirical results with an application to the world coffee and gold prices, and I conclude with Section 6 .

\section{Econometric Model and Methodology}

Similar to uncertainty of oil price and the U.S. economy defined by Jo (2014) and Jurado et al. (2015), inter alia., climate uncertainty is defined as the conditional standard deviation of the one-period-ahead unforecastable component of the ENSO cycle. To measure the effect of climate uncertainty on the global commodity market, 
two different approaches can be utilized to provide volatility in mean effect: stochastic volatility in mean model via Bayesian approach and non-stochastic volatility in mean model via classical approach. In Elder and Serletis (2009, 2010), conditional on past information, time-varying volatility is deterministically modeled as a generalized autoregressive conditional heteroskedasticity (GARCH) process and is included in the mean equation of the VAR system.

To identify the uncertainty effect, they provide a conventional statistical test on the coefficient of oil price uncertainty in the real GDP equation. Moreover, they compare the impulse response function from the shock that increases the conditional first moment of oil price (with volatility-in-mean effect) with the impulse response function from restricting the coefficient on oil price uncertainty to zero (without volatility-inmean effect). Note that the impulse response function without MGARCH-in-Mean effect may be invalid, if the MGARCH-in-mean effect is significant, but omitted. In this context, one of their contributions is regarded as providing impulse response functions of GDP growth rate to asymmetric shocks on oil price, which take account of the uncertainty effect, rather than analyzing the pure uncertainty effect of oil price fluctuations.

As Jo (2014) emphasizes, the volatility process in GARCH setup is fully determined by the changes in the past innovations or the lagged conditional variances, which is not a realistic assumption. In particular, the time-varying volatility of the sea surface temperature has unpredictable movements conditional on past information of that temperature. To identify the effect of the oil price uncertainty, the shock that increases the conditional second moment of oil price must be separated from the shock that increases the conditional first moment of oil price. In a GARCH setup, however, the shock that increases the conditional first moment of oil price automatically increases volatility, and therefore there is no shock to generate an exogenous movement of the volatility process. To avoid this issue, Jo (2014) includes volatil- 
ity terms, which are driven by innovation terms that provide an independent source of movement to the volatility dynamics (i.e., volatility-specific shocks), in the mean equation of the VAR system. Moreover, in addition to the asymmetric effect of the ENSO, the volatility feedback effect can be tested by the stochastic volatility-in-mean model (see Koopman and Uspensky, 2002, for details). All things considered, it is highly desirable to incorporate stochastic movement conditional on past information into the volatility process.

It is worth noting that the global commodity prices do not only depend on climate fluctuation. Certainly, the global commodity prices have many covariates, and those covariates are likely to be mediators for the ENSO variable, creating an identification problem in estimating the effect of a climate impact. As explained in Section 4, the global commodity markets are largely driven by the changes in global fuel and non-fuel commodity prices. Obviously, those global factors could be significantly influenced by the climate events. Due to data availability and macroeconomic variable selection problem, it is difficult to incorporate such mediators in an explicit way. Moreover, incorporating many mediators in the VAR model suffers from the problem of parameter proliferation. This paper, instead, employs the factor model to circumvent not only estimation of too many parameters, but also an omitted variable issue. Specifically, I incorporate the dynamic factors, which represent the commonality of the whole commodity markets, in my analysis. Note that the comovement in global commodity markets has already been documented in Pindyck and Rotemberg (1990), Chiaie et al. (2017), inter alia. Such estimated commonalities approximate omitted economic factors from the small-scale VAR model. In this light, I utilize the factor augmented VAR model in the spirit of Bernanke et al. (2005).

Typically, the static principal component analysis (PCA) is employed to summarize the information contained in the data by decomposing the total variability into a small number of components. Since it is difficult to explain the extracted prin- 
cipal components economically (Belviso and Milani, 2006), many researchers have extracted the factors of categorized variables with additional restrictions on the structural VARs, namely, structural factors (e.g., real activity or inflation factor). In this case, however, the number of variables of each subset of the original dataset may not be enough to extract the common factors nonparametrically. In addition to the interpretation issue, the number of factors needs to be determined with the tradeoff between better-fit and efficiency (Bai and Ng, 2002).

Throughout this paper, I employ the full Bayesian approach to jointly estimate the measurement and transition equations, and then compare extracted dynamic factors with the principal components extracted by the classical approaches. Note that the classical maximum likelihood estimation of the state space model with kalman filter is known to have a good performance when the number of cross-sectional dimensions, $N$ is small and there is no nonlinearity in the transition equation. When $N$ is large with no nonlinearity in the model, however, the common factors are extracted by the principal component analysis with a two-step approach. This is because the consistency in factor estimates is provided when the number of cross-sectional dimensions $N$ is large relative to the number of temporal dimensions $\bar{T}$, suggesting the two-step approach for computational efficiency. Otherwise, the bootstrapping procedure for uncertainty in factor estimates is necessary or the one-step Bayesian approach has to be considered.

\subsection{Time-varying Factor-Augmented VAR with Stochastic Volatility-in-Mean Model}

A vast empirical literature has investigated the small-scale structural VAR model with volatility-in-mean effect. Based on univariate stochastic volatility-in-mean model, provided in Koopman and Uspensky (2002), the multivariate stochastic volatility-inmean model has been studied by many researchers, including Jurado, Ludvigson, and 
Ng (2015), Asai and McAleer (2009), Mumtaz and Zanetti (2013), Jo (2014), and Shin and Zhong (2018), inter alia. In the meantime, a large empirical literature has investigated the large-scale VAR system such as factor-augmented VAR and Global VAR models. In particular, the factor-augmented VAR model, rigorously developed by Bernanke et al. (2005), is investigated by Mumtaz and Surico (2009), Mumtaz (2010), Liu et al. (2014), and Chang and Kwak (2017), inter alia. Recently, two small and large scale VAR systems are investigated together in Mumtaz and Theodoridis (2018), who provide time-varying impulse responses of real economic activity and financial variables in the U.S. to uncertainty shocks via one-step Bayesian approach.

Throughout this paper, I closely follow the time-varying factor-augmented VAR with stochastic volatility-in-mean model, developed by Mumtaz and Theodoridis (2018). The model can be written with measurement and transition equations. The measurement equation is given by

$$
\begin{aligned}
& X_{i t}=\Lambda_{i t}^{T} T_{t}+\Lambda_{i t}^{F} F_{t}+R_{i}^{\frac{1}{2}} \varepsilon_{i t} \\
& \Lambda_{i t}=\Lambda_{i t-1}+\bar{\eta}_{i t}, \quad \operatorname{var}\left(\bar{\eta}_{i t}\right)=Q_{\Lambda}
\end{aligned}
$$

where $\Lambda_{i t}=\left[\Lambda_{i t}^{T} \Lambda_{i t}^{F}\right], \Lambda_{i t}^{T}$ and $\Lambda_{i t}^{F}$ are $\bar{T} \times 1$ and $\bar{T} \times(N-1)$ matrices of time-varying factor loadings, and $\varepsilon_{i t}$ is a $\bar{T} \times 1$ vector of mean-zero errors. Note that $\bar{T}$ and $N-1$ are the temporal dimension and the number of macroeconomic factors, respectively. To identify the climate effect, the ENSO variable, $T_{t}$ is included in the measurement equation. ${ }^{1}$ The detailed statistical analyses of the climate variable $T_{t}$ and the macroeconomic factors $F_{t}$ are provided in Section 3 and Section 4, respectively. Note that I standardize the dataset $X_{t}$ before conducting analysis so that all detrended log real commodity prices have mean zero and unit variance, and then impulse response functions are appropriately re-scaled with the ratio between standardized and non-

\footnotetext{
${ }^{1}$ The policy variable such as federal fund rate is usually considered in the macroeconomic literature.
} 
standardized dataset.

The transition equation is given by

$$
Z_{t}=C_{t}+\sum_{j=1}^{P} \beta_{t j} Z_{t-j}+\sum_{j=0}^{J} \gamma_{t j} \ln \zeta_{t-j}+\Omega_{t}^{1 / 2} e_{t}, \quad \text { with } \quad e_{t} \sim \mathbb{N}(0, I)
$$

where

$$
Z_{t}=\left[\begin{array}{lll}
T_{t} & F_{t}
\end{array}\right]^{\prime}, \quad \Omega_{t}=A_{t}^{-1} H_{t} A_{t}^{-1^{\prime}} \quad \text { where } A_{t} \text { is lower triangular matrix given by }
$$

$$
A_{t}=\left(\begin{array}{cccc}
1 & 0 & 0 & 0 \\
a_{21 t} & 1 & 0 & 0 \\
\vdots & \vdots & \ddots & \vdots \\
a_{N 1 t} & a_{N 2 t} & \ldots & 1
\end{array}\right)
$$

Each non-zero element of $A_{t}$ follows the random walk process given by

$$
a_{t}=a_{t-1}+g_{t}, \quad \operatorname{var}\left(g_{t}\right)=G
$$

The time-varying parameters in the transition equation follow the random walk process given by

$$
B_{t}=B_{t-1}+\eta_{t}, \quad \operatorname{var}\left(\eta_{t}\right)=Q_{B}
$$

where $B=\operatorname{vec}[C ; \beta ; \gamma]$. Note that since the climate variable is treated as the most exogenous variable in the literature, I put the climate variable $T_{t}$ at the first order in the transition equation (22). The number of lags for endogenous variables $P$ is set to be 1 by BIC, indicating that the dependency of the endogenous variables does not exceed one month. The number of lags for stochastic volatility terms $J$ is set to be 1 , indicating that the effect of the climate uncertainty does not exceed one month. ${ }^{2}$ By

\footnotetext{
${ }^{2}$ Increasing the number of lags $P_{2}$ does not change the estimation results significantly, but deteriorates the stability of VAR estimates.
} 
letting $A_{t}$ and $B_{t}$ follow the random walk process, moreover, I allow the permanent changes or structural breaks in the relationship between macroeconomic factors and climate variables, which is reasonable given the 50-year sample period.

The volatility of the shock $e_{t}, \zeta_{t}=\left(\zeta_{1 t}, \zeta_{2 t}\right)^{\prime}$ is given by

$$
H_{t}=\left(\begin{array}{cc}
\zeta_{1 t} & 0 \\
0 & \zeta_{2 t} S
\end{array}\right)
$$

where

$$
\zeta_{2 t} S=\zeta_{2 t}\left(\begin{array}{cccc}
S_{1} & 0 & 0 & 0 \\
0 & S_{2} & 0 & 0 \\
\vdots & \vdots & \ddots & \vdots \\
0 & 0 & \ldots & S_{N-1}
\end{array}\right)
$$

which is the scaled common volatility $\zeta_{2 t}$ using the parameter matrix, $S$. The log stochastic volatility follows the $\mathrm{AR}(1)$ process, as given by

$$
\ln \zeta_{t}=\alpha+F \ln \zeta_{t-1}+\bar{\eta}_{t}, \quad \operatorname{var}\left(\bar{\eta}_{t}\right)=Q_{\zeta}
$$

Note that I follow the AR(1) log stochastic volatility scheme of Mumtaz and Theodoridis (2018) because it is implausible that a shock generated by weather prediction error can affect the economic agent's weather forecasting capacity permanently.

The model employed here is the modified version of the FAVAR with uncertainty of Mumtaz and Theodoridis (2018). Along the lines of Carriero, Clark, and Marcellino (2015), I assume that there is a common volatility of the global commodity markets. However, I separate the climate uncertainty from the common uncertainty of the global commodity markets, and then identify the effect of climate uncertainty on the global commodity markets. By reducing the number of volatilities in the mean equation of the VAR system, I not only achieve the principle of parsimony, but also 
identify the uncertainty of a variable of interested. The number of factors extracted from the global commodity markets is set to be 3 . However, the change in number of factors does not produce a significant difference in the impulse response functions.

Regarding the estimation procedure, two-step classical approach and one-step Bayesian approach can be considered. However, the two-step approach creates the problem of generated regressors, and ignoring uncertainty in factor estimates may be problematic as temporal dimension $\bar{T}$ is greater than cross-sectional dimension $N$ in my case (Bai, 2003). On the other hand, a single-step Bayesian approach can create prohibitive computational costs (Mumtaz and Surico, 2009). Since the nonlinearity and complexity in the transition equation make the classical estimation infeasible, I employ the one-step Bayesian approach with a Markov Chain Monte Carlo (MCMC) algorithm described in Mumtaz and Theodoridis (2018).

Specifically, the MCMC algorithm I employed is as follows.

- Conditional on a draw for the stochastic volatility $\zeta_{t}, Z_{t}, B_{t}$, and $G$, the timevarying elements $a_{t}$ of $A_{t}$ matrix are drawn equation by equation using the Carter and Kohn (1994) algorithm. Conditional on drawn $a_{t}$, the variance parameter is drawn from the inverse Wishart (IW) density.

- Conditional on $A_{t}$ and $\zeta_{t}$, the N-2 elements of the scale matrix $S$ is drawn from an inverse Gamma distribution.

- Conditional on $A_{t}, \zeta_{t}, S$, and $Q_{B}$, equation (22) is the time-varying parameter VAR model. Using the Carter and Kohn (1994) algorithm, $B_{t}$ is drawn. Here, the rejection sampling procedure of the time-varying parameters at each time is implemented to ensure the stationary VAR analysis. Conditional on $B_{t}$, the covariance matrix $Q_{B}$ is drawn from IW density.

- Conditional on $\zeta_{t}$, the autoregressive parameter $F$, and the variance paramter $Q_{\zeta}$ are drawn from the set of linear regressions. 
- Conditional on $F$, and $Q_{\zeta}$, the constant $\alpha$ and the unconditional mean $\frac{\alpha}{1-F}$ are drawn from the set of linear regressions.

- Conditional on $Z_{t}, B_{t}, A_{t}, S, \alpha, F$, and $Q_{\zeta}$, the stochastic volatility $\zeta_{t}$ is simulated using a date by date independence Metropolis step (Jacquier et al., 1994).

- Conditional on $Z_{t}, Q_{\Lambda}$, and $R$, the factor loading $\Lambda_{t}$ is drawn using the Carter and Kohn (1994) algorithm. Conditional on $\Lambda_{t}$, the covariance matrix $Q_{\Lambda}$ is drawn from the IW density.

- Conditional on $Z_{t}$ and $\Lambda_{t}$, the set of linear regressions is used to draw the variance parameter $R$.

- Conditional on measurement and transition equations, $F_{t}$, the subset of $Z_{t}$, is drawn using the Carter and Kohn (1994) Algorithm.

I follow the conventional normal-inverse gamma priors and starting values described in Mumtaz and Theodoridis (2018). I use 20,000 replications and base my inference on the last 5,000 replications.

To show how the climate uncertainty affects global commodity prices, I generate impulse responses to a climate uncertainty shock based on Generalized Impulse Response Analysis developed by Koop et al. (1996). Specifically, I generate a climate uncertainty increase by a positive one standard deviation shock on the equation (23). Since we can calculate the impulse responses of the common factors to an uncertainty shock, we can also calculate impulse responses of any observed variables in $X_{t}$ using estimated factor loadings. Since an uncertainty shock is identified with exogeneity from the temperature level shock, there must be no an accompanied level shock that changes the current temperature level, allowing us to identify an exogenous volatility shock. The convergence check of the MCMC algorithm is provided in Appendix. Since there are two many parameters in the model, I select only 25 parameters among 
them for space-saving. The recursive means over 5 draws of the retained draws for the parameters in the time-varying FAVAR with stochastic volatility in mean model show little fluctuation, providing support for convergence of the algorithm.

\section{The ENSO Dynamics and Its Uncertainty}

This section provides a detailed statistical analysis for the ENSO variable. Specifically, I define the short-run relationship between the ENSO variable and the global commodity markets, and address the possible statistical issues when analyzing the climate-macroeconomic relationship using the VAR model. Subsequently, I introduce the novel econometric methodology of Chang et al. (2017), and provide its statistical analysis on the ENSO variables. Lastly, I present the estimated climate uncertainty, which is free from any statistical problem addressed below.

The following data sources are employed for the analysis. To measure the ENSO fluctuation, I employ the Southern Oscillation Index (SOI) and the Sea Surface Temperature (SST) Anomaly in Niño 3.4 region, which are the commonly-employed ENSO indices in the literature. The SOI is measured to identify the large-scale patterns in air pressure occurring between the western and eastern tropical Pacific during ENSO periods. More specifically, the SOI has been calculated based on the differences in air pressure anomaly between Tahiti $\left(17.5^{\circ} \mathrm{S}, 149.6^{\circ} \mathrm{W}\right)$ and Darwin $\left(12.4^{\circ} \mathrm{S}, 130.9^{\circ} \mathrm{W}\right)$ in northern Australia. By doing so, smoothed time series of the SOI is known to approximate the changes in ocean surface temperatures across the eastern tropical Pacific.

The negative value of the SOI represents below-normal air pressure at Tahiti and above-normal air pressure at Darwin. Prolonged periods of negative SOI values coincide with El Niño episodes, which is technically defined as a positive sea surface temperature departure from normal, greater than or equal in magnitude to 0.5 degrees 
Celsius, averaged over three consecutive months in the Niño 3.4 region. On the other hand, prolonged periods of positive SOI values coincide with La Niña episodes, which is technically defined as a negative sea surface temperature departure from normal, greater than or equal in magnitude to 0.5 degrees Celsius, averaged over three consecutive months in the same area. ${ }^{3}$ The SOI and the SST anomaly data are retrieved from NOAA ESRL Physical Sciences Division. ${ }^{4}$ The time span of those data is from 1965M1 to 2017M10 (634 observations).

To investigate the climate-macroeconomic relationship, it is worth considering the frequency-wise relationship between climate events and commodity price dynamics. Roughly speaking, there are three possibilities in the temperature change. The change in temperature around the Pacific equator could be a part of a long-run movement, medium-run movement, or short-run movement in temperature dynamics. Specifically, the change in long-run movement in temperature dynamics, which is characterized by a low-frequency stochastic or deterministic trend, would not be related with global commodity markets, in the sense that the long-run trend of the global commodity markets is likely explained by technological developments or economic growth. Likewise, the change in medium-run movement in temperature dynamics, which is characterized by multidecadal cycles, would not be related with global commodity markets because multidecadal commodity price fluctuations are well-explained by the macroeconomic business cycle.

From a statistical perspective, the standard VAR modeling assumes the stationarity of the employed variables, and therefore the stability of the VAR estimation cannot be maintained from the long-run or medium-run relationship. In this light, detrending a low-frequency trend in the employed data is a critical step to conduct an appropriately designed analysis. ${ }^{5}$ More specifically, the global warming literature

\footnotetext{
${ }^{3}$ See http://www.cpc.noaa.gov/products/analysis_monitoring/ensocycle/soi.shtml for details

${ }^{4}$ https://www.esrl.noaa.gov/psd/gcos wgsp/Timeseries/SOI/

${ }^{5}$ Any meaningful multidecadal variations are not detected from the ENSO data.
} 
has addressed that there is a deterministic or stochastic long-run trend in the historical global mean temperature anomaly (Estrada et al., 2013; Kaufmann et al., 2010, 2013). Thus, the ENSO, which represents the sea surface temperature around the Pacific equator, may contain such a nonstationary trend, invalidating the stationary VAR analysis. Ignoring such nonstationary characteristics in the mean equation of the VAR system would contaminate the errors with stationary assumption. Obviously, the volatility would not be properly estimated in this case.

Note that Nam (2018) shows that the global mean temperature anomaly and total radiative forcing share a stochastic trend, suggesting the stochastic detrending method. Due to the data unavailability of the forcings at a monthly frequency, however, a deterministic trend is attempted by the linear regression of the SOI with a constant and a linear time trend, resulting that the estimate of the linear time trend is 0.0001 with t-statistics, $0.45 .^{6}$ This implies that there has been no significant warming trend around Pacific equator over the 50 years. This result is consistent with the literature that has addressed the heterogeneous local climate sensitivity, which is high at the Arctic region (polar amplification), but low at low and midlatitude regions (Boer and Yu, 2003; Miller, 2017).

All things considered, the most reasonable explanation between climate events and global commodity markets is a short-run relationship, which is the relationship between short-run components (or high-frequency components) of climate events and global commodity prices, justifying the stationary VAR analysis. Put differently, the high-frequency component in commodity price dynamics could be related to that in temperature dynamics, in the sense that the agricultural productivity this month would be affected by the weather event this month, not next month or previous month. This implies that the agricultural productivity is likely affected by a high-frequency

\footnotetext{
${ }^{6}$ The estimate of the linear time trend using sea surface temperature anomaly data is 0.0002 with t-statistics, 1.22. Note that since we use the SOI data after the year of 1965, our analysis is free from the debate of a broken slope function around the year of 1955.
} 
(e.g., month) weather event, even though the actual impact of a climate event on the commodity items could appear around a year after a shock.

It is worth pointing out two issues for the further analysis. The first issue is what frequency is appropriate to define the high-frequency component. The second issue is whether we can identify the statistical relationship between variables with different frequencies. Certainly, the 4-5 year periodic ENSO cycle is too long to be defined as the high frequency cycle. Moreover, we cannot identify the relationship if the difference of frequency between two variables is large (i.e., spurious relationship). Notice that the ENSO variable does not follow the covariance stationary process, in the sense that its mean is varying over time with a 4-5 year cycle. Nevertheless, the ENSO literature (Brunner, 2002; Ubilava, 2012, 2014, 2017; Cashin et al, 2018) has provided the effect of two cycles (4-5 year signal and high-frequency noise) of the ENSO variable on one high-frequency cycle of the global commodity markets. In such a case, it is difficult to identify the high-frequency relationship between the ENSO signal and the global commodity market because the economically meaningful relationship between a periodic ENSO signal and commodity returns becomes a difficult problem when it faces a spurious regression problem in the statistics area. More importantly, the autoregressive modeling may not be a good choice to capture such a 4-5 year ENSO signal. As a result, a simple VAR model with climate and macroeconomic variables may generate a spurious result if we ignore such a medium-run cycle only contained in the climate data.

More specifically, one of the difficulties to identify the climate-macroeconomic relationship using the VAR model is to choose the length of autoregressive lags, inherited from the relationship with different frequencies. By the construction of the VAR model, the unexplained part of the VAR model should be close to the white noise process. To do so, the climate variable requires many lags to take account of cyclicality while the macroeconomic variable typically requires a few of autoregressive 
lags. In such a case, the typical lag selection procedure, which is simply expressed as the penalty from increasing lag length minus the rewards from improved goodness of fit, suggests underestimated lags for the climate variable, and overestimated lags for the macroeconomic variable because, as the size of lag increases, the goodness of fit of the climate equation can be significantly improved over the penalty, while the goodness of fit of the macro equation barely improved, but the penalty sharply increases. This implies that the climate equation could be misspecified by the omitted variable problem, and the macro equation could be suffered from inefficiency. As a result, the persistence of the estimated log stochastic volatility of the climate equation is close to unity, implying that the log volatility process of the climate variable shows much less variation than that of the macroeconomic factors. This argument can be confirmed from the sampled variance parameter, $Q_{\zeta}$ with underestimated autoregressive lags. Furthermore, the generated stochastic volatility by the method of Jacquier, Polson, and Rossi (1994) could be unrealistically exploded and unbounded by the omitted, but persistent autoregressive variables (Kim et al., 2009). ${ }^{7}$ However, including many autoregressive lags would deteriorate the stability of the VAR system. ${ }^{8}$

Table 2 provides an interesting point on the climate-macroeconomic relationship.

Table 2: AIC and BIC values of each macroeconomic and the ENSO variables

\begin{tabular}{|c|c|c|c|c|c|c|c|c|c|c|}
\hline & \multicolumn{2}{|c|}{ SPCA1 } & \multicolumn{2}{|c|}{ SPCA2 } & \multicolumn{2}{|c|}{ SPCA3 } & \multicolumn{2}{|c|}{ Deasonalized SST } & \multicolumn{2}{|c|}{ Deasonalized SOI } \\
\hline Lag & AIC & $\mathrm{BIC}$ & AIC & BIC & AIC & $\mathrm{BIC}$ & AIC & BIC & AIC & $\mathrm{BIC}$ \\
\hline 1 & 499.9 & 504.3 & 121.1 & 125.5 & 47.5 & 51.9 & 102.5 & 106.9 & 1585.5 & 1590.0 \\
\hline 2 & 499.9 & 508.8 & 123.0 & 131.9 & 47.0 & 55.9 & -23.2 & -14.3 & 1540.2 & 1549.1 \\
\hline 3 & 501.5 & 514.8 & 122.2 & 135.6 & 48.8 & 62.1 & -35.0 & -21.7 & 1529.2 & 1542.6 \\
\hline 4 & 501.9 & 519.7 & 123.5 & 141.3 & 49.3 & 67.1 & -41.8 & -24.0 & 1528.5 & 1546.3 \\
\hline 5 & 501.5 & 523.7 & 120.6 & 142.9 & 47.5 & 69.7 & -46.9 & -24.6 & 1529.8 & 1552.0 \\
\hline 6 & 502.5 & 529.2 & 121.1 & 147.8 & 48.8 & 75.5 & -45.0 & -18.3 & 1529.8 & 1556.5 \\
\hline 7 & 503.8 & 535.0 & 119.1 & 150.3 & 49.3 & 80.5 & -47.1 & -15.9 & 1527.7 & 1558.9 \\
\hline 8 & 505.5 & 541.1 & 120.1 & 155.7 & 49.2 & 84.9 & -47.2 & -11.5 & 1528.8 & 1564.4 \\
\hline 9 & 506.5 & 546.5 & 120.0 & 160.1 & 50.2 & 90.3 & -46.0 & -5.9 & 1527.7 & 1567.8 \\
\hline 10 & 508.1 & 552.7 & 120.6 & 165.1 & 50.3 & 94.8 & -48.6 & -4.1 & 1529.6 & 1574.2 \\
\hline 11 & 509.2 & 558.1 & 122.4 & 171.4 & 48.6 & 97.5 & -47.1 & 1.8 & 1521.0 & 1569.9 \\
\hline 12 & 508.8 & 562.2 & 116.9 & 170.4 & 47.9 & 101.4 & -47.5 & 5.9 & 1519.8 & 1573.2 \\
\hline
\end{tabular}

Given that the higher values of AIC and BIC indicate a higher performance of the

\footnotetext{
${ }^{7}$ Jacquier et al. (1994) show that there are substantial biases in the parameters of the log stochastic volatility equation when the persistence $(F)$ is high and the coefficient of variation $\left(\frac{\operatorname{var}\left(\zeta_{t}\right)}{\mathbb{E}\left(\zeta_{t}\right)^{2}}\right)$ is low.

${ }^{8}$ I confirmed that the time-varying FAVAR system becomes unstable when the lag length is more than or equal to five.
} 
autoregressive lags, the performance of autoregressive modeling is quite different between first three static factors and the ENSO variables. Interestingly, two popular ENSO variables stand at both extreme, in the sense that the signal to noise ratio of the SOI data is too low, and that of the SST anomaly data is too high, compared to the static macroeconomic factors. As a result, the performance of autoregressive modeling turns out to be the best for the SOI data and then first three static factors, and the SST anomaly data, which implies that there are dominant high-frequency cycles that can be captured by a few of autoregressive lags, despite of the 4-5 year strong cycle in the SOI data.

Notably, the SST data chooses $P=10$ as an optimal lag based on AIC value, and more importantly, it shows very low performance of the autoregressive modeling, implying that there may not be much high frequency cycles in the SST anomaly data. Note that the chosen length of lag is expressed in Bold. Further note that the $P=10$ model implies that each equation of the transition equation of the timevarying FAVAR system has $43(=10 \times 4+2 \times 1+1)$ regressors, which are too many to hold the stability. The correlograms of each macro factor, extracted by the classical principal component analysis, and the ENSO variables in Figure 5 support this arguments. Note that the blue horizontal lines are the Bartlett's 95\% confidence bands under the null hypothesis of white noise.

Perhaps, there are two statistical solutions. The first option is to employ the decycled ENSO data for the high-frequency relationship, in the sense that the volatility may not be properly estimated by the uncaptured 4-5 year ENSO cycle in the data. $^{9}$ The second option is to include the cyclical terms (e.g., a fourier series) in the conditional mean and conditional variance equations of the VAR model, in order to capture the cyclicality of the climate variable (Campbell and Diebold, 2005). In this paper, however, I generate a new variable using a newly developed endogenous

\footnotetext{
${ }^{9}$ The yearly analysis can also alleviate the lag length selection problem. With such low-frequency data, however, the data span is not long enough to conduct analysis.
} 

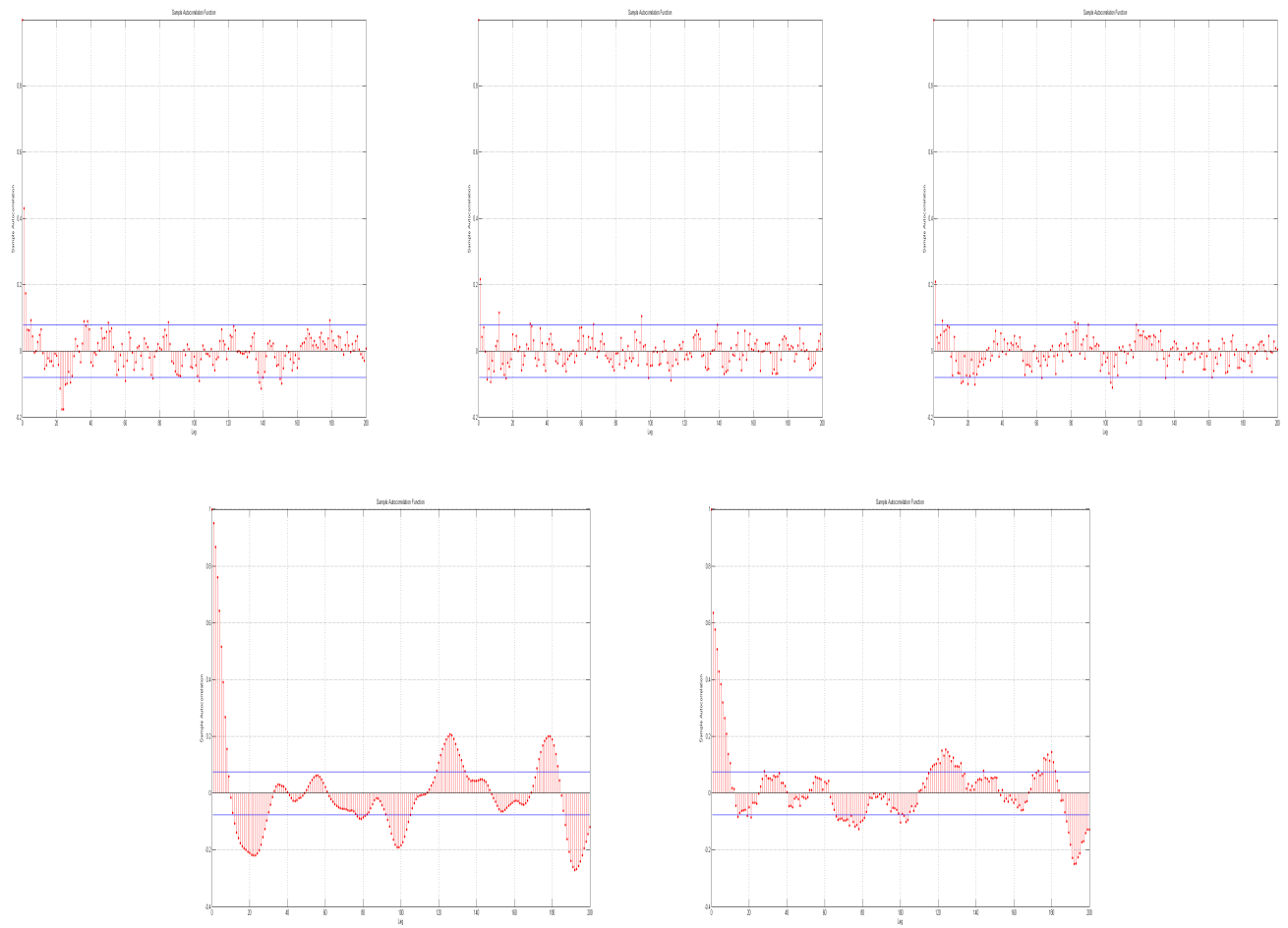

Figure 5: The Correlogram of each macroeconomic factor and SOI and SST anomaly data (clockwise) 
regime switching approach of Chang et al. (2017), because the ENSO cycle itself is the important information to generate unexpected temperature events, and including the cyclical terms could make the stationary VAR analysis too much complicated in an infeasible way.

In the way that an autoregressive latent factor determines the regimes of the climate data, the endogenous regime switching technology is exploited to transform the problematic periodicity in the climate data into the innocuous covariance-stationary high-frequency cycle. By converting the periodic ENSO data into a macro-type autoregressive process with the endogenous regime switching technology, I remove a higher order sample autocorrelation in the ENSO data. In the meanwhile, although its degree would depend on the data, I preserve information about the periodic ENSO cycle in a different way, in the sense that the extracted covariance-stationary autoregressive process only contains two-state regime information without high-frequency noises contained in the raw data. This data transformation enables us to analyze the high-frequency relationship between the 4-5 year ENSO cycle and the global commodity prices using the stationary VAR model.

The endogenous regime switching in mean model of Chang et al. (2017) is given by

$$
\gamma(L)\left(y_{t}-\mu_{t}\right)=\sigma u_{t}
$$

where $\gamma(z)=1-\gamma_{1} z-\cdots-\gamma_{k} z^{k}$ is a $k$-th order polynomial, $\mu_{t}=\mu\left(s_{t}\right), s_{t}=1\left\{T_{t} \geq \tau\right\}$, and $T_{t}=\alpha T_{t-1}+v_{t}$ with

$$
\left(\begin{array}{c}
u_{t} \\
v_{t+1}
\end{array}\right)={ }_{d} \mathbb{N}\left(\left(\begin{array}{l}
0 \\
0
\end{array}\right),\left(\begin{array}{ll}
1 & \rho \\
\rho & 1
\end{array}\right)\right)
$$

The $\mathrm{AR}(4)$ endogenous regime switching in mean model needs ten parameters to be estimated using modified Markov switching filter, requiring the computationally 
burdensome estimation procedure. To achieve a global maximizer, I utilize both profile likelihood surface algorithm and Global and Multistart search algorithms, which are explained in Appendix. Note that they call this endogenous regime switching model because a shock $\left(u_{t}\right)$ to observed time series $\left(y_{t}\right)$ at time $t$ affects the regime at time $t+1$ with an endogeneity parameter, $\rho$. It is worth noting that the extracted factor $T_{t}$ determines time periods of high-low (El Niño and La Niña) for the ENSO cycle. In this light, I call the extracted factor from the ENSO variable the ENSO factor. Obviously, we may need $N$-state regime switching framework to reflect the smoothly changing mean of the ENSO dynamics. At least, the ENSO dynamics is well-represented by three states; El Niño state, La Niña state, and neutral state. I leave it for future research.

Notice that both agricultural commodity return and sea surface temperature around Pacific equator may have a predictable seasonality, implying that their might be a spurious correlation coming from the position of the Earth from the Sun, not from a difference in ocean pressure causing the ENSO pattern. To remove such a spurious relationship, I deseasonalize the SOI and SST anomaly data by obtaining least squares residuals from the regression of each ENSO variable on twelve dummy variables for each month of the year. It is worth noting that the SOI data has some seasonality ${ }^{10}$ while the SST anomaly data has no significant seasonality. Figure 6 displays the deseasonalized SOI and SST anomaly data, and their extracted factors.

The estimates for the deseasonalized SOI and the deseasonalized SST anomaly data from the $\mathrm{AR}(4)$ endogenous regime switching in mean model are in Tables $3 .{ }^{11}$ All parameters of the employed model are generally significant. The parameters, $\underline{\mu}$ and $\bar{\mu}$ indicate mean values of low and high states of the ENSO variable, identifying that mean values of the high state of the SOI and the SST are 0.11 and 0.05 , and

\footnotetext{
${ }^{10}$ Three dummy variables out of twelve dummies are statistically significant at $5 \%$ level.

${ }^{11}$ The endogenous regime switching in mean model with higher order autoregressive lags will be attempted later.
} 

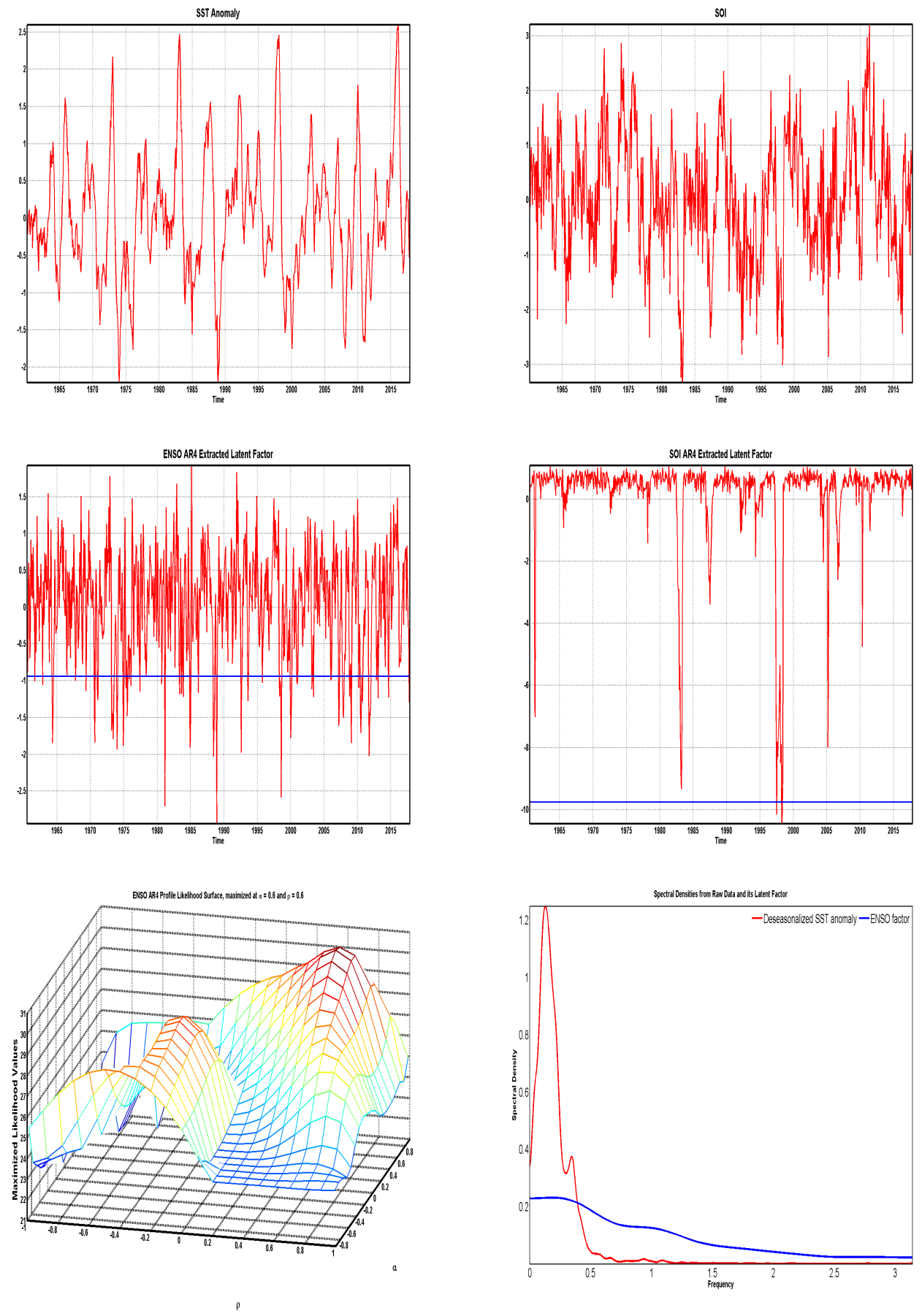

Figure 6: Deseasonalized SST anomaly and SOI data, their extracted factors, and profile likelihood surface and spectral densities for the deseasonalized SST anomaly 
those of the low state of the SOI and the SST are -2.02 and $-0.21{ }^{12}$ Note that the high state of the SOI is La Niña and the low state of the SOI is El Niño while the high state of the SST is El Niño and the low state of the SST is La Niña. The extracted factor, $T_{t}$ is interpreted as the indicator of the ENSO cycle, which follows a stationary process having zero mean and unit variance, and therefore the estimates of the parameter $\tau,-9.75$ for the SOI, and -0.94 for the SST do not represent a value of the ENSO variable, but represent a value of regime threshold of the ENSO cycle indicator. Interestingly, the estimates of the parameter $\tau$, which are marked with a blue line in Figure 6, show that the SST anomaly data has frequently changed their two regimes (El Niño and La Niña).

Specifically, identified low regime periods by the endogenous regime switching model roughly correspond to the actual La Niña periods (70-71, 73-74, 75-76, 88-89, 99-00, 07-08, 10-11, 11-12; see Figure 8 for details). On the contrary, the SOI data has only one low-regime period (Super El Niño in 1998), implying that the SOI data may be problematic under the climatic viewpoint. This may be due to the fact that the SOI has too much noises, making difficult identify two regimes. As illustrated in Figure 6, moreover, the profile likelihood surface, which shows maximized log-likelihood values across $\alpha$ and $\rho$ by means of concentrated maximum likelihood estimation, proves that the estimation of $\mathrm{AR}(4)$ endogenous regime switching in mean model for the deseasonalized SST anomaly identifies a global maximum, and clearly describes how the persistence and endogeneity are important to understand the ENSO cycle. In this light, I employ the ENSO factor extracted from the deseasonalized SST anomaly data for the subsequent analysis and the empirical results from the time-varying FAVAR with uncertainty model in Section 2 (i.e., $T_{t}$ in equations $\left.(20)-(22)\right) .{ }^{13}$

\footnotetext{
${ }^{12}$ For the expositional convenience, I simply use the terminology, the SOI and the SST by suppressing the words, deseasonalization and anomaly.

${ }^{13}$ For the stability condition of the SST anomaly, the characteristic polynomial is $(1-1.2 x-$ $\left.0.04 x^{2}+0.25 x^{3}+0.07 x^{4}\right)=0$ whose solution does not exist. However, two following necessary conditions for stationarity are satisfied; $1.2+0.04-0.25-0.07=0.92<1$ and $|-0.07|<1$.
} 
The estimates of the parameters, $\alpha$ and $\rho$, which indicate the degree of persistence of the ENSO cycle and the degree of endogeneity, reveal several interesting points for the ENSO variable. First, the degree of persistence of the ENSO cycle (0.62) is not high, implying a predictable regime switching process, and in particular, its sign is positive, meaning that a positive SST anomaly typically brings a positive SST anomaly. Secondly, the endogeneity of regime change is estimated by 0.61 , implying that only $61 \%$ of a shock at time $t$ to the SST anomaly $y_{t}$ is exploited to determine the regime switching at time $t+1$. As indicated by the estimates of the $\mathrm{AR}(4)$ parameters $\gamma(1.2,0.04,-0.25,-0.07)$, the SST anomaly follows a reverting process to both its state-dependent mean $\left(\mu_{t}\right)$ and its global mean $\mathbb{E}\left[y_{t}\right]$. As explained by Chang et al. (2017) for the case of a positive estimate $\hat{\rho}$, however, a positive shock $u_{t}$, albeit it makes $y_{t}$ revert to the state-dependent mean $\mu_{t}$, increases the probability of having high regime in the state-dependent mean $\mu_{t+1}$ of $y_{t+1}$, and therefore the state-dependent mean $\left(\mu_{t}\right)$ moves to anti-reverting direction. Further, the regime switching is more likely to occur if $y_{t}$ is between the two state-dependent means.

More importantly, the estimated spectral densities in Figure 6 illustrate the role of endogenous regime switching approach. Note that the area under the spectral curve at the domain $[0, \pi]$ represents the half of the variance of the data, and therefore there must be a scaling factor to compare two spectral densities. Specifically, the variances of the deseasonalized SST anomaly and the ENSO factor are 0.713 and 0.583 , which means that a direct comparison may exaggerate the values of the estimated spectral density of the raw data. In such a reason, I multiply a scaling value, 0.817 to the estimated spectral density of the raw data, so that the areas of two estimated spectral densities are the same. Notice that two spectral densities intersect at the frequency, 16.11 month, indicating that the endogenous regime switching technology substantially attenuates the ENSO dynamics of lower than 16.11 month frequency by about 
$66 \%\left(=100 \times \frac{0.2634-0.0890}{0.2634}\right) \cdot^{14}$

Although the estimated spectral density indicates there are little high-frequency noises in the raw data, the endogenous regime switching technology gets rid of highfrequency noises in the raw data, and transforms a 4-5 year signal of the raw data into the high-frequency cycle. In this light, the amount of attenuated low-frequency dynamics (i.e., 4-5 year ENSO cycle) could be re-expressed with the amplified highfrequency dynamics (greater than 16.11 month frequency). By doing so, the amplified high-frequency dynamics contain the low-frequency information with an estimated regime threshold value, $\hat{\tau}$, in the sense that a low value of the ENSO factor likely corresponds to the La Niña state.

Table 3: ERS AR(4) estimates for deseasonalized SOI and SST anomaly

\begin{tabular}{rrrrr}
\hline \hline & SOI Est. & S.E. & SST Est. & S.E. \\
\hline$\underline{\mu}$ & -2.0168 & 0.1000 & -0.2058 & 0.0850 \\
$\bar{\mu}$ & 0.1075 & 0.0678 & 0.0545 & 0.0834 \\
$\sigma$ & -9.7519 & 0.4245 & -0.9394 & 0.1234 \\
$\gamma_{1}$ & 0.7628 & 0.0148 & 0.1971 & 0.0084 \\
$\gamma_{2}$ & 0.4128 & 0.0548 & 1.1999 & 0.0371 \\
$\gamma_{3}$ & 0.1903 & 0.0320 & 0.0420 & 0.0534 \\
$\gamma_{4}$ & 0.0361 & 0.01961 & -0.2543 & 0.0405 \\
$\alpha$ & 0.9857 & 0.0063 & -0.0677 & 0.0293 \\
$\rho$ & -0.2573 & 0.0859 & 0.6083 & 0.1270 \\
$1 \mathrm{lk}$ & -822.7451 & & 30.7484 & \\
\hline
\end{tabular}

\footnotetext{
${ }^{14}$ Each value is calculated using the Riemann sum at the domain, $[0,0.39]$.
} 
Fully taking account of all possible statistical factors enables us to analyze the uncertainty in the climate event, in the sense that the climate uncertainty is defined as the conditional standard deviation of the one-period-ahead unforecastable component of the ENSO factor. Figure 7 shows the estimated climate uncertainty (i.e., $\hat{\zeta}_{1 t}$ of equation (23)) with $68 \%$ error bands. According to the NOAA record, ${ }^{15}$ there have been four super El Niño periods after 1968 (1972-73, 1982-83, 1997-98, 201516), which are marked in Figures 7 - 8. Roughly, the uncertainty of the ENSO factor measured by the stochastic volatility of the model prediction residual has risen during super El Niño periods.

It is important to note that Campbell and Diebold (2005) provide the statistical analysis for the climate risk (or weather risk). More specifically, Campbell and Diebold (2005) provide a standard temperature modeling, which captures a deterministic trend using time trends, periodicity using a fourier series, and high-frequency cycles using autoregressive lags. Statistically, the temperature dynamics can be written as

$$
\begin{aligned}
\text { Temp }_{t}= & \sum_{i=0}^{I} \alpha_{i} t^{i}+\sum_{j=1}^{J}\left(\beta_{1 j} \cos 2 j \pi\left(\frac{t}{T}\right)+\beta_{2 j} \sin 2 j \pi\left(\frac{t}{T}\right)\right)+\sum_{p=1}^{P} \gamma_{p} T e m p_{t-p} \\
& +\sigma_{t} \varepsilon_{t}
\end{aligned}
$$

Note that the deterministic volatility $\sigma_{t}$ captures periodic variations and high-frequency cycles of unpredictable components under the GARCH framework. Applying equation (24) to the sea surface temperature data, the designed periodicity and high-frequency cycle may correspond to 4-5 year ENSO cycle and monthly temperature variations, respectively. Given that the macroeconomic factors have a low predictability for the climate variable, it is worth comparing the estimated climate uncertainty from the ENSO factor with that of Campbell and Diebold (2005). Note that the climate un-

\footnotetext{
${ }^{15}$ http://ggweather.com/enso/oni.htm
} 
certainty from the ENSO factor is generated by the unforecastable component of the two-state regime switching process. On the contrary, the climate risk of Campbell and Diebold (2005) is generated from not only the uncertainty of the continuouslychanging regime switching process, but also the uncertainty of the high-frequency variations. In this light, we may conjecture that the uncertainty generated by twostate regime-switching process in this paper is greater than that by the continuouslychanging regime switching process. However, the magnitude comparison between two different approaches is difficult if we assume that the uncertainty also comes from the high-frequency variations. The detailed analysis for the comparison is loaded in the appendix.

Notice that it may be questionable that the climate uncertainty is generated

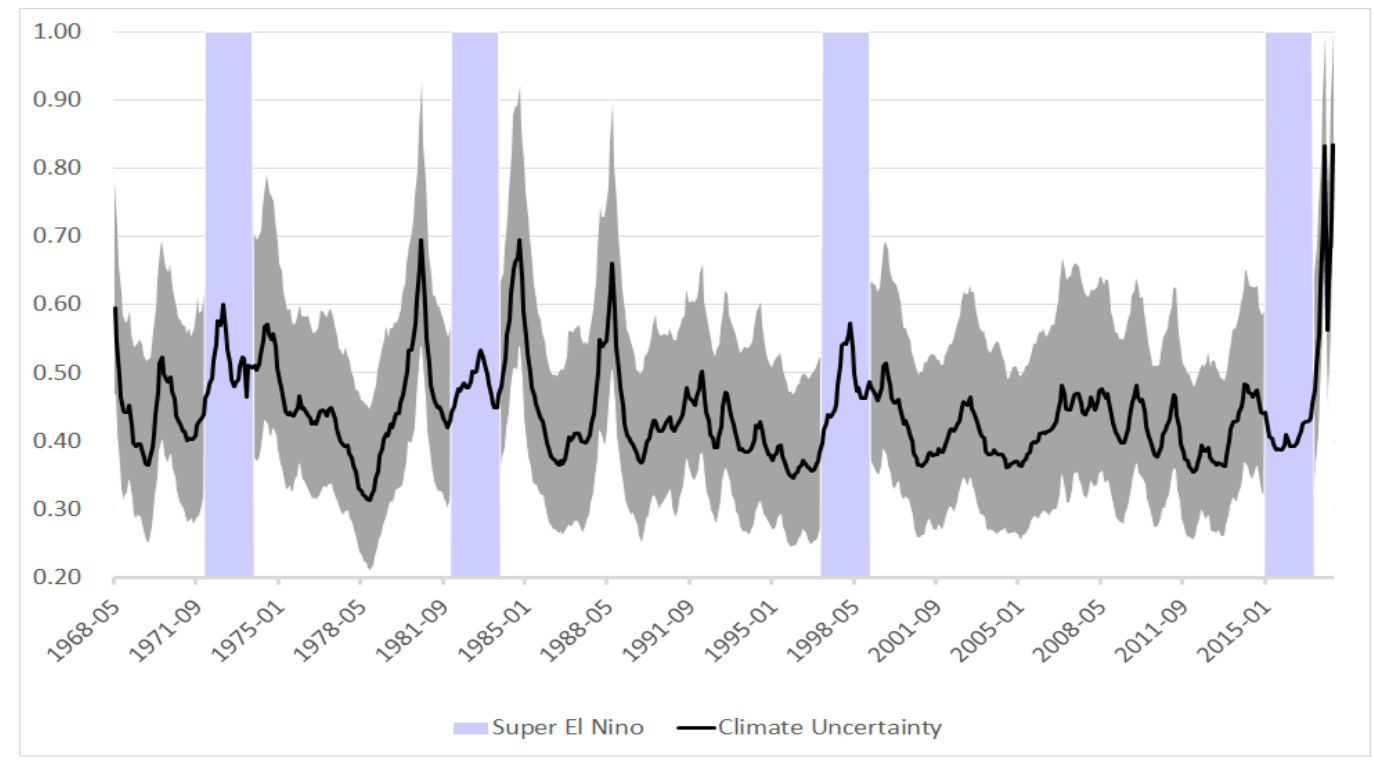

Figure 7: Climate Uncertainty with 68\% Error Bands

from one-month-ahead forecasting procedure. Since the ENSO affects the global climate system, the climate scientist has been interested in one-year-ahead future ENSO events. However, since the signal to noise ratio of the equatorial Pacific temperature between March and May has been very low (Goddard et al., 2001), this "springtime barrier" has shortened the ENSO forecasting horizon, acknowledging that the fore- 


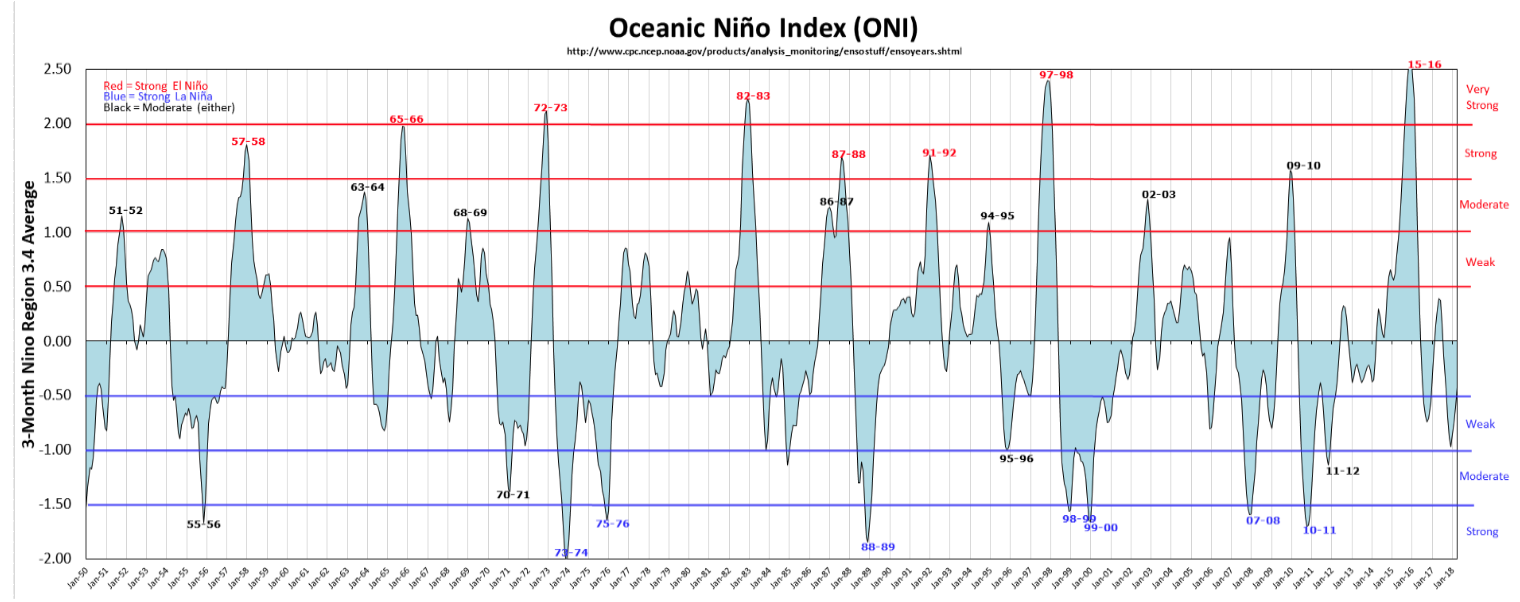

Figure 8: ENSO Index

casting horizon of both statistical and climate models has been limited to 6 month ahead. In the meanwhile, this implies that the climate uncertainty can be more realistically generated from 6 month ahead forecasting errors, which is a longer horizon than I defined. Given that the forecasting errors typically increase as the forecasting horizon increases, the one-month-ahead climate uncertainty may underestimate the true uncertainty effect on the global commodity markets. To address such a practical issue, this paper allows the one-month-ahead climate uncertainties generated for the past few months to affect the current global commodity markets. These onemonth-ahead forecasting errors for a few month could reduce the discrepancy between forecasting errors from a longer horizon and those from one month horizon.

Specifically, 2-month-ahead forecasting error can be decomposed as

$$
\mathbb{E}\left[T_{t+2}-T_{t} \mid \mathcal{F}_{t}\right]=\mathbb{E}\left[T_{t+2}-T_{t+1} \mid \mathcal{F}_{t}\right]+\mathbb{E}\left[T_{t+1}-T_{t} \mid \mathcal{F}_{t}\right]
$$

and one-month-ahead recursive forecasting error is

$$
\mathbb{E}\left[T_{t+2}-T_{t+1} \mid \mathcal{F}_{t+1}\right]+\mathbb{E}\left[T_{t+1}-T_{t} \mid \mathcal{F}_{t}\right] .
$$


Therefore, the gap between 2-month-ahead forecasting error and one-month-ahead recursive forecasting error is

$$
\mathbb{E}\left[T_{t+2} \mid \mathcal{F}_{t+1}\right]-\mathbb{E}\left[T_{t+2} \mid \mathcal{F}_{t}\right]+\mathbb{E}\left[T_{t+1} \mid \mathcal{F}_{t}\right]-T_{t+1}
$$

The first term is generated by the information gap between time $t+1$ and time $t$ when forecasting $T_{t+2}$, and the second term is generated by the information gap between time $t+1$ and time $t$ when forecasting $T_{t+1}$. Acknowledging the fact that the new information for one month would be too small to improve the forecasting accuracy, this paper applies the one-month-ahead recursive forecasting error scheme. Since I employ the large-scale FAVAR system, however, including too many one-month-ahead uncertainties in the FAVAR transition equation may deteriorate the stability of the whole FAVAR system (i.e., the lag length $J$ of equation (22)). Possibly, plugging the accumulated one-month-ahead uncertainties into the FAVAR transition equation (i.e., $\gamma_{t} \ln \left(\sum_{j=0}^{J} \zeta_{t-j}\right)$ in equation $\left.(22)\right)$ could be considered. However, it is implausible to impose a same coefficient for the uncertainty of this month and that of six month before. I leave the detailed examination for future research.

One may think that the unobserved component model can achieve the same goal, in the sense that the unobserved factor is assumed to follow the $\mathrm{AR}(1)$ process with zero mean and constant variance. However, the conventional unobserved component model assumes that there is only one regime in the observed data, and ignoring multi-state regime switching framework in the observed data would fail to remove the higher-order sample autocorrelation. In such a case, the statistical problem mentioned above still remained. The detailed analysis is loaded in the appendix. 


\section{The Global Commodity Markets and Identifying Their Commonality}

This section provides the dynamic factor analyses of the global commodity markets using two different classical component analyses. By doing so, I identify the commonalities of the global commodity markets. In addition, I present the estimated market uncertainty, which is a common stochastic volatility of the macroeconomic equations. The 63 global commodity items are collected from World Bank Global Economic Monitor (GEM) Commodities website, which are listed in appendix (Table 6). ${ }^{16}$ The time span of commodity prices is from 1965M1 to 2017M10 (634 observations). ${ }^{17}$ The downloaded nominal prices are deflated using the U.S. producer price index (PPI), collected from the St. Louis Fed, and then I take the natural logarithms to obtain the log real price. For the stationary transformation of log real commodity prices, I employ the first difference (FD) filter, providing the interpretation of the commodity returns or percentage difference from its lagged variable.

One may consider the Hodrick-Prescott (HP) filter with a $\lambda$ value, 129,600, which extracts the business cycle frequency (6-32 quarters) of the data, in the sense that the business cycle frequency is similar with the ENSO cycle. However, I employ the FD filter because the HP filter smooths out the high-frequency cycles in the commodity data, which are essential information to identify the high-frequency relationship. It is worth noting that the ENSO literature has provided the empirical findings that the climate events would affect the high-frequency commodity price dynamics (i.e., the change in price from last month). Furthermore, the impulse responses significantly generated from the HP filtered data typically last longer than two years, which are not sensible under agricultural viewpoint. In addition, there have been some concerns

\footnotetext{
${ }^{16} \mathrm{http}$ // databank.worldbank.org/data/reports.aspx?source=global-economic-monitorcommodities

${ }^{17}$ The data is available from 1960M1. However, I discard first 5-year observations due to data credibility.
} 
about the spurious dynamics raised from the HP-filter in the literature (Hamilton, 2018). Moreover, it is difficult to maintain a stability of the time-varying FAVAR system with the HP-filtered data.

The factor analysis assumes that there are a small number of factors that represent the commonality in the global commodity markets. The idiosyncratic component is the orthogonal component to the common component, and designed to explain the variable-specific movement. As the extracted commonalities from a large panel of commodity markets are exploited to overcome omitted variable problem, the model employed should be correctly specified. To derive unobservable factors or common components, I employ the Bayesian dynamic factor approach with time-varying factor loadings, in order to take account of uncertainty in factors and factor loadings (Delnegro and Otrok, 2005). However, since the extracted common factors are known to show quite similar movements with the factors extracted from principal component analyses, I exploit the classical principal component analyses to identify the commonality in the global commodity markets through this Section (See Figure 10).

Stock and Watson (2002) employ the static principal component analysis (or static factor), which finds the linear combinations of the large dataset $X_{t}=\left\{x_{1 t}, x_{2 t}, \ldots, x_{N t}\right\}$ that provides a maximized variance of factors. It turns out that this problem can be reformulated as a standard eigen-decomposition problem. An alternative way to extract common components is the dynamic principal component analysis (Forni et al., 2000, 2005, inter alia). The dynamic factor model differs from the static factor model in the sense that current, past, and future dynamic principal components are allowed to affect the contemporaneous variables. A drawback of the dynamic principal component analysis is that it accompanies with the estimation of multivariate spectral density, which generates another source of error. On the other hand, the static principal component analysis only considers the linear combination of contemporaneous variables $X_{t}$, which fails to consider the lead-lag relationship between the 
commodity prices.

To illustrate the dynamic factor model, I assume that we know the number of factors $q$. The dynamic factor model, developed by Brillinger (1981) and extensively studied by Forni et al. $(2000,2005)$ and Favero et al. (2005), can be represented by

$$
X_{t}=B(L) F_{t}+\xi_{t}
$$

The common component is composed of $q$-dimensional factors $F_{t}=\left(F_{1 t}, F_{2 t}, \ldots, F_{q t}\right)^{\prime}$ and their lags with their factor loadings, and $\xi_{t}$ is an idiosyncratic component, which is allowed to be cross-sectionally and weakly correlated. Note that the common component in equation (25) corresponds to $\Lambda^{F} F_{t}$ in equation (20).

To deal with the omitted variable bias in the dynamic factor model, Forni et al. (2000) provide a heuristic way to choose optimal number of factors $q^{*}$ without a formal testing procedure, which is (i) as the number of variables increases, the fist $q$ dynamic eigenvalues averaged over frequencies diverge while the $q+1$ dynamic eigenvalue averaged over frequencies is relatively stable or (ii) there is a big gap between the variance explained by the $q$ dynamic principal component and that by the $q+1$ dynamic principal component. For the comparison with static principal components, however, I extract the three dynamic principal components.

The first step is to estimate the sample multivariate periodogram of $N$ detrended log real global commodity prices, $X_{t}$, which is given by

$$
\begin{aligned}
\Sigma^{T}\left(\theta_{h}\right) & =\sum_{k=-M}^{M} \Gamma_{k}^{T} \omega_{k} e^{-i k \theta_{h}}, \quad \theta_{h}=2 \pi h /(2 M+1), h=0,1, \ldots, 2 M \\
& =\left\{\Gamma_{0}+\sum_{k=1}^{M} \omega_{k}\left(\Gamma_{k}^{T} e^{-i k \theta_{h}}+\Gamma_{k}^{T^{\prime}} e^{i k \theta_{h}}\right)\right\}
\end{aligned}
$$

where the sample covariance matrix of $X_{t}, \Gamma_{k}^{T}=T^{-1} \sum_{t=k+1}^{N}\left(X_{t}-\bar{X}\right)\left(X_{t-k}-\bar{X}\right)$ and $\omega_{k}$ is weighting coefficient from Bartlett window of size $M$. 
At each frequency $\theta_{h}$, the eigenvalues and their corresponding eigenvectors of $\Sigma^{T}\left(\theta_{h}\right)$ are obtained. Let the first $q$ eigenvalues and associated eigenvectors of $\Sigma^{T}\left(\theta_{h}\right)$ be $\lambda_{j}^{T}\left(\theta_{h}\right)$ and $p_{j}^{T}\left(\theta_{h}\right), j=1,2, \ldots, q$ for $h=0,1, \ldots, 2 M$. For inverse discrete Fourier transform, define $p_{j}^{T}(L)$ as

$$
p_{j}^{T}(L)=\sum_{k=-M}^{M} p_{j, k}^{T} L^{k}, \quad p_{j, k}^{T}=\frac{1}{2 M+1} \sum_{h=0}^{2 M} p_{j}^{T}\left(\theta_{h}\right) e^{i k \theta_{h}}, \quad k=-M, \ldots, M .
$$

Then, $p_{j}^{T}(L) X_{t}, j=1, \ldots, q$, are the first $q$ dynamic principal components of $X_{t}$. Then, the fitted value by running least squares regression of $X_{t}$ on past (lags), present, and future (leads) of each of the first $q$ dynamic principal components is the estimated common component (Forni et al., 2000). It is worth noting that the dynamic principal component analysis reduces to the static principal component analysis in case of $M=0$. In the meanwhile, the two-sided factor loading with lead and lag order $M$ creates a practical problem in the sense that both beginning and end of factors are not available. As recommended by simulation study in Forni et al. (2000), I set the length of lead-lag $M$ to be $6=\operatorname{round}\left(\frac{2}{3} T^{1 / 3}\right)$, implying that the information up to six months ahead is exploited to estimate the dynamic principal components.

To compare the performance of the dynamic factor model with that of the static factor model, the analogy of the $R^{2}$ statistic (Stock and Watson, 2002; Andrle et al., 2016) can be utilized, as given by

$$
R_{i}^{2}(q)=1-\frac{\sum_{t=1}^{T}\left(x_{i t}-\chi_{i t}^{q}\right)^{2}}{\sum_{t=1}^{T}\left(x_{i t}-\bar{x}_{i}\right)^{2}}
$$

where $\chi_{i t}^{q}$ is the estimated common component using first $q$ dynamic principal components, and $\bar{x}_{i}$ is temporal sample mean of $x_{i t}$. Figure 9 illustrates the performance using dynamic PCA and Static PCA, indicating that the degree of the commonality is quite heterogenous across the commodities. On average, first three static principal components explain about $30 \%$ of the total variation, while first three dynamic princi- 
pal components explain about $4 \%$ of the total variation. Given that the performances of two competing methods are similar with the HP filter on the log real commodity prices ${ }^{18}$ reduced persistency (or decreased signal to noise ratio) by taking FD filter substantially attenuates the lead-lag relationship of each variable and therefore that between commodity items. In this light, I employ the static principal component analysis in the sense that the lead-lag relationship between commodity returns is not strong enough to use dynamic principal components.

Figure 10 compares the first three static principal components with the median

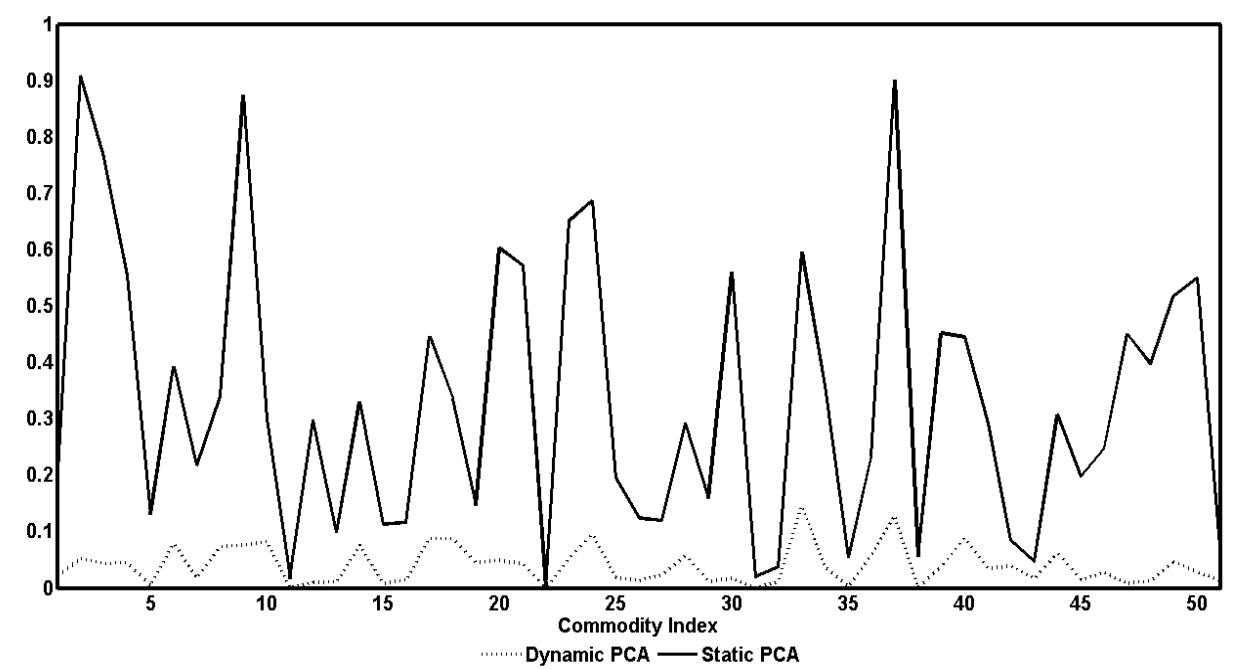

Figure 9: $R^{2}$ Comparison calculated from Dynamic PCA and Static PCA

of the posterior distribution of common factors sampled from the MCMC algorithm. As expected, the common factors extracted from the Bayesian sampling show quite similar movements with the static principal components. In particular, the simple correlation coefficients between the Bayesian factors and static factors are 0.96, 0.72, and 0.73. As the extracted common factors play an important role in understanding the FAVAR type approach, it would be an ideal step to identify the extracted static

\footnotetext{
${ }^{18}$ The first three static principal components and dynamic principal components explain $45 \%$ and $44 \%$ of the total variation, respectively.
} 


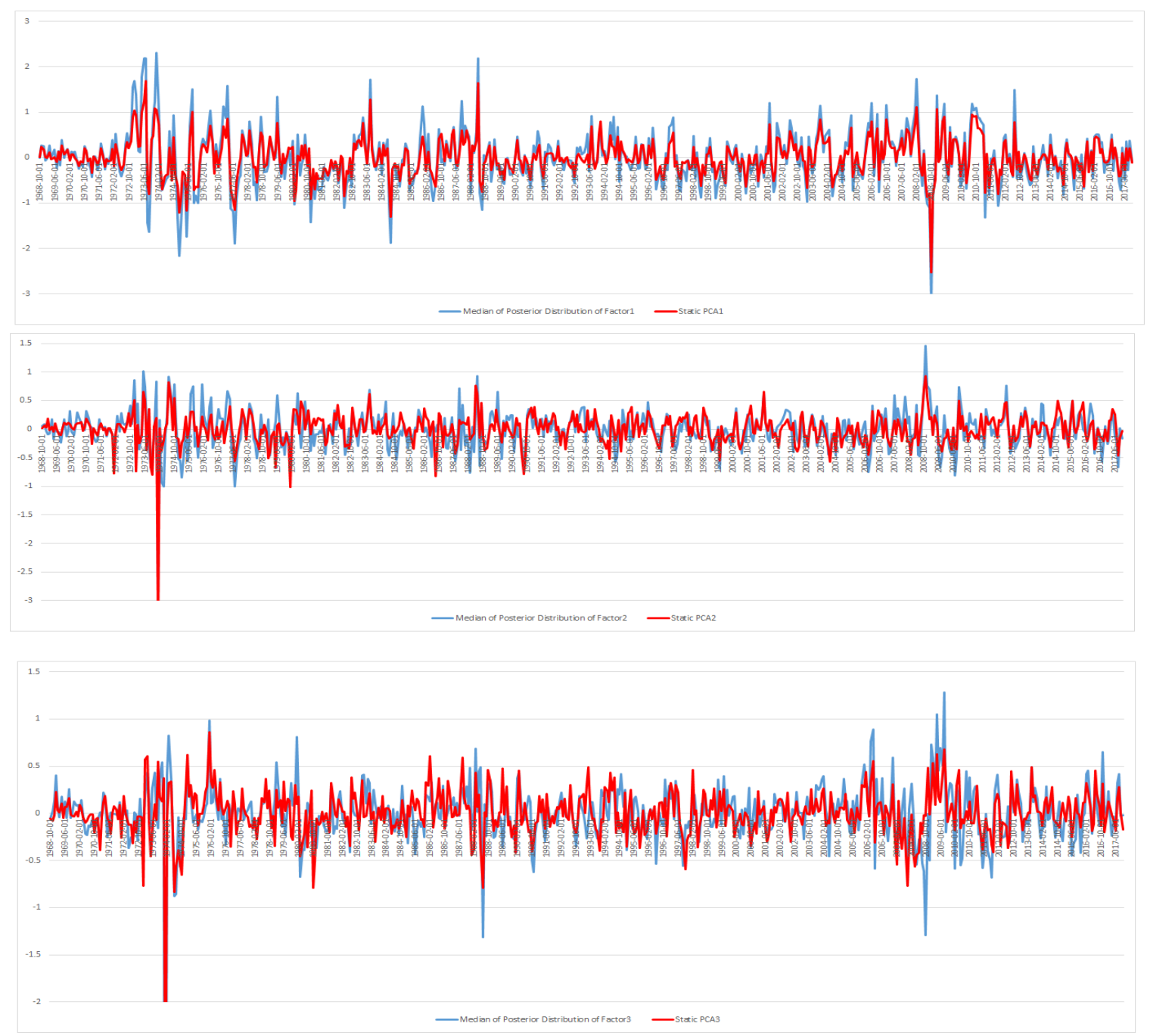

Figure 10: The Comparison between the first three static factors and the median of the posterior distribution of the common factors 
factors with the macroeconomic determinants, which is known to be a difficult task. There have been many attempts to identify the commonality in the global commodity markets by means of correlation coefficient. The commonly employed variables are the U.S. exchange rate, industrial production, real interest rate, and crude oil price (Byrne et al., 2013; Chen et al., 2014; West and Wong, 2014; Alquist and Coibion, 2014). However, there has been no clear consensus on this argument. Recently, Chiaie et al. (2017) call the extracted dynamic factor from commodity returns the global factor, in the sense that the estimated commonality is highly correlated with global economic activity (the IMF global index of commodity prices).

Following the literature, I exploit the U.S. industrial production index, crude oil price, trade weighted U.S. dollar index, global price index of all commodities, and 3 month treasury bill rate to help identify the common factors. All data are downloaded from Federal Reserve Bank of St. Louis (FRED), ${ }^{19}$ and then I take log difference on first four variables, and take first difference on 3 month treasury bill rate, which are illustrated in Figure 11. Further, I consider the deseasonalized SOI, in addition to the macroeconomic determinants. In the literature, the role of China market's economic expansion has been emphasized to explain the expanding global demand for the commodities since 2000. Due to data credibility, however, I exclude China's macroeconomic variables such as industrial production and electricity production indices in my analysis. The economic reasoning on the choice of some determinants is as follows. The lower bond return can increase the prices of commodities as an incentive for speculative or inventory demand at short-run. Further, the increase in oil price can increase the cost of commodity of energy intensive industry and decrease the relative price of biofuel production, which increase the commodity price (Byrne et al., 2013). Lastly, since most of commodities are traded in U.S. dollars, the U.S.

\footnotetext{
${ }^{19}$ The FRED codes for the U.S. industrial production index, crude oil price (WTI), trade weighted U.S. dollar index, global price index of all commodities, and 3 month T-bill are INDPRO, WTISPLC, TWEXMMTH, PALLFNFINDEXM, and TB3MS, respectively.
} 
dollar depreciation increases the commodity price to maintain the same world price (Chen et al., 2014).

Table 4 is the correlation matrix between the static factors and the selected
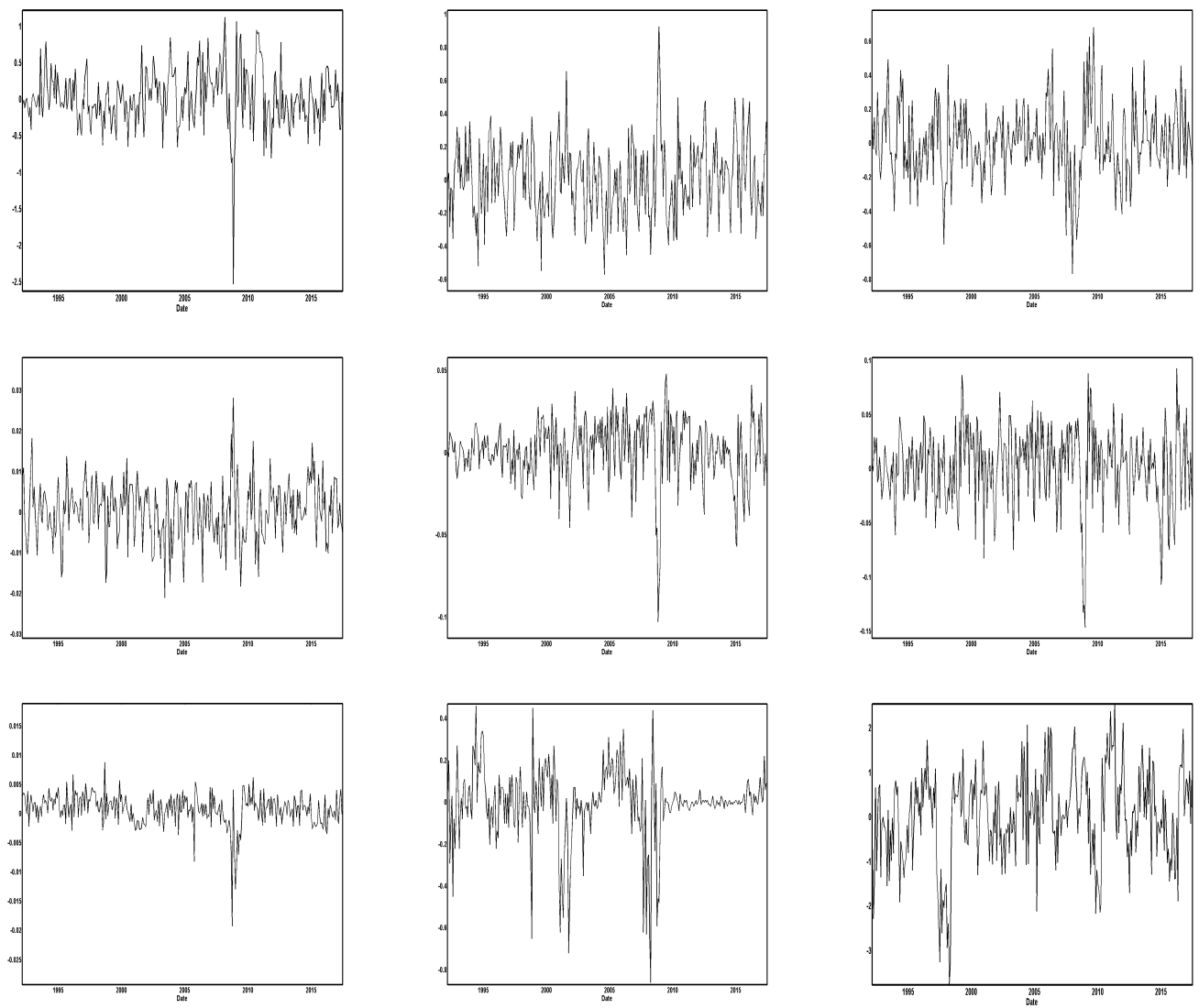

Figure 11: Top panel: the First Three Static Factors; Middle panel: the U.S. Exchange Rate Return, Global Commodity Inflation rate, Crude Oil Return; Bottom panel: the U.S. Industrial Production Growth Rate, the Change in 3 Month T-bill Rate, and deseasonalized SOI from 1992M1 to 2017M6

determinants for the period 1992M1 - 2017M6. ${ }^{20}$ At a glance, the global commodity inflation rate and crude oil return are correlated with the second factor. However, those correlation coefficients are not high enough, making difficult to correspond each determinant to each static factor. To have a better understanding, I calculate the rolling correlation coefficients between two series, and then square them (i.e., the

\footnotetext{
${ }^{20}$ The available time span across the determinants is different. Thus, I restrict the statistical analysis of this section to the time span from 1992M1 to 2017M6.
} 
Table 4: Correlation Matrix

\begin{tabular}{lrrrrrr}
\hline & US ER & \multicolumn{1}{c}{ GPI } & \multicolumn{1}{c}{ Oil } & U.S. IPI & \multicolumn{1}{c}{ Tbill } & \multicolumn{1}{c}{ SOI } \\
\hline \hline First Factor & -0.358 & 0.446 & 0.222 & -0.001 & 0.106 & 0.054 \\
Second Factor & 0.346 & -0.634 & -0.607 & -0.146 & -0.129 & 0.018 \\
Third Factor & -0.223 & 0.024 & -0.039 & 0.021 & 0.126 & -0.036 \\
\hline
\end{tabular}

rolling $R^{2}$ statistics), in the sense that each macroeconomic determinant can explain the static factors for different periods. The size of the rolling window is set to be 60 months, in the sense that the ENSO cycle is roughly about 5 years. This enables us to identify the relationship at each ENSO event. Since the rolling $R^{2}$ statistics provide how much the variability of one variable can be explained by another variable at each window, and the rolling correlation coefficients cannot provide the magnitude measure across time, the rolling $R^{2}$ coefficients are employed in my analysis.

Figure 12 shows that the rolling $R^{2}$ statistics from the regressions of each factor on the selected six determinants at each window. The dotted lines are 95\% confidence bands from 1,000 bootstrapping samples. Note that low $R^{2}$ value implies that there might be omitted variables for a target static factor, which widen the bootstrapping confidence bands. Notably, Figure 12 reveals that the selected six determinants explain each static factor in a time-heterogenous fashion. For some periods, the selected determinants fail to show a good predictability. Specifically, the third factor equation may not be significant during around year of 1998 and year of 2010. To measure the contribution of each determinant on the extracted commonalities, I estimate the marginal contribution of each determinant from total explained variation of each static factor, which I call the MC statistics. To calculate this F-type estimator, I calculate the $R^{2}$ value from all determinants and that from five determinants without the determinant of interest, and then calculate their difference relative to the $R^{2}$ value of the former. Formally, the MC statistics of the variable $x$ can be written as

$$
M C(x)=\frac{R_{\mathrm{unrest}}^{2}-R_{\mathrm{rest}}^{2}}{R_{\mathrm{unrest}}^{2}}
$$


where $R_{\text {rest }}^{2}$ is the $R^{2}$ value from the regression of a target static factor on the determinants without $x$ variable, and $R_{\text {unrest }}^{2}$ is the $R^{2}$ value from the regression of a target static factor on all determinants.

Although the MC statistics look similar with the $F$ statistics, they are different in the sense that the $F$ statistics is used to test the significance of the variable of interest, but the MC statistics is designed to measure the contribution of the variable of interest. In this light, having around zero value of the MC statistics does not mean that the determinant has no effect on the target factor. The MC statistics provide an interpretation of marginal contribution of the determinant, which other determinants cannot provide, providing clear economic interpretations. Since the selected determinants are highly correlated, the variance decomposition analysis may be invalid, in the sense that the coefficient estimates are affected by correlated determinants. Note that the correlation coefficient between global commodity inflation rate and crude oil return is 0.91 , and that between the U.S. exchange return and global price return is -0.41 . The orthogonalizing scheme may be considered. However, the order of orthogonalization scheme and its interpretation are another issues need to be solved. Moreover, repeating orthogonalizations each other could generate the unexpected estimation errors. In this light, I identify the marginal effect of each determinant.

Figure 12 illustrates that the effects of selected determinants on the first three
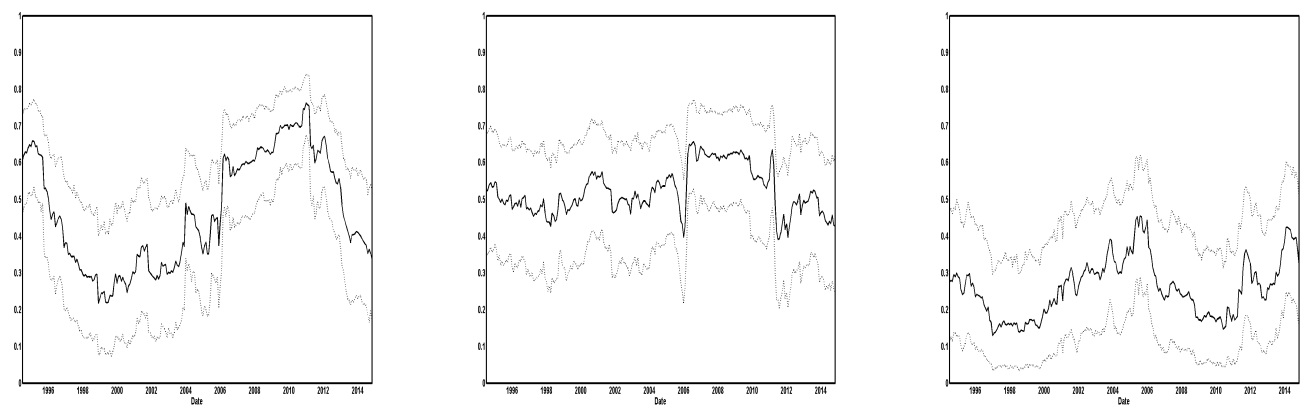

Figure 12: Rolling $R^{2}$ Statistics for the First Three Static Factors from All Determinants 
static factors are highly significant. Specifically, those determinants explain roughly about $60 \%, 50 \%$, and $30 \%$ of the total variations of the first three static factors, respectively. More specifically, Figures 13 - 15 display the MC statistics of the first three factors from the U.S. exchange rate return (left top), global commodity inflation rate (middle top), crude oil return (right top), the U.S. industrial production growth rate (left bottom), the change in 3 month T-bill (middle bottom), and the deseasonalized SOI (right bottom).

To clearly describe the contribution of each determinant on the global commod-
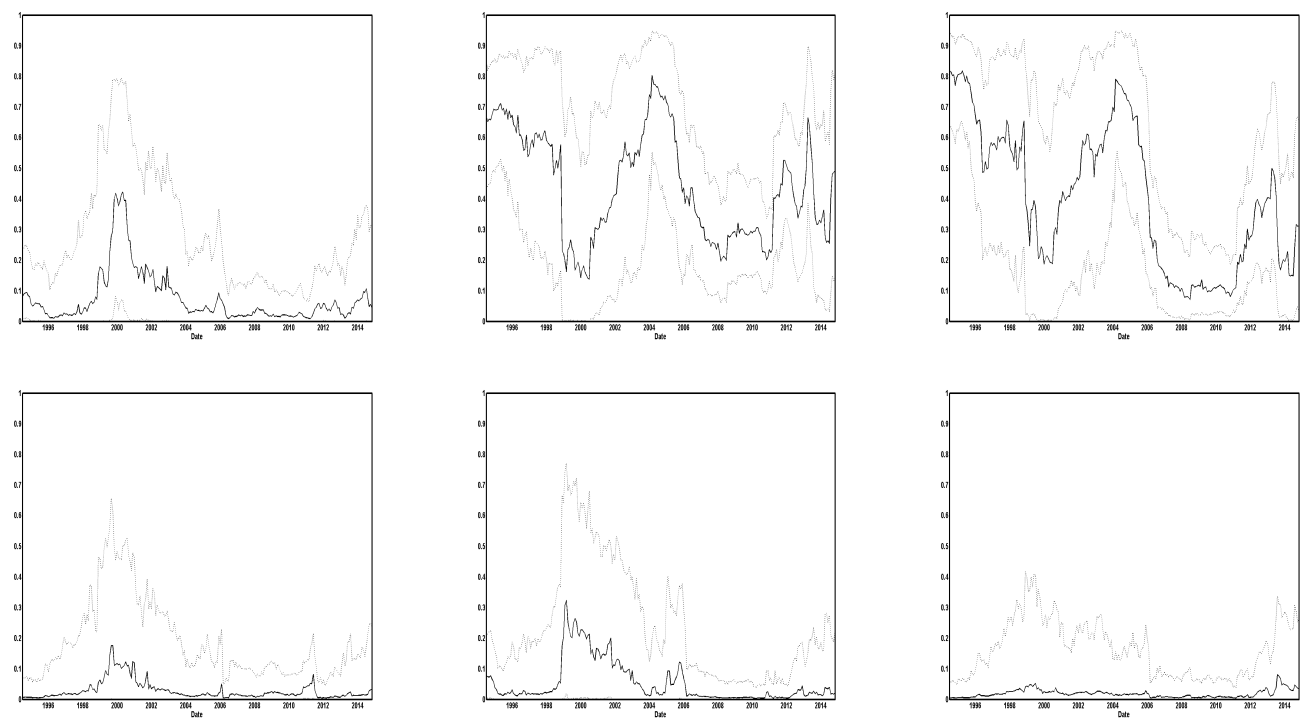

Figure 13: MC Statistics of the First Factor from Each Determinant

ity markets, I propose the following statistics. Given the average contributions of the first, second and third static factors on the total variation of the global commodity markets are $15.8 \%, 7.4 \%$, and $6.6 \%$, the contribution of each determinant, $x$ on the global commodity markets can be evaluated as follows.

$$
0.158 \times R_{1 t}^{2} \times M C_{1 t}(x)+0.074 \times R_{2 t}^{2} \times M C_{2 t}(x)+0.066 \times R_{3 t}^{2} \times M C_{3 t}(x)
$$



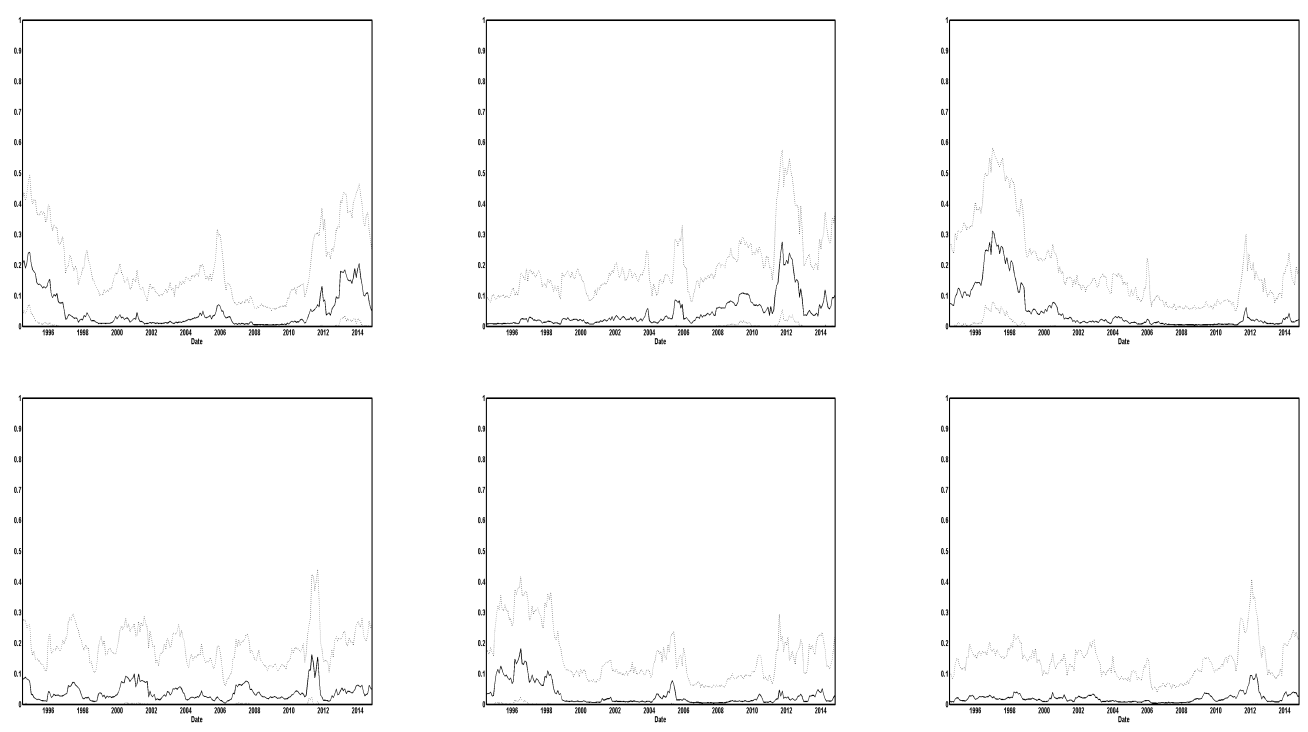

Figure 14: MC Statistics of the Second Factor from Each Determinant
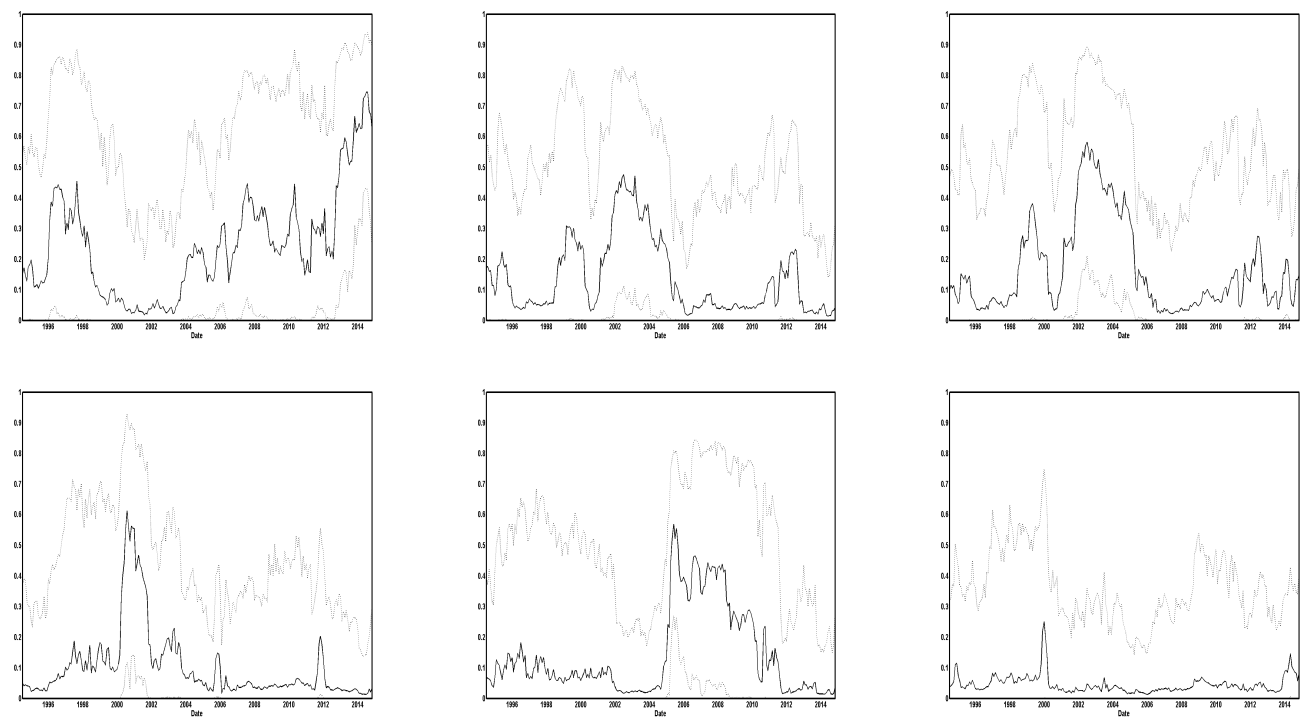

Figure 15: MC Statistics of the Third Factor from Each Determinant 
where $R_{i t}^{2}$ is the $R^{2}$ value of the $i$-th static factor using all determinants, presented in Figure 12 , and $M C_{i t}(x)$ is the MC statistics of the $i$-th static factor using a determinant $x$, presented in Figures 13 - 15 .

Figure 16 illustrates the time-varying contribution of each determinant on the global commodity markets proposed above. In overall, the U.S. exchange rate return, global commodity inflation rate, and crude oil return have bigger effects than IPI growth rate, the change in T-bill rate, and the deseasonalized SOI. In particular, the effects of the global commodity inflation rate and crude oil return become higher during the non-recession periods, implying that the changes in global fuel and non-fuel commodity prices are the main driver to move the entire global commodity markets during the non-recession periods. In other words, the influences of typical determinants addressed in the literature become weaker during the recession periods, implying that the omitted variable bias may not cause the serious problem on the estimated time-varying impulse responses during the recession periods. The effect of the U.S. exchange return has been stable over the sample period, but slightly increased for the last two years. Interestingly, the change in 3 month T-bill rate shows a relatively higher influence on the global commodity markets for the periods of 1999-2002 and 2005-2008. These two periods are known when the interest rate was relatively high, which means the higher bond return. Therefore, it decreased the commodity prices. However, when the 3 month T-bill rate was around zero rate after financial crisis in 2008, the substitutability between bond and commodity demands was disappeared. Lastly, the deseasonalized SOI level has not been effective on the global commodity markets, which is inconsistent with the ENSO literature.

As indicated by the low average explainability (30\%), the factor model does not perform well for every commodity, and therefore it is necessary to identify the commodities that work well with the factor model, in the sense that some commodities may get little benefit by incorporating complexity in the model. The black line of Fig- 

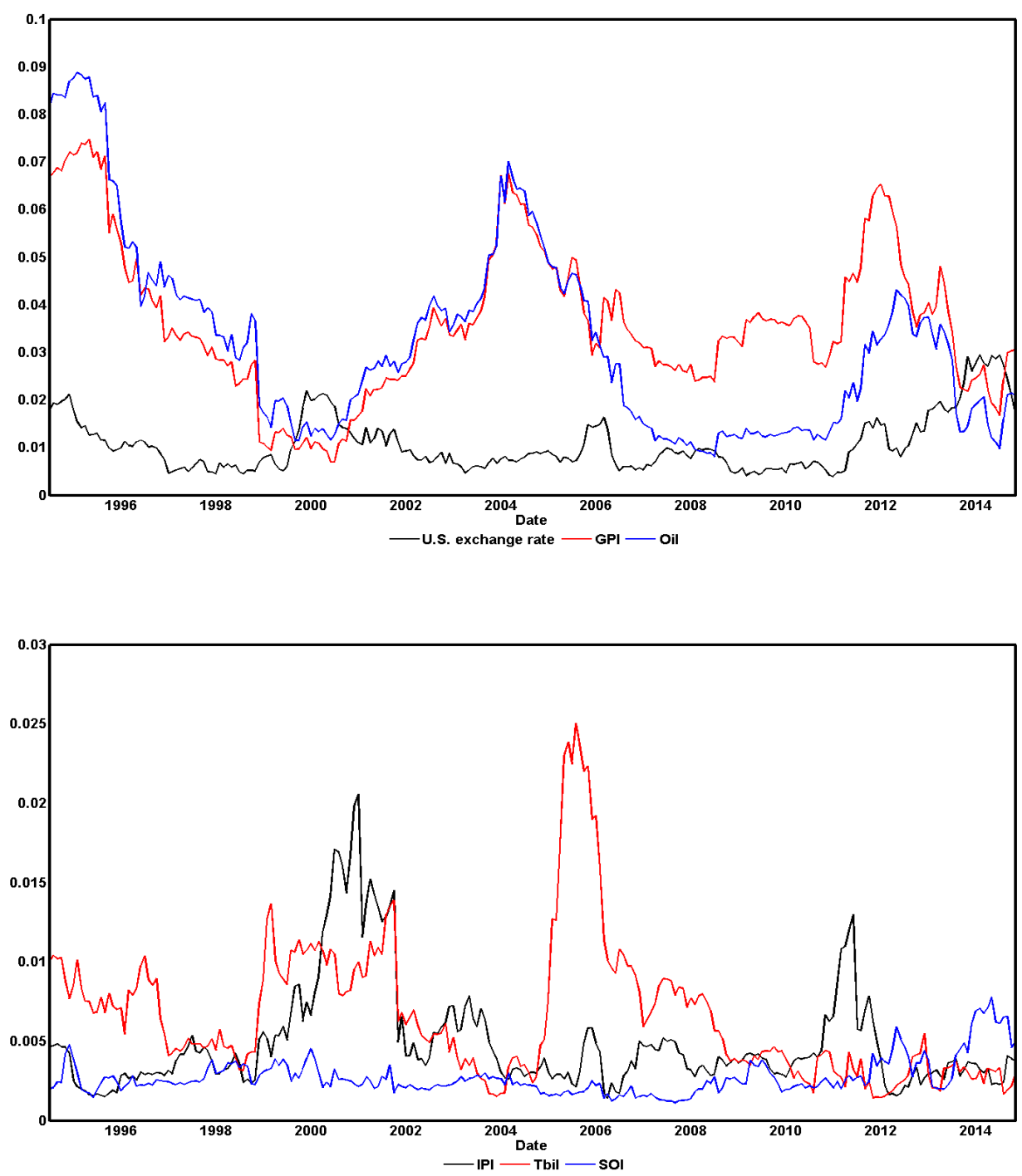

Figure 16: Time-varying Contribution of Each Determinant on the Global Commodity Markets 


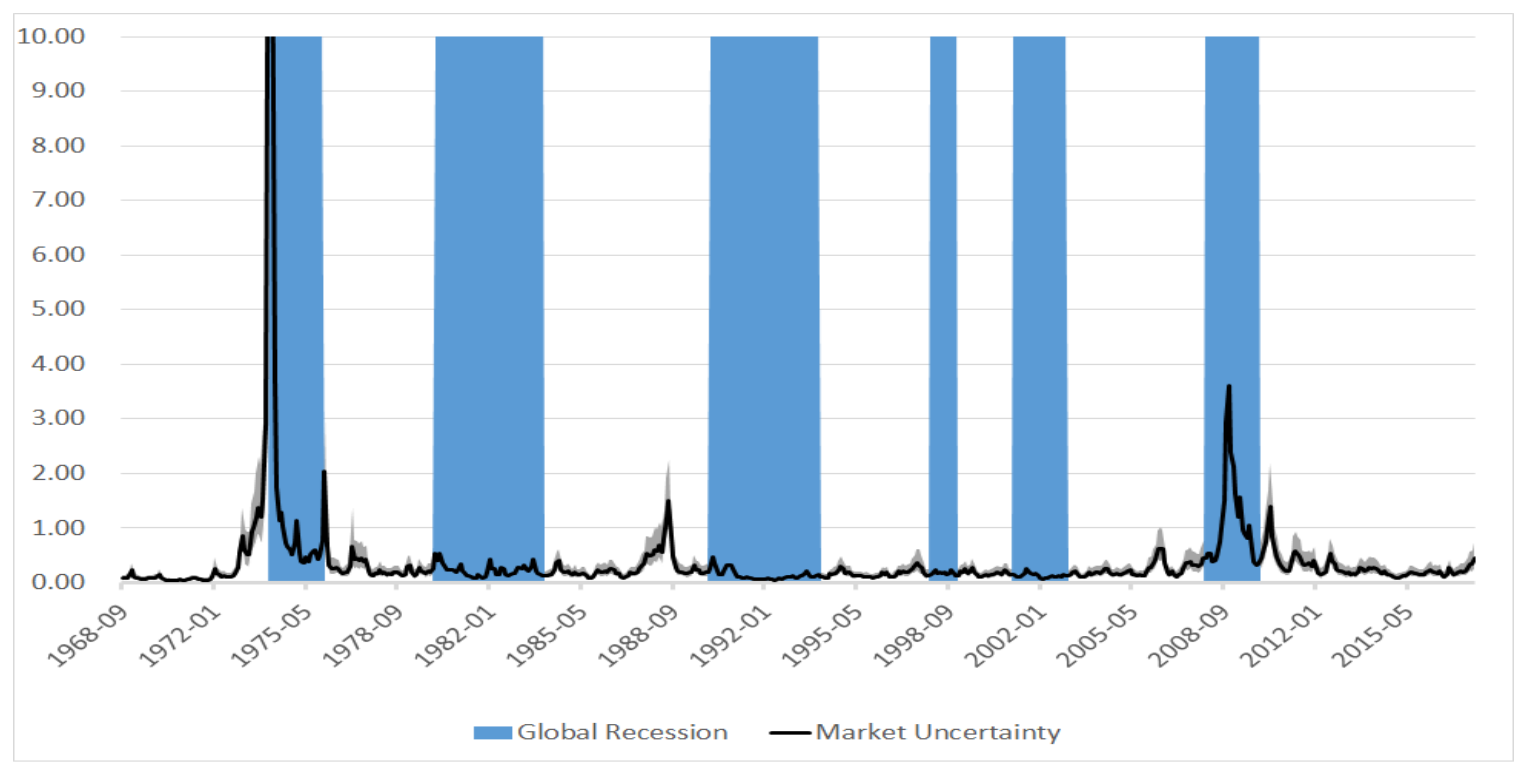

Figure 17: Market Uncertainty with 68\% Error Bands

ure 9 illustrates that the performance of the extracted static factors is heterogenous across commodities. As expected, the aggregated commodity index contains a strong commonality, in the sense that the item-specific components are smoothed by aggregation. Specifically, food index (code: IFOOD) and non-energy commodity index (code: INONFUEL) that includes agricultural item, food item, fertilizer, and metal and minerals show that $91 \%$ and $90 \%$ of the variations are explained by the first three static factors, respectively. On the other hand, $65 \%$ of the variation of the energy index (code: IENERGY) that includes coal, crude oil, and natural gas is explained by the extracted static factors. Regarding the individual item, $55 \%$ of the variation of the soybeans (code: SOYBEANS) is explained by the extracted factors. In the meanwhile, $20 \%$ of the variation of the gold (code: GOLD) is explained by those factors. However, only $3 \%$ of the variation of the chicken price (code: CHICKEN) is explained by those factors. In this light, we can expect that the factor analysis shows a good performance only for the aggregated commodity index and some of individual items.

Figure 17 shows the estimated common uncertainty from the global commodity 
markets (i.e., $\hat{\zeta}_{2 t}$ of equation (23)), which I call the market uncertainty hereafter. It clearly shows that the market uncertainty increases during the global recession periods. Specifically, the market uncertainty increases during oil crisis (1974-1975) and global financial crisis (2008-2009). In particular, the uncertainty generated by the oil crisis is much higher than that generated by the global financial crisis.

\section{Empirical Results}

In this section, I provide the estimation results from the time-varying factor-augmented VAR with stochastic volatility in mean model and take a closer look at the gold and world coffee inflation rates. To clearly describe how the ENSO affects global commodity markets, Figure 18 provides how the ENSO events create different weather patterns across large areas of the Earth. ${ }^{21}$ More importantly, the change in SST anomaly in the Niño 3.4 region affects global weather system, and therefore the global commodity markets (teleconnections) in a highly heterogenous way, although the tropical regions are mainly influenced (Hsiang and Meng, 2015). In particular, the El Niño affects agricultural productivity in the Southern Hemisphere such as Latin America, East Asia, and Australia in a negative way (Commodity Markets Outlook, 2015).

\subsection{Time-varying FAVAR with Stochastic Volatility in Mean Model}

There is a large literature that shows how the global commodity markets are closely related to the ENSO fluctuation with the small-scale VAR model. In particular, the effects of the ENSO on the global commodity markets are proven to be spatially heterogenous. Putting aside the evident fact that agricultural productivity has been affected by the ENSO level, the international energy market is also closely related to

\footnotetext{
${ }^{21}$ Source: International Research Institute for Climate and Society at Columbia University: http://iri.columbia.edu/our-expertise/climate/enso/
} 


\section{El Niño and Rainfall}

El Niño conditions in the tropical Pacific are known to shift rainfall patterns in many different parts of the world. Although they vary somewhat from one El Niño to the next, the strongest shifts remain fairly consistent in the regions and seasons shown on the map below.

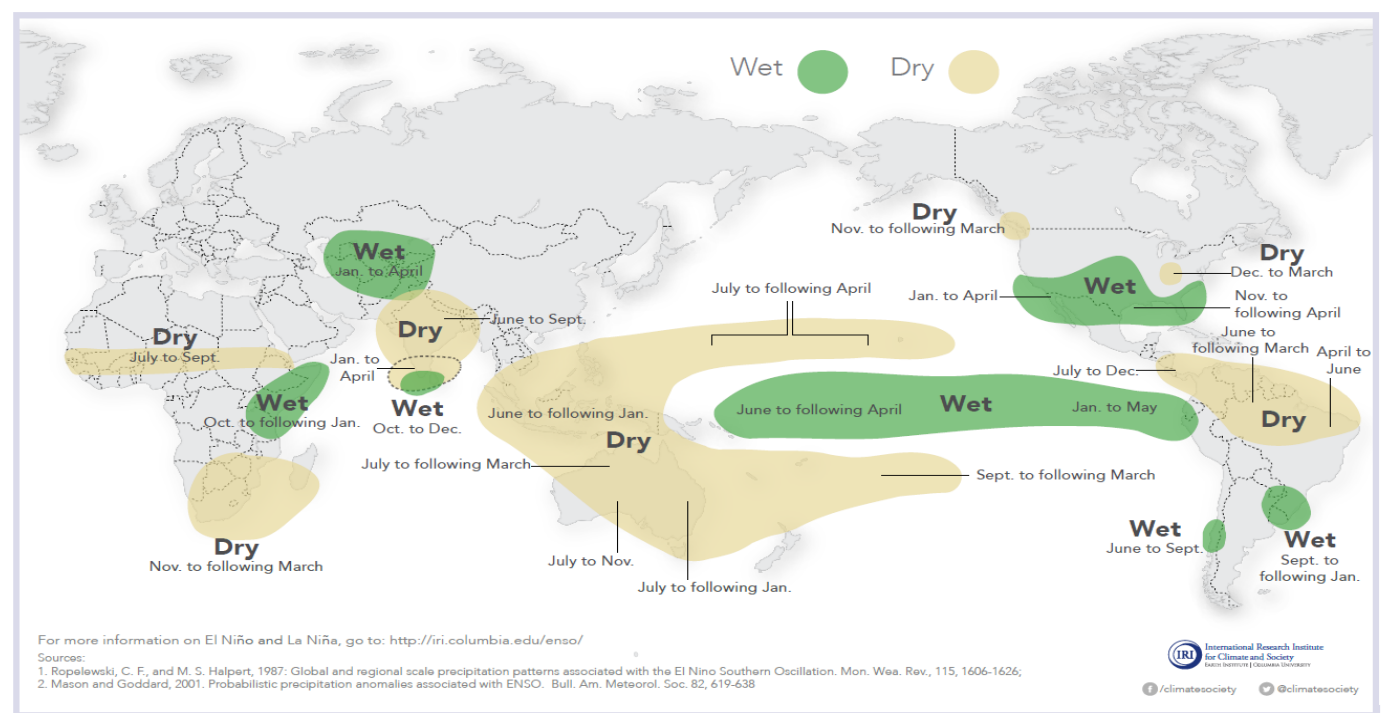

\section{La Niña and Rainfall}

La Niña conditions in the tropical Pacific are known to shift rainfall patterns in many different parts of the world. Although they vary somewhat from one La Niña to the next, the strongest shifts remain fairly consistent in the regions and seasons shown on the map below.

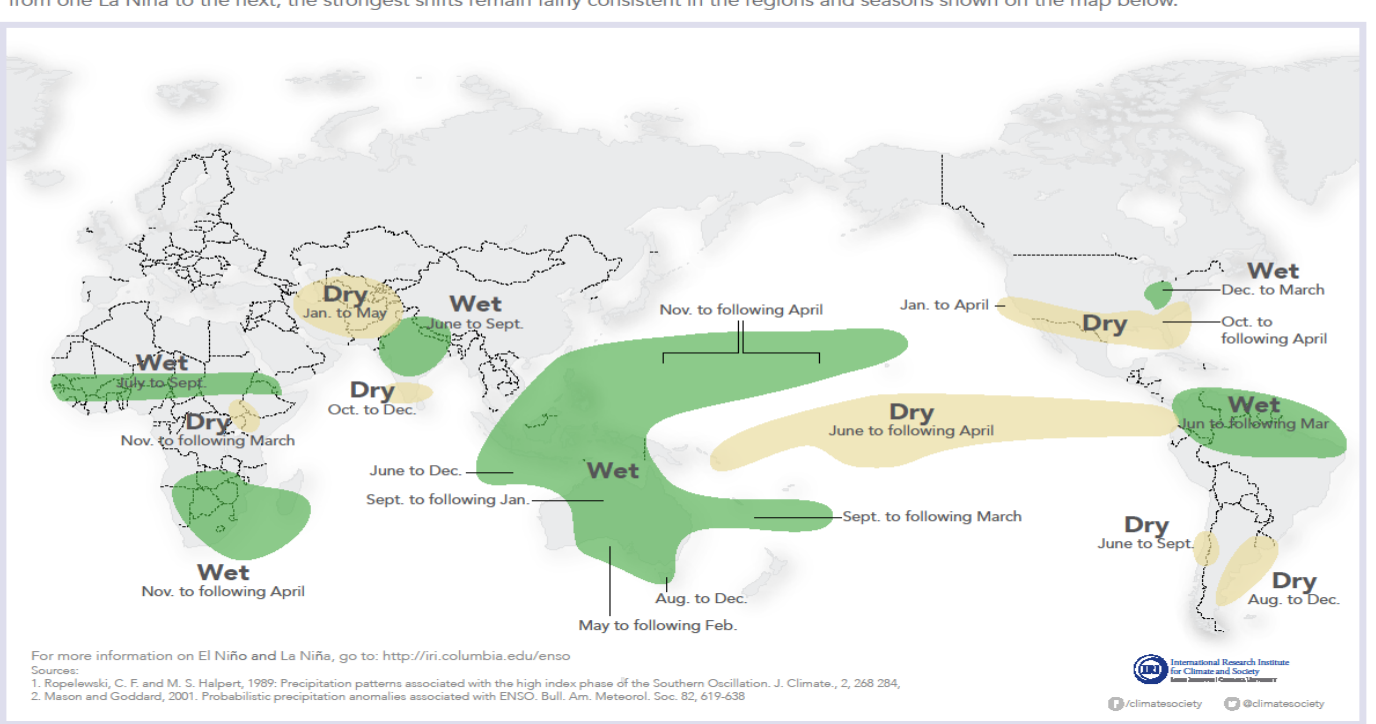

Figure 18: The spatially-heterogeneous ENSO effects 
the climate level effect. Since an El Niño event, which is a large-scale warming event in the Northern Hemisphere during December, January, and February, helps reduce world winter heating demand and fuel expenditure, a negative level shock of the SOI (i.e., El Niño) generates a deflationary pressure on the natural gas price (negative demand shock). The opposite impulse responses are expected to be generated from a La Niña event. In the meanwhile, the higher temperatures, and droughts from an El Niño event decrease hydroelectric power generation, and wind turbine electricity generation, resulting in increased demand for coal and crude oil. Moreover, since an El Niño event usually generates positive effects on the Northern Hemisphere, in which the major economies are located, the crude oil price increases to meet those increased demand.

Distinguished from the literature, I provide the time-varying effect of the climate uncertainty on the global commodity markets. More importantly, I incorporate a large amount of information into the VAR system to solve the omitted variable problem in the small-scale VAR model. It is worth noting that the climate uncertainty is defined as the one-period ahead unpredictable component of the ENSO factor, and therefore it is plausible that the climate uncertainty has the time-varying effect on the global commodity markets. Contrary to the ENSO level effect, more specifically, the uncertainty effect could be occurred when the economic agent fails to forecast the future temperature event accurately, and it is reasonable that their forecasting ability does not always hit the maximum.

The three dimensional median impulse responses are provided in Figure 19. In particular, the median impulse responses of agricultural food item (code: IFOOD, $\mathrm{R}^{2}=91 \%$ ), non-energy item (code: INONFUEL, $\mathrm{R}^{2}=90 \%$ ), energy index (code: IENERGY, $\mathrm{R}^{2}=65 \%$ ), metal \& minerals (code: IMETIN, $\mathrm{R}^{2}=60 \%$ ), maize (code: MAIZE, $\mathrm{R}^{2}=56 \%$ ), and soybeans (code: SOYBEANS, $\mathrm{R}^{2}=55 \%$ ) are provided in that Figure. I selected those items because the first three factors' explainabilities are high 

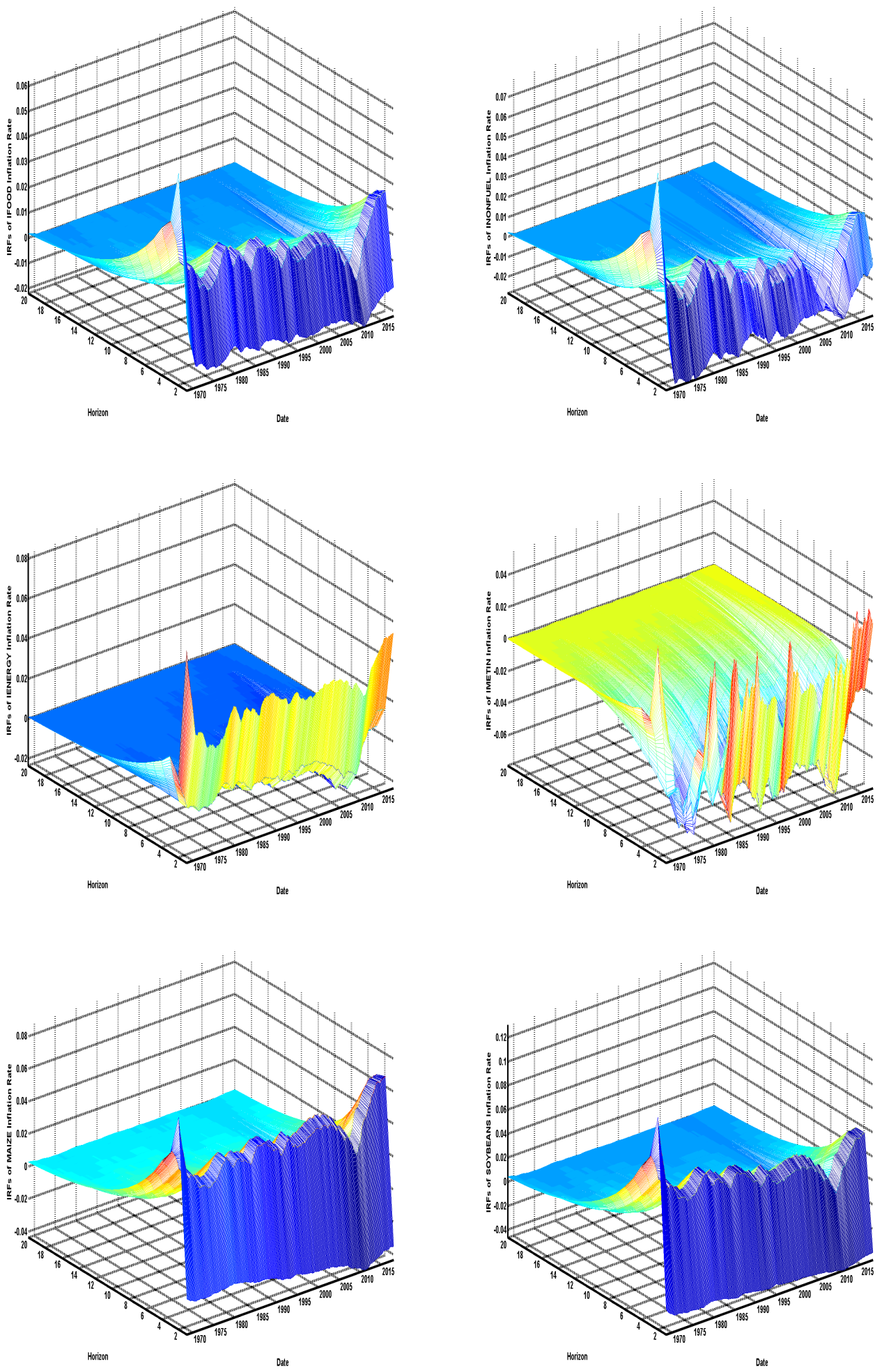

Figure 19: The selected three dimensional median impulse responses to a climate uncertainty shock 

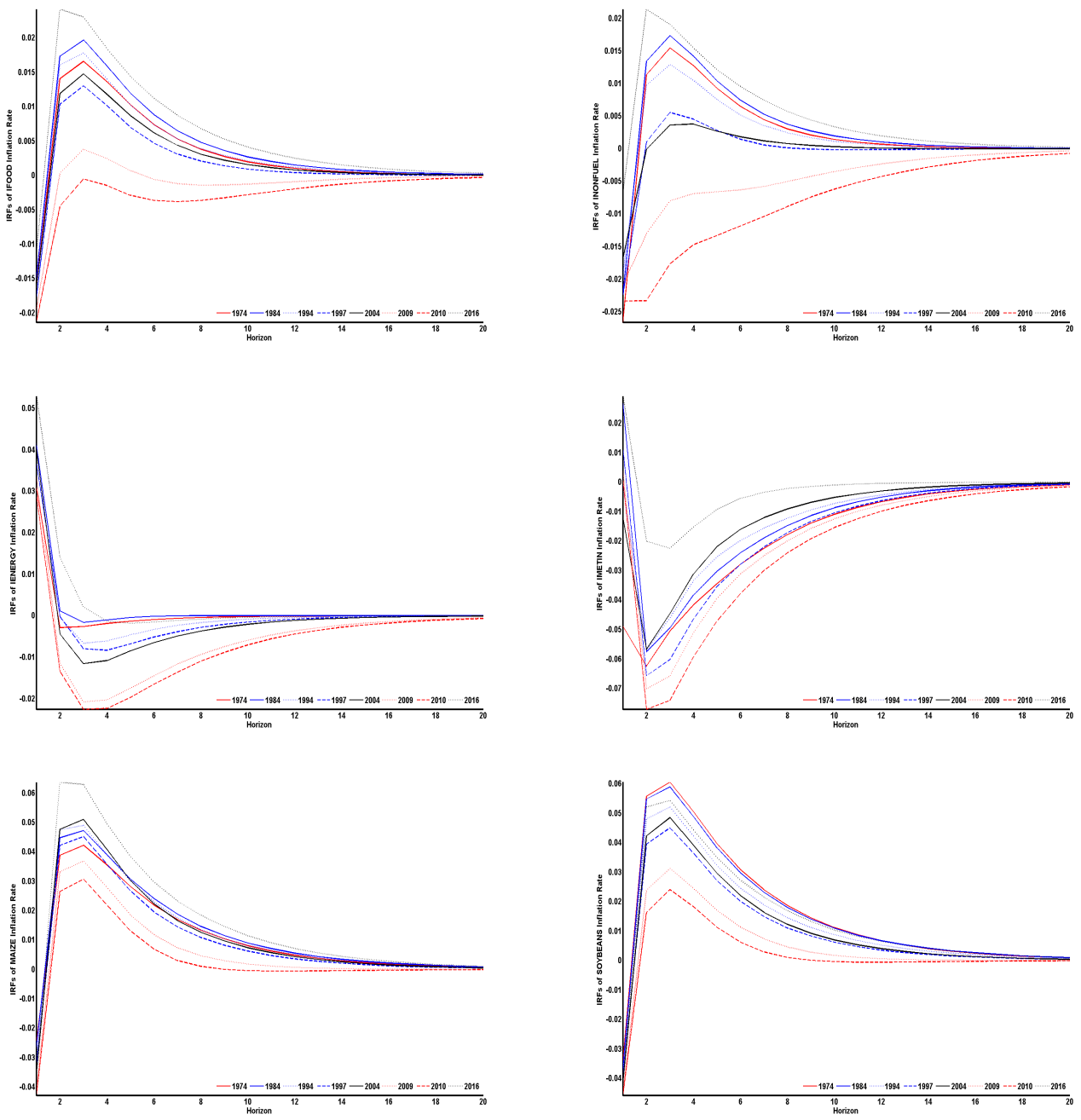

Figure 20: The yearly averaged median impulse responses to a climate uncertainty shock at selected years 
enough for the dynamic factor model to be worth considering, indicating that there may be an omitted variable problem with the small-scale VAR model (e.g., bivariate VAR model with the energy index and the SST anomaly). Since the selected three dimensional median impulse responses in Figure 19 only provide a naive insight, I also provide more detailed information from those impulse responses. Specifically, I extract the estimated time-varying impulse responses at eight years (1974, 1984, 1994, $1997,2004,2009,2010,2016)$, and then take a yearly average of the resulting twelve median impulse responses in each year. Those averaged median impulse responses are presented in Figure 20. Note that the impulse responses in the presented Figures are multiplied by 100, so that the values of impulse responses indicate the percentage change.

The effect of conditional second moment of the ENSO factor is quite different with that of the conditional first moment of the ENSO factor. When an uncertainty shock arrives at the global commodity markets, firms or crop producers delay their decision making procedure, resulting an inflationary pressure to the agricultural commodities by negative supply shock. In the meanwhile, the investors and buyers could decrease their demand for their agricultural commodities in the very short-run (i.e., negative demand shock). This implies that there would be a deflationary pressure in the very short-run, and then the climate uncertainty effect changes to an inflationary pressure.

In addition to the expected level effect, an uncertainty shock generates an inflationary pressure to the overall energy index, which is composed of coal, natural gas, and crude oil prices. ${ }^{22}$ This is because the companies or firms that use the energy increase their energy demand to hedge against the climate uncertainty (i.e., positive demand shock). In the meanwhile, there would be negative supply shock from the impact of the climate uncertainty because the energy production decision of the oil company could be delayed by the climate uncertainty.

\footnotetext{
${ }^{22}$ Specifically, the CRUDE_PETRO I employ is equally weighted average crude oil spot price of Brent, Dubai, and West Texas Intermediate.
} 
Consistent with the economic transmission mechanisms of an uncertainty shock with respect to agricultural and energy items, there are several noticeable points in the selected impulse responses in Figures 19 - 20. The effects of the climate uncertainty are generally weakly positive for the selected sample period. Notice that agricultural food and non-energy commodity prices immediately dropped and then increased at two months after a shock. As explained, this is because the reduced demand of the buyers and investors may exceed the reduced supply of the commodity producers in the very short-run. However, the climate uncertainty roughly increases the agricultural and non-energy commodity prices about $0.01-0.02 \%$, and its effect typically lasts for a year. More interestingly, the inflationary effect of the climate uncertainty gradually decreased until 2011, and then sharply increased until 2017. Notably, its effect was rapidly dropped, even to the negative sign, around the period of the global recession in 2009, implying that the climate uncertainty may be dominated by the global market uncertainty during the global recession period.

Surprisingly, the climate uncertainty generates a deflationary pressure for metal 8 mineral item. The mining activity as well as related transport infrastructure can be affected by floods or droughts (Commodity Markets Outlook, 2015). For instance, drier conditions from El Niño events during the wet season in East Asia may enhance the nickel production in the Philippines. However, an uncertainty shock generates a deflationary pressure to the prices of metal \& mineral commodities, implying that commodity buyers may reduce their demand to hedge against climate uncertainty (i.e., negative demand shock). Notice that metal \& mineral items are not a kind of cultivated crops. Therefore, there must be no negative supply shock from the impact of climate uncertainty (i.e., no inflationary pressure).

More importantly, the individual items such as maize and soybeans are more sensitive than aggregated commodity indices from the impact of the climate uncertainty. Specifically, Figure 21 illustrates the time-varying median impulse responses of maize 
and soybean inflation rates from horizon 1 to horizon 5. As expected, the impulse responses of those items at horizon 1 is negative, but insignificant. However, those impulse responses become significantly positive after two months after a shock, except for the global recession period.

As mentioned in Section 2, I generate the climate uncertainty increase by a positive one standard deviation shock on the equation (23). Therefore, it is difficult to compare the magnitude of the estimated impulse responses to a climate uncertainty shock with that to a market uncertainty shock. However, we could provide a qualitatively interesting comparison with a market uncertainty shock. Figures 22 - 23 illustrate the three dimensional median impulse responses of the selected commodities to a market uncertainty shock. Similar with the climate uncertainty, the market uncertainty generally increases the aggregated commodity prices, and its effect is particulary strong at the global recession period in 2009 .

In particular, the market uncertainty shock increases the energy price by about $0.12 \%$ during the global recession period, which is reasonable because the market uncertainty also generates the negative supply shock (Elder and Serletis 2009, 2010; Jo 2014). However, the market uncertainty decreases individual agricultural food prices such as maize and soybeans. This is because the global financial market turmoil decreases the demand of buyers and investors for individual food items, more than the decreased supply of the commodity producers in the short run.

\subsection{Testing for a Safe Haven Asset Hypothesis}

The gold is often considered as a safe haven asset in times of crisis. People purchase gold for several purposes. Among them, the most important function is that it can be an asset that provides a hedging tool against inflation, change in exchange rate, or economic uncertainty. Put differently, people consider it as a kind of insurance against negative economic events. When a negative economic event happens, investors may 

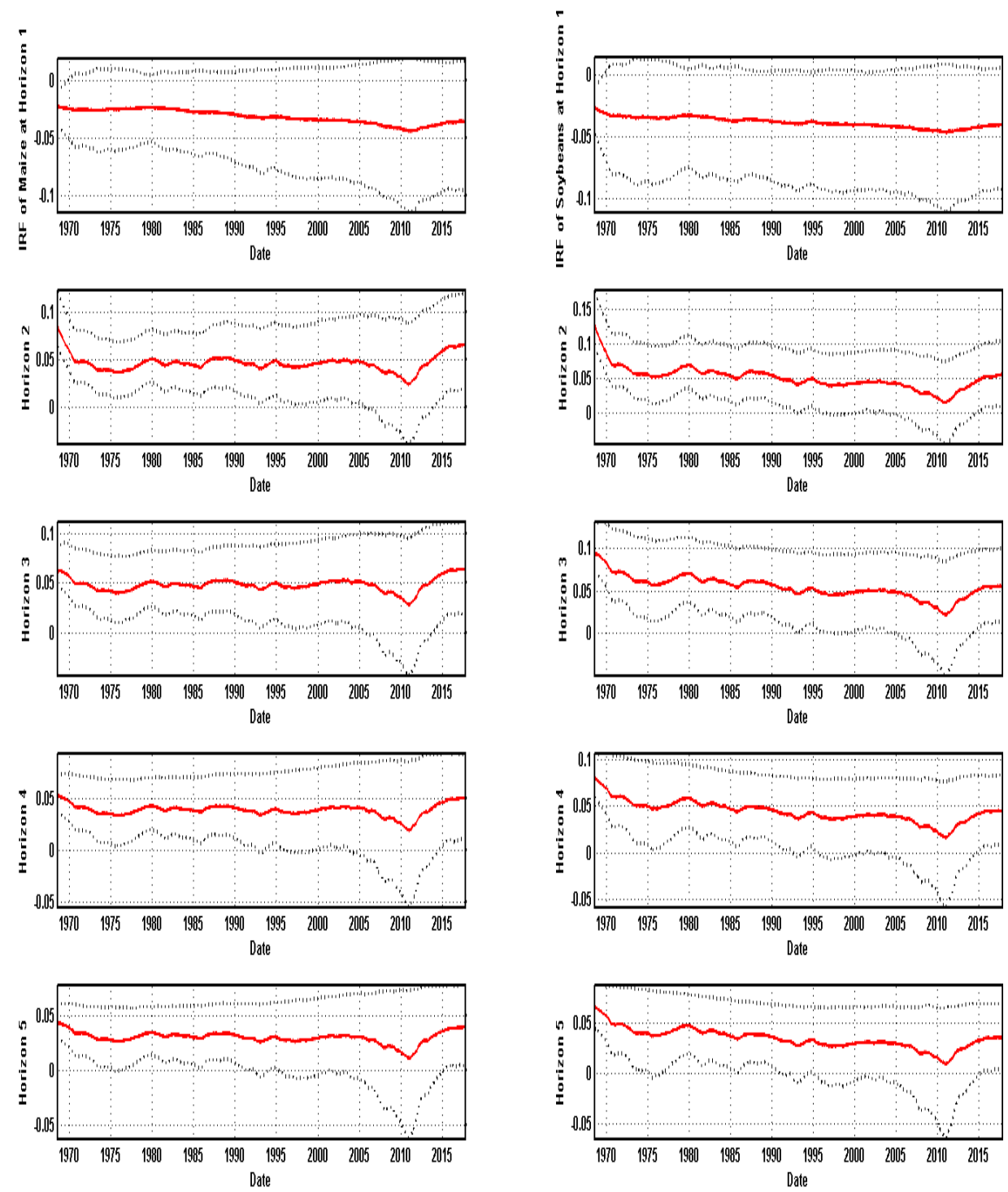

Figure 21: The time-varying median impulse responses of Maize (left column) and Soybean (right column) inflation rate to a climate uncertainty shock at horizons 1-5 with $68 \%$ error bands 

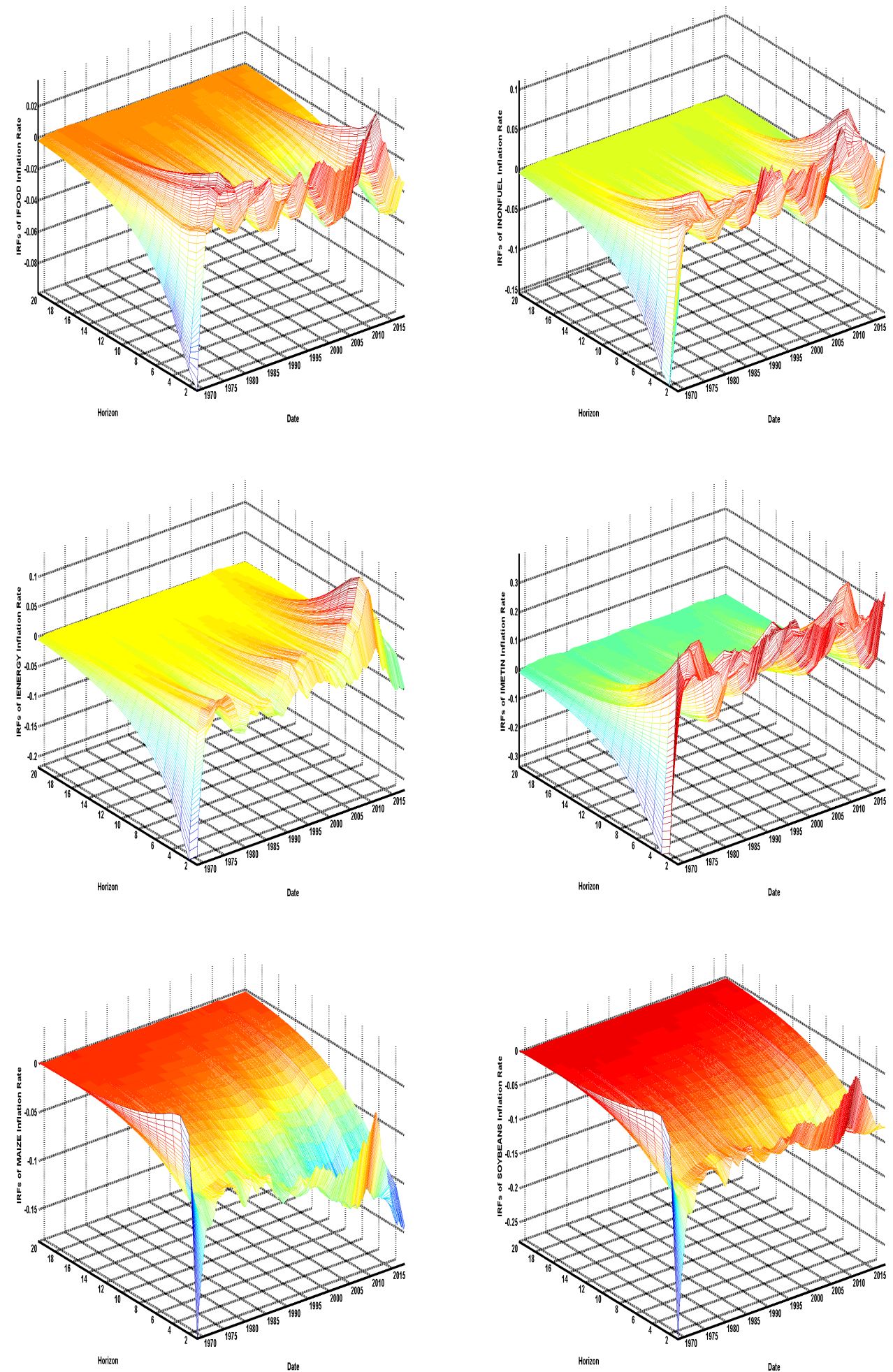

Figure 22: The selected median impulse responses to a market uncertainty shock 

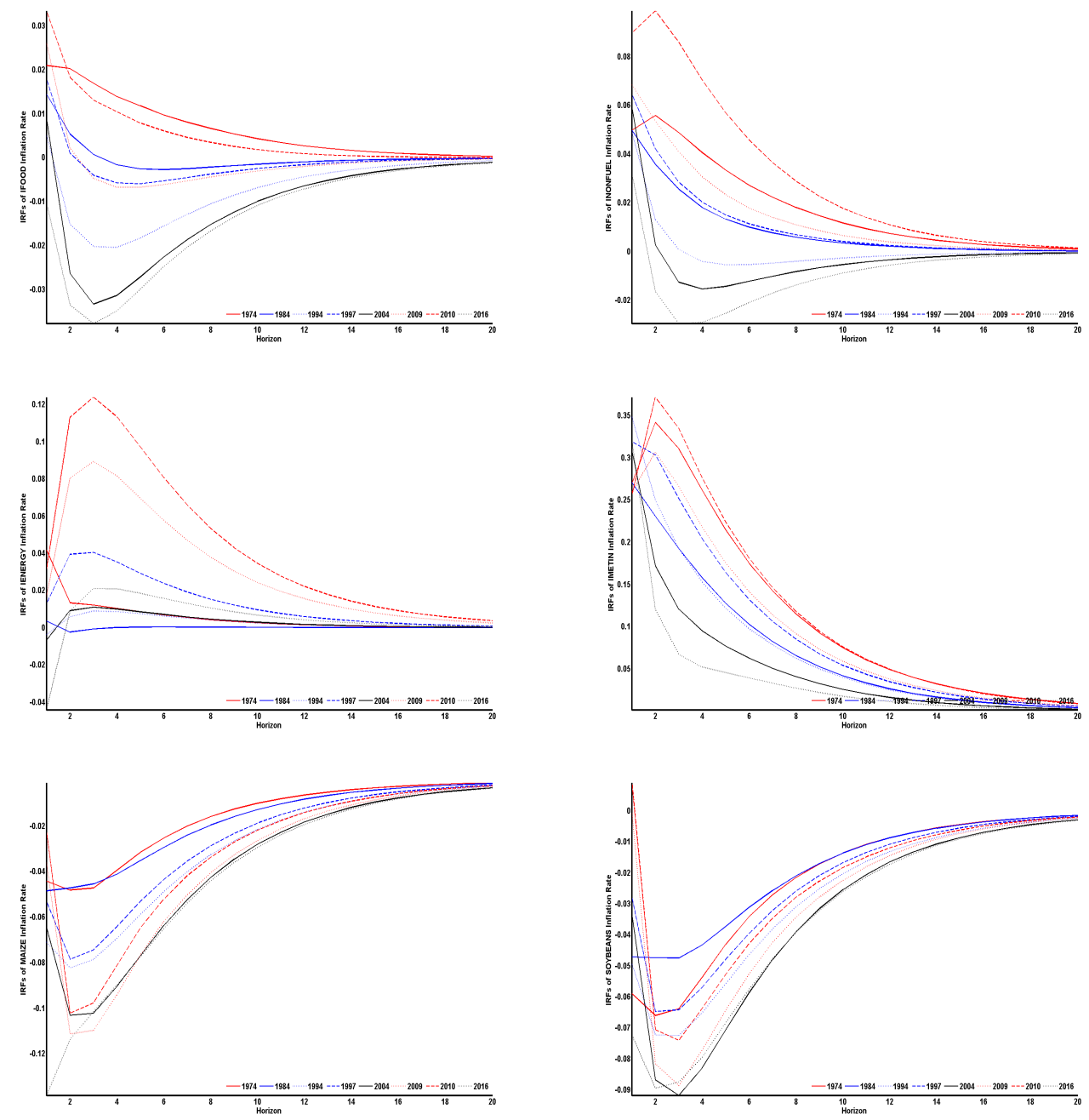

Figure 23: The yearly averaged median impulse responses to a market uncertainty shock at selected years 

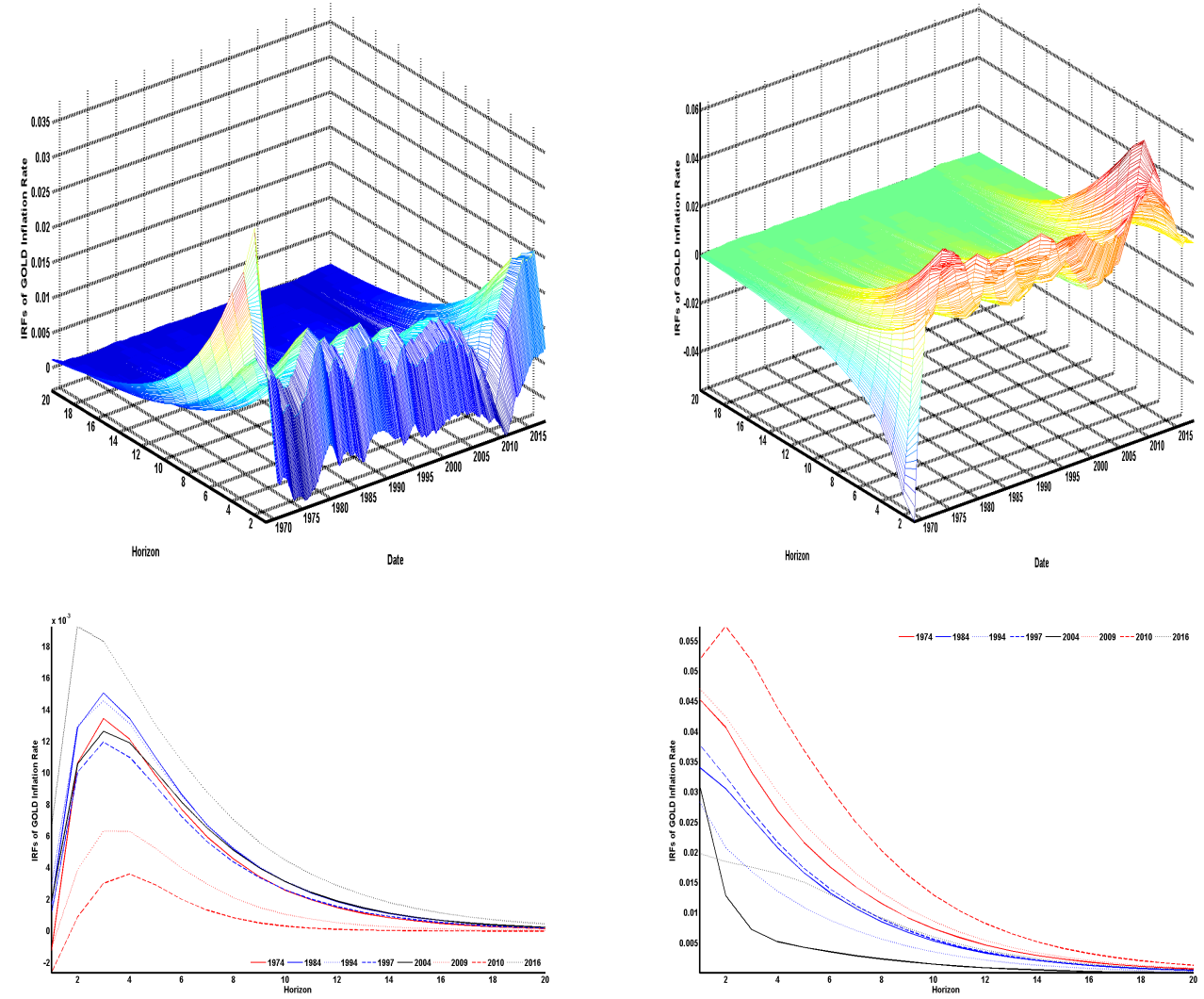

Figure 24: The median impulse responses of Gold inflation rate to a climate uncertainty shock (left column) and those to a market uncertainty shock (right column) 
want to spend their funds to buy gold, increasing its price via increased demand. In this vein, testing whether the gold serves as a safe haven asset during the times of climate uncertainty is worth investigating.

Figure 24 illustrates the three dimensional median impulse responses and their two dimensional slices at selected years. Similar with the previous cases, both the climate and market uncertainties generate an inflationary pressure for the sample period. Unlike the market uncertainty effect, however, the climate uncertainty effect was disappeared during the global recession period. This is because the global market uncertainty could dominate the climate uncertainty in that period. Moreover, the magnitudes of the impulse responses to a climate uncertainty shock are very small in an insignificant way, compared with those to a market uncertainty shock, implying that the global market uncertainty may have a bigger role than the climate uncertainty to explain the safe haven asset hypothesis.

\subsection{Application to World Coffee Inflation Rates}

Ubilava (2012) provided an interesting empirical work on the relationship between the ENSO and world coffee prices using time-varying smoothed transition vector error correction model. Considering the geographical areas of production of two world coffee, it is intuitive that the ENSO generates opposite effects on Robusta and Arabica coffee prices. Specifically, two geographical areas - Southeast Asia (Vietnam and Indonesia) where Robusta coffee is predominantly produced, and Central and South American countries (Brazil and Colombia) where Arabica Milds is predominantly produced - are affected by the ENSO in an opposite way. Put differently, the El Niño events create unfavorable weather condition in Southeast Asia and favorable weather condition in Central and South America, which result in increased Robusta coffee price and decreased Arabica coffee price. The opposite price changes have occurred during La Niña events. Since Arabica coffee is considered as higher quality than 
Robusta coffee, the El Niño events decrease the difference between two coffee prices.

With this paper, I investigate the effect of climate uncertainty on world coffee inflation rates whereas Ubilava (2012) only provides positive and negative level effects. Note that if the uncertainty effect is statistically significant, then the model employed by Ubilava (2012) suffers from the omitted variable problem and therefore, the level effect must be amplified or attenuated based on sign of the level effect. In Figure 25, I provide the estimated three dimensional median impulse responses of Arabica and Robusta coffee inflation rates to an uncertainty shock. Note that the first three principal components explain $11 \%$ of the total variation of Arabica coffee inflation rate, and $12 \%$ of that of Robusta coffee inflation rate, implying that the only onetenth of the total variations of two coffee inflation rates has been explained by three factors.

As expected, the climate uncertainty generates a negative supply shock, resulting increased Arabica and Robusta coffee prices for the sample period. On the contrary, the global market uncertainty generates a negative demand shock, resulting decreased Arabica and Robusta coffee prices for the sample period. Note that Robusta coffee production is more concentrated in the ENSO affected region, while Arabica coffee production is spread to different regions of America and Africa. Moreover, Arabica coffee maintains higher quality with big four customers (Kraft, Nestle, Procter \& Gamble, and Sara Lee), implying that Arabica coffee producers are more insensitive to an unexpected temperature event. However, the estimation results in Figures 25-26 show that the climate uncertainty effects are roughly similar. Rather, Arabica coffee price is more sensitive before 1975 and after 2011, implying that world coffee price application may not be a good choice to show how the uncertainty effect works. 

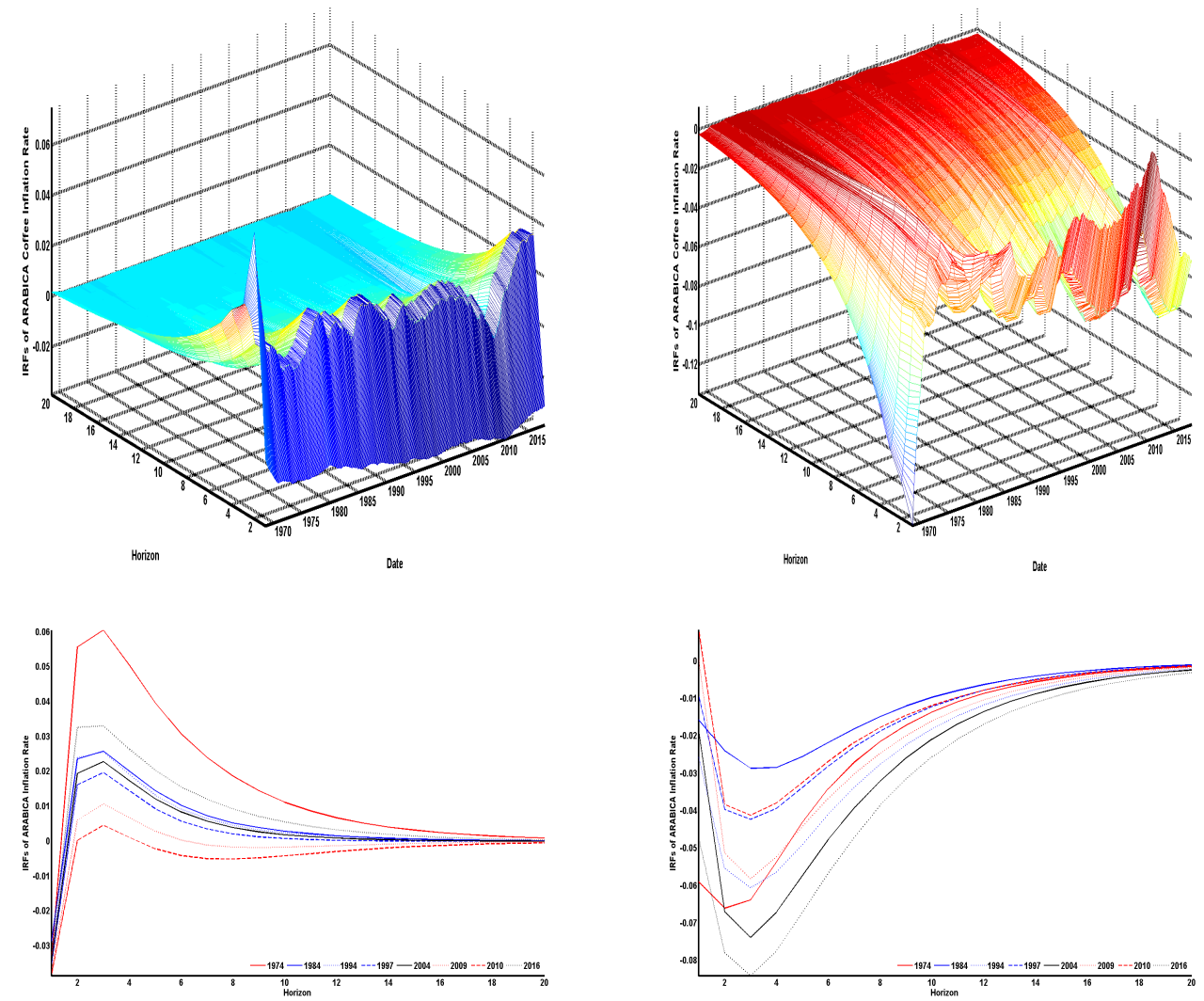

Figure 25: The median impulse responses of Arabica coffee inflation rate to a climate uncertainty shock (left column) and those to a market uncertainty shock (right column) 

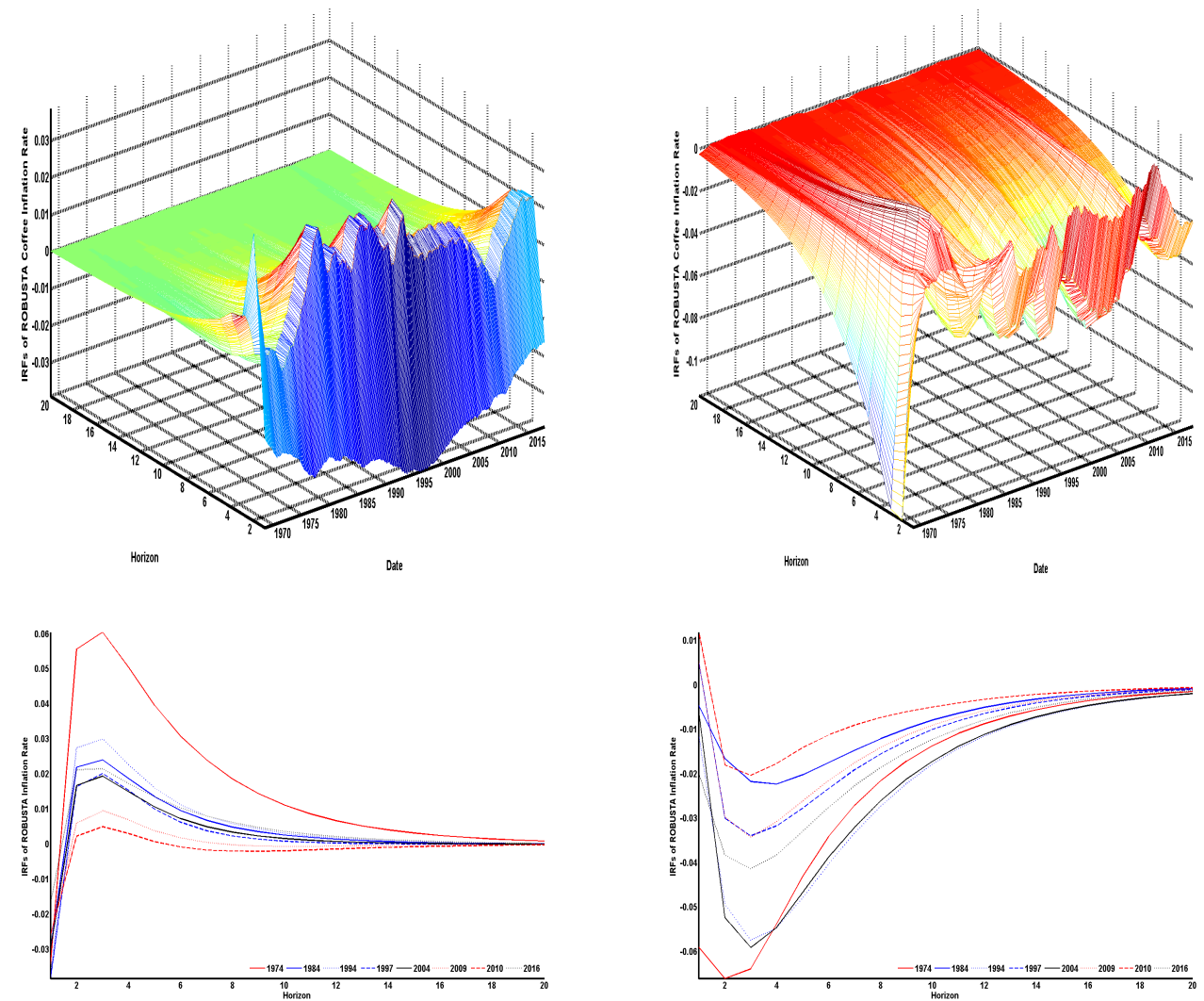

Figure 26: The median impulse responses of Robusta coffee inflation rate to a climate uncertainty shock (left column) and those to a market uncertainty shock (right column) 


\section{Concluding Remarks}

This paper provides a statistical analysis of the climate impact on the global commodity markets. More precisely, the empirical analyses show the effects of the conditional second moments of the El Niño events on the global commodity prices. To do so, I utilize the time-varying factor-augmented VAR with stochastic volatility in mean model. The estimation is implemented via one-step Bayesian procedure. By incorporating a parsimoniously sophisticated factor structure with volatility in mean effect, the model analyzed in this paper not only frees from possible omitted variable bias but also maintains the efficiency of the estimators. The estimation results indicate that the climate uncertainty generally increases global commodity prices. Considering that the climate uncertainty creates a negative supply shock on global commodity markets, the estimation results are appealing to the literature.

Precisely speaking, the ENSO dynamics is better represented with three states (El Niño state, La Niña state, and neutral state), and their regime switching speed is known to be asymmetrical. This suggests that the three-state endogenous regime switching in mean model with $\operatorname{AR}(2)$ latent factor dynamics could be a better approach to extract more reasonable ENSO factor. Moreover, as I employ the monthly frequency data, the optimal autoregressive order may be higher than four lags that this paper chose. In addition, the ENSO level effect, which takes account of the uncertainty effect, has to be re-examined. I leave these tasks for my future research.

Furthermore, as noted by Gross (2017), future markets have several advantages over spot markets in analyzing the comovement of the global commodity markets. First, future commodity prices are more responsive to new information than spot commodity prices. Second, the effects of speculation are better explained by future markets. However, a relatively short-time span and a small number of available commodities may be an obstacle to extend my analysis to the future markets. I also leave it for my future research. 


\section{References}

Alquist R, Coibion O. 2014. Commodity price comovement and global economic activity. NBER Working Paper 20003.

Andrle M, Bruha, J, Solmaz, S. 2016. On the sources of business cycles: implications for DSGE models. CNB Working Paper.

Asaia M, McAleer M. 2009. The structure of dynamic correlations in multivariate stochastic volatility models. Journal of Econometrics 150: 182-192.

Bai J, Ng S. 2002. Determining the number of factors in approximate factor models. Econometrica 70: 191-223.

Bai J. 2003. Inferential theory for factor models of large dimensions. Econometrica 71: $135-171$.

Belviso F, Milani F. 2006. Structural factor-augmented VARs (SFAVARs) and the effects of monetary policy. Topics in Macroeconomics 6: 1443-1443.

Bernanke B. 1983. Irreversibility, uncertainty, and cyclical Investment. Quarterly Journal of Economics 98: 85-106.

Bernanke B, Boivin J. 2003. Monetary policy in a data-rich environment. Journal of Monetary Economics 50: 525-546.

Bernanke B, Boivin J, Eliasz P. 2005. Measuring the effects of monetary policy: a factor-augmented vector autoregressive (FAVAR) Approach. The Quarterly Journal of Economics 120: 387-422.

Bjerknes J. 1969. Atmospheric teleconnections from the equatorial pacific. Journal of Physical Oceanography 97: 163-172.

Bloom N. 2009. The impact of uncertainty shocks. Econometrica 77: 623-685. 
Boer GJ, Yu B. 2003. Climate sensitivity and response. Climate Dynamics 20: 415-429.

Brillinger DR. 1981. Time series: data analysis and theory. Holden-Day: San Francisco.

Brunner AD. 2002. El Niño and world primary commodity prices: warm water or hot air? Review of Economics and Statistics 84: 176-183.

Burke M., Hsiang SM, Miguel E. 2015. Global non-linear effect of temperature on economic production. NATURE 527: 235-239.

Byrne JP, Fazio G, Fiess, N. 2013. Primary commodity prices: co-movements, common factors and fundamentals. Journal of Development Economics 101: $16-26$.

Campbell SD, Diebold FX. 2005. Weather forecasting for weather derivatives. Journal of the American Statistical Association 100: 6-16.

Carriero A, Clark TE, Marcellino M. 2015. Common drifting volatility in large Bayesian VARs. Journal of Business and Economic Statistics 34: 375-390.

Carter CK, Kohn R. 1994. On gibbs sampling for state space models. Biometrika 81: $541-553$.

Cashin P, Mohaddes K, Raissi M. 2017. Fair weather or foul? the macroeconomic effects of El Niño. Journal of International Economics 106: 37-54.

Chang Y, Choi Y, Park JY. 2017. A new approach to model regime switching. Journal of Econometrics 196: 127-143.

Chang Y, Kwak B. 2017. U.S. monetary-fiscal regime changes in the presence of endogenous feedback in policy rules. Working paper. 
Chen SL, Jackson JD, Kim H, Resiandini P. 2014. What drives commodity prices? American Journal of Agricultural Economics 96: 1455-1468.

Chiaie SD, Ferrara L, Giannone D. 2017. Common factors of commodity prices. Working paper.

Cogley T, Sargent TJ. 2005. Drift and volatilities: monetary policies and outcomes in the post WWII U.S. Review of Economic Dynamics 8: 262-302.

Delnegro M, Otrok C. 2005. Dynamic factor models with time-varying parameters. Mimeo, Federal Reserve Bank of Atlanta.

Elder J. 2004. Another perspective on the effects of inflation uncertainty. Journal of Money, Credit and Banking 36: 911-28.

Elder J, Serletis A. 2009. Oil price uncertainty in Canada. Energy Economics 31: 852-56.

Elder J, Serletis A. 2010. Oil price uncertainty. Journal of Money, Credit and Banking 42: 1137-59.

Estrada F, Perron P, Martínez-López, B. 2013. Statistically derived contributions of diverse human influences to twentieth-century temperature changes. Nature Geoscience 6: 1050-1055.

Favero CA, Marcellino M, Neglia F. 2005. Principal components at work: the empirical analysis of monetary policy with large data sets. Journal of Applied Econometrics 20: 603-320.

Forni M, Hallin M, Lippi M, Reichlin L. 2000. The generalized factor model: identification and estimation. The Review of Economics and Statistics 82: 540-554. 
Forni M, Hallin M, Lippi M, Reichlin L. 2003. Do financial variables help forecasting inflation and real activity in the euro area? Journal of Monetary Economics 50: $1243-1255$.

Forni M, Hallin M, Lippi M, Reichlin L. 2005. The generalized dynamic factor model: one-sided estimation and forecasting. Journal of the American Statistical Association 100: 830-840.

Goddard L, Mason SJ, Zebiak SE, Ropelewski CF, Basher R, Cane MA. 2001. Current approaches to seasonal to interannual climate predictions. International Journal of Climatology 21: 1111-1152.

Gross C. 2017. Examining the common dynamics of commodity futures prices. Working paper.

Hamilton JD. 2018. Why you should never use the Hodrick-Prescott filter. Review of Economics and Statistics, forthcoming.

Hsiang SM, Meng KC. 2015. Tropical economics. American Economic Review: Papers \& Proceedings 105: 257-261.

Jacquier E, Polson NG, Rossi PE. 1994. Bayesian analysis of stochastic volatility models. Journal of Business and Economic Statistics 12: 371-89.

Jo S. 2014. The effects of oil price uncertainty on global real economic activity. Journal of Money, Credit and Banking 46: 1113-35.

Jurado K, Ludvigson SC, Ng S. 2015. Measuring uncertainty. American Economic Review 105: 1177-1216.

Kaufmann RK, Kauppi H, Mann ML, Stock JH. 2013. Does temperature contain a stochastic trend: linking statistical results to physical mechanisms. Climatic Change 118: 729-743. 
Kaufmann RK, Kauppi H, Stock JH. 2006a. Emissions, concentrations and temperature: a time series analysis. Climatic Change 77: 249-278.

Kaufmann RK, Kauppi H, Stock JH. 2006b. The relationship between radiative forcing and temperature: what do statistical analyses of the instrumental temperature record measure? Climatic Change 77: 279-289.

Kaufmann RK, Kauppi H, Stock JH. 2010. Does temperature contain a stochastic trend? evaluating conflicting statistical results. Climatic Change 101: 395-405.

Kilian L. 1998. Small-sample confidence intervals for impulse response functions. The Review of Economics and Statistics 80: 218-230.

Kim C, Nelson CR. 1999. State-space models with regime switching. Cambridge, MA: MIT Press.

Kim H, Lee HI, Park JY, Yeo H. 2009. Macroeconomic uncertainty and asset prices: a stochastic volatility model. Working Paper.

Kim S, Shephard N, Chib S. 1998. Stochastic volatility: likelihood inference and comparison with ARCH models. Review of Economic Studies, 65: 361-93.

Koop G, Pesaran MH, Potter SM. 1996. Impulse response analysis in nonlinear multivariate models. Journal of Econometrics 74: 119-147.

Koop G, Potter SM. 2011. Time varying (VARs) with inequality restrictions. Journal of Economic Dynamics and Control 35: 1126-1138.

Koopman SJ, Uspensky EH 2002. The stochastic volatility in mean model: empirical evidence from international stock markets. Journal of Applied Econometrics 17: 667-689.

Laosuthi T, Selover DD. 2007. Does El Niño affect business cycles? Eastern Economic Journal 33: 21-42. 
Latif M, Keenlyside NS. 2009. El Niño/Southern Oscillation response to global warming. PNAS 106: 20578-20583.

Liu P, Mumtaz H, Theophilopoulou A. 2014. The transmission of international shocks to the UK. Estimates based on a time-varying factor augmented VAR. Journal of International Money and Finance 46: 1-15.

Miller JI. 2017. Local climate sensitivity: a statistical approach for a spatially heterogeneous planet. University of Missouri, Department of Economics, Working Paper.

Miller, JI, Nam K. 2017. Dating hiatuses: a statistical approach to the recent pause in global warming - and the next one. University of Missouri, Department of Economics, Working Paper.

Nam K. 2018. Nonlinear cointegrating regression of Earth's surface mean temperature anomalies on total radiative forcing, Working Paper.

Mumtaz H. 2010. Evolving UK macroeconomic dynamics: a time-varying factor augmented VAR. Working paper.

Mumtaz H, Surico P. 2009. The transmission of international shocks: a factoraugmented VAR approach. Journal of Money, Credit and Banking 41: 71-100.

Mumtaz H, Theodoridis K. 2018. The changing transmission of uncertainty shocks in the U.S. Journal of Business and Economic Statistics 36: 239-252.

Mumtaz H, Zanetti F. 2013. The impact of the volatility of monetary policy shocks. Journal of Money, Credit and Banking 45: 535-558.

Pindyck RS, Rotemberg JJ. 1990. The excess co-movement of commodity prices. The Economic Journal 100: 1173-1189. 
Poppick A, Moyer EJ, Stein ML. 2017. Estimating trends in the global mean temperature record. Advances in Statistical Climate, Meteorolology, and Oceanography 3: $33-53$.

Primiceri GE. 2005. Time varying structural vector autoregressions and monetary policy. Review of Economic Studies 72: 821-852.

Ravn MO, Uhlig H. 2002. Notes on adjusting the Hodrick-Prescott filter for the frequency of observations. The Review of Economics and Statistics 84: 371380.

Schlenker W, Roberts MJ. 2009. Nonlinear temperature effects indicate severe damages to U.S. crop yields under climate change. PNAS 106: 15594-15598.

Shin M, Zhong M. 2018. A new approach to identifying the real effects of uncertainty shocks. Journal of Business and Economic Statistics, forthcoming.

Stock JH, Watson MW. 2002. Macroeconomic forecasting using diffusion indexes. Journal of Business and Economic Statistics 20: 147-162.

Ubilava D. 2012. El Niño, La Niña, and world coffee price dynamics. Agricultural Economics 43: 17-26.

Ubilava D. 2014. El Niño Southern Oscillation and the fishmealsoya bean meal price ratio: regime-dependent dynamics revisited. European Review of Agricultural Economics 41: 583-604.

Ubilava D. 2017. The role of El Niño Southern Oscillation in commodity price movement and predictability. American Journal of Agricultural Economics, forthcoming.

Ubilava D, Helmers CG. 2013. Forecasting ENSO with a smooth transition autoregressive model. Environmental Modelling \& Software 40: 181-190. 
West KD, Wong KF. 2014. A factor model for co-movements of commodity prices. Journal of International Money and Finance 42: 289-309.

World Bank Group. 2015. Commodity markets outlook: understanding El Niño. A World Bank Quarterly Report. 


\section{Appendices}

\section{Optimization Procedures}

To achieve a global maximizer, I utilize both profile likelihood surface algorithm and Global and Multistart search algorithm, which are provided in Matlab software, with tolerance level $10^{-10}$ for both changes in parameter and function value. Specifically, Global and Multistart search algorithm exploits fmincon optimization function from multiple start points. The Global Search algorithm uses a scatter-search mechanism for generating start points, while Multistart algorithm uses uniformly distributed start points within constraints. In my procedure, Global Search algorithm analyzes 10,000 start points and rejects those points that are unlikely to improve the best local minimum found. On the other hand, MultiStart algorithm runs 1,000 start points in parallel, distributing start points to multiple processors for a local solution. Finally, I choose an algorithm that provides a greater log-likelihood value. The asymptotic covariance matrix is obtained by the inverse of Fisher's information matrix from reconducting optimization at the best estimates with small deviations that ensure the same estimates I obtained. The detailed procedure for the profile likelihood surface algorithm is in Chang et al. (2017).

Precisely speaking, we have four optimization schemes as follows.

1 Without generating profile likelihood surface and G/M search algorithms, directly estimate the whole parameters, $\underline{\mu}, \bar{\mu}, \tau, \sigma, \gamma, \alpha, \rho$.

2 With generating profile likelihood surface and without G/M search algorithms, sequentially estimate the parameters, $\underline{\mu}, \bar{\mu}, \tau, \sigma, \gamma$ and then $\underline{\mu}, \bar{\mu}, \tau, \sigma, \gamma, \alpha, \rho$.

3 Without generating profile likelihood surface and with G/M search algorithms, directly estimate the whole parameters, $\underline{\mu}, \bar{\mu}, \tau, \sigma, \gamma, \alpha, \rho$. 
4 With generating profile likelihood surface and G/M search algorithms, sequentially estimate the parameters, $\underline{\mu}, \bar{\mu}, \tau, \sigma, \gamma$ and then $\underline{\mu}, \bar{\mu}, \tau, \sigma, \gamma, \alpha, \rho$.

Obviously, the order of computing time is $(1)<(2)<(3)<(4)$. Practically, the scheme (1) takes less than 10 minutes. Based on my experiences, estimates from the scheme (1) are not reliable. But, estimates from the schemes (2), (3), and (4) are reliable. In this context, I use the scheme (3) to obtain a global maximizer.

Since the calculated standard errors depend on initial points when using MLE procedure, estimated standard errors from schemes, (1)-(4) are all different. For instance, suppose that we get best estimates from the scheme (2) or (3). Then, the initial point of the scheme (1) is the best estimates that we obtained with small deviations, whereas the initial point of the scheme (2) is a point that maximizes profile likelihood surface (best estimates with small deviations under fixed $\alpha$ and $\rho$ ). However, it is worth noting that whatever scheme we use, the parameter estimates are invariant and therefore, extracted latent factors are invariant. The standard errors reported in Table 3 are calculated from scheme (3).

\section{Estimating the Climate Uncertainty}

Based on the temperature modeling in Campbell and Diebold (2005), I estimate the deseasonalized sea surface temperature (SST) anomaly data by the model, given by

$$
\begin{aligned}
S S T_{t}= & \sum_{i=0}^{I} \alpha_{i} t^{i}+\sum_{j_{1}=1}^{J_{1}}\left(\beta_{1 j_{1}} \cos 2 j_{1} \pi\left(\frac{t}{T}\right)+\beta_{2 j_{1}} \sin 2 j_{1} \pi\left(\frac{t}{T}\right)\right)+\sum_{p_{1}=1}^{P_{1}} \gamma_{p_{1}} S S T_{t-p_{1}} \\
& +\sigma_{t} \varepsilon_{1 t} \\
\log \sigma_{t} & =\sum_{j_{2}=1}^{J_{2}}\left(\beta_{1 j_{2}} \cos 2 j_{2} \pi\left(\frac{t}{T}\right)+\beta_{2 j_{2}} \sin 2 j_{2} \pi\left(\frac{t}{T}\right)\right)+\sum_{p_{2}=1}^{P_{2}} \gamma_{p_{2}} \log \sigma_{t-p_{2}}+\varepsilon_{2 t}
\end{aligned}
$$


with $\varepsilon_{1 t} \sim$ i.i.d.N $(0,1)$ and $\varepsilon_{2 t} \sim$ i.i.d.N $(0, Q)$. Note that the concept of climate uncertainty defined by equation (31) is different with that I defined in Sections 2 and 3. Assume that the economic agents forecast the future temperature based on the past temperature information. Specifically, the economic agents recognize that the temperature dynamics can be decomposed into three parts; time trend, cyclicality, and high-frequency cycle. The time-trend and periodicity are forecast by the deterministic time trend and a fourier series, respectively. Since the high-frequency cycle shows stochastic movements, however, the economic agents use the past temperature information using autoregressive lags. In this vein, the climate uncertainty stems from two sources. One is the prediction error generated by using the deterministic information. The another is the prediction error generated by using stochastic autoregressive terms. Note that the climate uncertainty this paper defines only comes from the former, but with two-state regime-switching process.

Based on the estimation procedure in Kim and Nelson (1999) and Primiceri (2005), I define

$$
\begin{aligned}
y_{t}^{*} \equiv & S S T_{t}-\sum_{i=0}^{I} \alpha_{i} t^{i}-\sum_{j_{1}=1}^{J_{1}}\left(\beta_{1 j_{1}} \cos 2 j_{1} \pi\left(\frac{t}{T}\right)+\beta_{2 j_{1}} \sin 2 j_{1} \pi\left(\frac{t}{T}\right)\right)-\sum_{p_{1}=1}^{P_{1}} \gamma_{p_{1}} S S T_{t-p_{1}} \\
& =\sigma_{t} \varepsilon_{1 t}
\end{aligned}
$$

then, we can rewrite it as

$$
y_{t}^{* 2} \equiv \sigma_{t}^{2} \varepsilon_{1 t}^{2}
$$

Let's define

$$
y_{t}^{* *}=\log \left[y_{t}^{* 2}+\bar{c}\right], \quad e_{t}=\log \left(\varepsilon_{1 t}^{2}\right), h_{t}=\log \left(\sigma_{t}\right)
$$


where $\bar{c}$ is a offset constant (1e-6). Then, the measurement and transition equations can expressed as.

$$
\begin{aligned}
y_{t}^{* *} & =2 h_{t}+e_{t} \\
h_{t} & =\sum_{j_{2}=1}^{J_{2}}\left(\beta_{1 j_{2}} \cos 2 j_{2} \pi\left(\frac{t}{T}\right)+\beta_{2 j_{2}} \sin 2 j_{2} \pi\left(\frac{t}{T}\right)\right)+\sum_{p_{2}=1}^{P_{2}} \gamma_{p_{2}} h_{t-p_{2}}+\varepsilon_{2 t}
\end{aligned}
$$

Note that $e_{t}$ follows $\log \chi^{2}(1)$ distribution. Following the literature, I use a mixture of normal approximation of the $\log \chi^{2}(1)$ distribution. Specifically, I select a mixture of seven normal densities with component probability $q_{j}$, mean $m_{j}-1.2704$, and variance $v_{j}^{2}$, provided in Kim, Shephard, and Chib (1998).

The following is the Gibbs sampling procedure to sample the climate uncertainty. For the notational convenience, let's rewrite the equations (33) by

$$
\begin{aligned}
y_{t}^{* *} & =2 h_{t}+e_{t} \\
h_{t} & =F h_{t-1}+G Z_{t}+\varepsilon_{t}
\end{aligned}
$$

Here, new transition equation is expressed as companion form. First, I sample log squared residuals $y_{t}^{* *}$ from the equation (31). To do so, I exploit the first 80 training samples to obtain LS estimates and their variance. Specifically, the prior distribution of parameters of measurement equation is given by

$$
\left[\alpha, \beta_{1}, \gamma_{1}\right] \sim \mathbb{N}\left(\left(\hat{\alpha}^{L S}, \hat{\beta}_{1}^{L S}, \hat{\gamma}_{1}^{L S}\right), 4 * V\left(\hat{\alpha}^{L S}, \hat{\beta}_{1}^{L S}, \hat{\gamma}_{1}^{L S}\right)\right)
$$

The next step is to sample the parameters of transition equation with prior distribution, given by

$$
\left[\beta_{2}, \gamma_{2}\right] \sim \mathbb{N}\left(\left(\hat{\beta}_{2}{ }^{L S}, \hat{\gamma}_{2}{ }^{L S}\right), I_{n}\right)
$$


where $\left(\hat{\beta}_{2}^{L S}, \hat{\gamma}_{2}^{L S}\right)$ comes from the LS estimates from the regression with sampled initial stochastic volatility and selected fourier series. The variance parameter $Q$ is sampled from IG $(2.5,0.05)$. Now, I sample $h_{t}$ using Carter and Kohn algorithm (1994). Specifically,

\section{Prediction}

$$
\begin{aligned}
& h_{t \mid t-1}=\mathbb{E}\left[h_{t} \mid \mathcal{F}_{t-1}\right]=F h_{t-1 \mid t-1}+G Z_{t} \\
& P_{t \mid t-1}=\mathbb{E}\left[\left(h_{t}-h_{t \mid t-1}\right)\left(h_{t}-h_{t \mid t-1}\right)^{\prime}\right]=F P_{t-1 \mid t-1} F^{\prime}+Q \\
& \eta_{t \mid t-1}=y_{t}^{* *}-y_{t \mid t-1}^{* *}=y_{t}^{* *}-2 h_{t \mid t-1} \\
& f_{t \mid t-1}=\mathbb{E}\left[\left(y_{t}^{* *}-y_{t \mid t-1}^{* *}\right)^{2}\right]=4 P_{t \mid t-1}+1
\end{aligned}
$$

\section{Updating}

$$
\begin{aligned}
& h_{t \mid t}=\mathbb{E}\left[h_{t} \mid \mathcal{F}_{t}\right]=h_{t \mid t-1}+2 P_{t \mid t-1} f_{t \mid t-1}^{-1} \eta_{t \mid t-1} \\
& P_{t \mid t}=\mathbb{E}\left[\left(h_{t}-h_{t \mid t}\right)\left(h_{t}-h_{t \mid t}\right)^{\prime}\right]=P_{t \mid t-1}-4 P_{t \mid t-1} f_{t \mid t-1}^{-1} P_{t \mid t-1}
\end{aligned}
$$

Now, we sample $h_{t}$ through backward recursion process. Specifically, sample $h_{T}$ from the normal distribution with mean and variance given by the updating procedure at time $T$ above.

$$
h_{T} \mid y_{T}^{* *} \sim \mathbb{N}\left(h_{T \mid T}, P_{T \mid T}\right)
$$

and then sample $h_{t}(t=1,2, \ldots, T-1)$ recursively from

$$
h_{t} \mid y_{t}^{* *}, h_{t+1} \sim \mathbb{N}\left(h_{t \mid t, h_{t+1}}, P_{t \mid t, h_{t+1}}\right)
$$


where

$$
\begin{aligned}
\eta_{t+1 \mid t}^{*} & =h_{t+1}-F h_{t \mid t}-G Z_{t+1} \\
f_{t+1 \mid t}^{*} & =F P_{t \mid t} F^{\prime}+Q \\
h_{t \mid t, h_{t+1}} & =h_{t \mid t}+P_{t \mid t} F^{\prime} f_{t+1 \mid t}^{*-1} \eta_{t+1 \mid t}^{*} \\
P_{t \mid t, h_{t+1}} & =P_{t \mid t}-P_{t \mid t} F^{\prime} f_{t+1 \mid t}^{*-1} F P_{t \mid t}
\end{aligned}
$$

The lag length selection of a fourier series is quite obvious to capture the ENSO cycle. I only include $\left(\cos 20 \pi\left(\frac{t}{T}\right), \sin 20 \pi\left(\frac{t}{T}\right), \cos 22 \pi\left(\frac{t}{T}\right), \sin 22 \pi\left(\frac{t}{T}\right), \cos 26 \pi\left(\frac{t}{T}\right)\right.$, $\left.\sin 26 \pi\left(\frac{t}{T}\right)\right)$ in both measurement and transition equations, in order to capture 4-5 year ENSO cycle, instead of including a fourier series from lower order (i.e., $J_{1}=J_{2}=$ 10, 11, 13). ${ }^{23}$ However, I choose the length of autoregressive lags using BIC criteria $\left(P_{1}=6\right.$ and $\left.P_{2}=1\right)$. Note that $I=0$ is selected as explained before. I use 25,000 replications and base my inference on the last 10,000 replications.

As illustrated in Figure 27, the selected fourier series play an important role in understanding the SST anomaly data. Obviously, the selected fourier series are all highly significant at 5\% confidence level, indicating that there has been a clear 4-5 year ENSO cycle in the SST anomaly data. To show how the cyclical terms improve the estimates of the interest, I compare the estimated climate uncertainty from the fully specified model above with that from the model with no cyclical terms (i.e., $I_{1}=$ $J_{1}=J_{2}=0$, and $P=6$ ). In Figure 28 , the estimates of the climate uncertainty are illustrated with a black line with $68 \%$ error bands, and the estimates without cyclical terms are illustrated by a red line. The $J=0$ and $P=6$ model (red line) is provided because BIC criteria shows that $P=6$ is an optimal model under a separate equation, which has LS residuals obtained by the regression of deseasonalized SST anomaly data on the selected fourier series as a dependent variable. In addition, I provide the

\footnotetext{
${ }^{23}$ I exclude $J=12$ terms because those are insignificant at $5 \%$ confidence level.
} 
estimated climate uncertainty (ERS CU in Figure 28) generated from the FAVAR with uncertainty model (i.e., $\hat{\zeta_{1 t}}$ of equation (23)). As expected, including cyclical terms provides more reasonable estimates of the climate uncertainty. However, the climate uncertainty generated by the ENSO factor is somewhat different with that by Campbell and Diebold (2005) framework, which is sensible result.
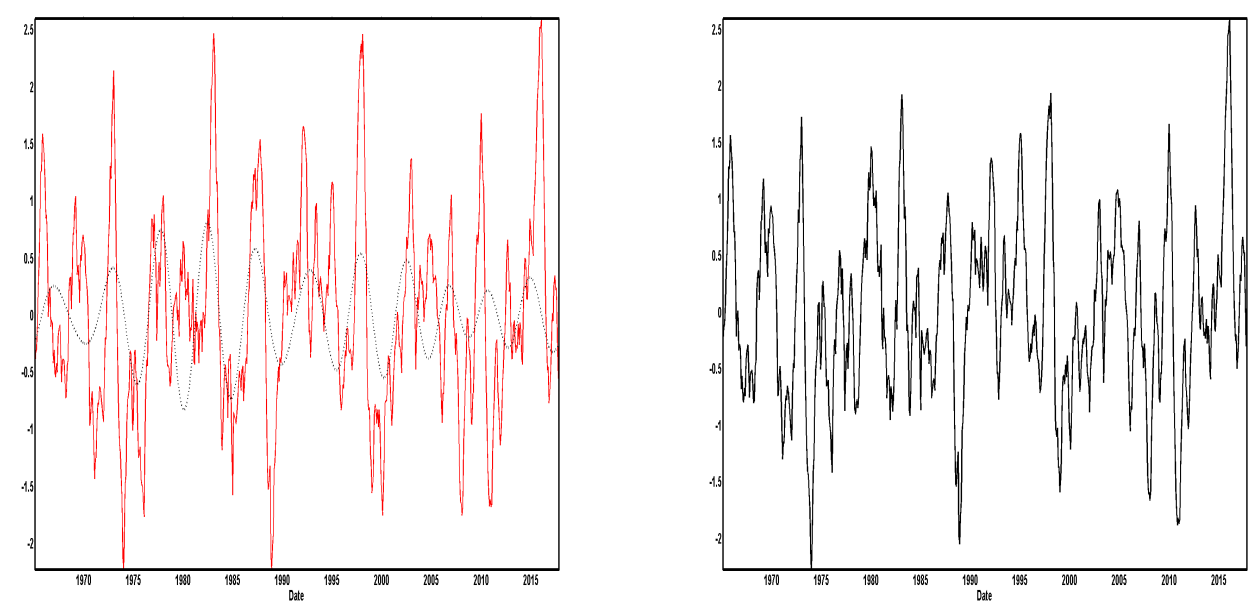

Figure 27: The 4-5 year cyclical (left) and high frequency cycle (right) components of the SST anomaly 


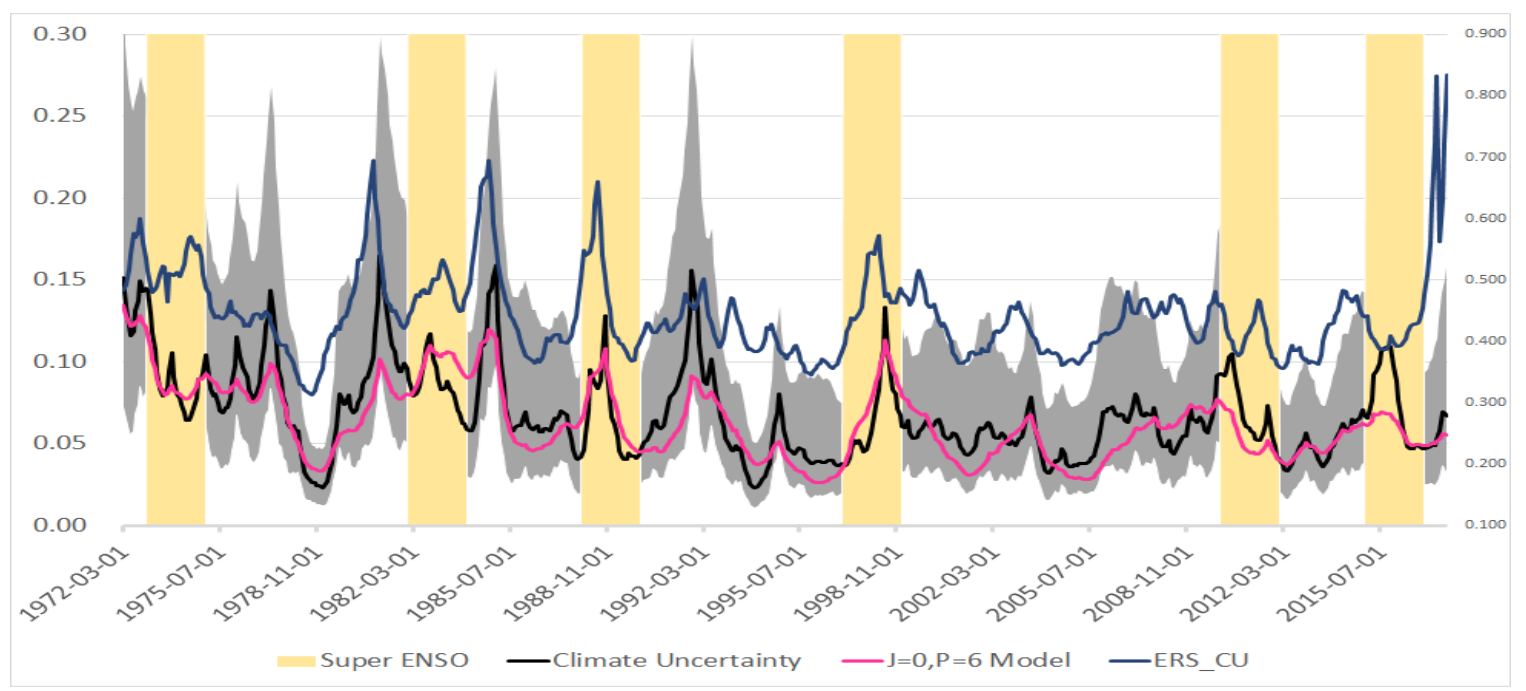

Figure 28: The estimate of climate uncertainty from the fully specified model and that from the model with no cyclical terms and that from the FAVAR with uncertainty model and ERS generated data 


\section{Unobserved Common Component Model}

To identify the role of two-state regime switching framework of the observed data, I consider the unobserved common component model as given by

$$
\begin{aligned}
y_{i t} & =\alpha+\beta x_{t}+u_{t}, \quad i=1,2 . \\
x_{t} & =\mu+F x_{t-1}+e_{t}
\end{aligned}
$$

where $y_{1 t}$ and $y_{2 t}$ are the deseasonalized SST anomaly and the deseasonalized SOI data, respectively. The two errors of the measurement and transition equations follow the normal distribution with zero mean and constant variance. That is, $u_{t} \sim$ i.i.d.N $(0, R)$ and $e_{t} \sim$ i.i.d.N $(0, Q)$. The estimation of the model (35) is implemented by the classical maximum likelihood approach with Kalman filter. Further, I assume $x_{0} \sim N(\tilde{x}, 1)$, where $\tilde{x}$ is the first observation of the static principal component.

Figure 29 compares the extracted unobserved common component (red line) with the first static principal component (dotted black line), and illustrates their sample autocorrelation. As shown, unobserved common component shows essentially same dynamics with the first static principal component, implying that ignoring the twostate regime switching framework in the observed data cannot solve the problem addressed in Section 3.

\section{Convergence Check for the MCMC Algorithm}



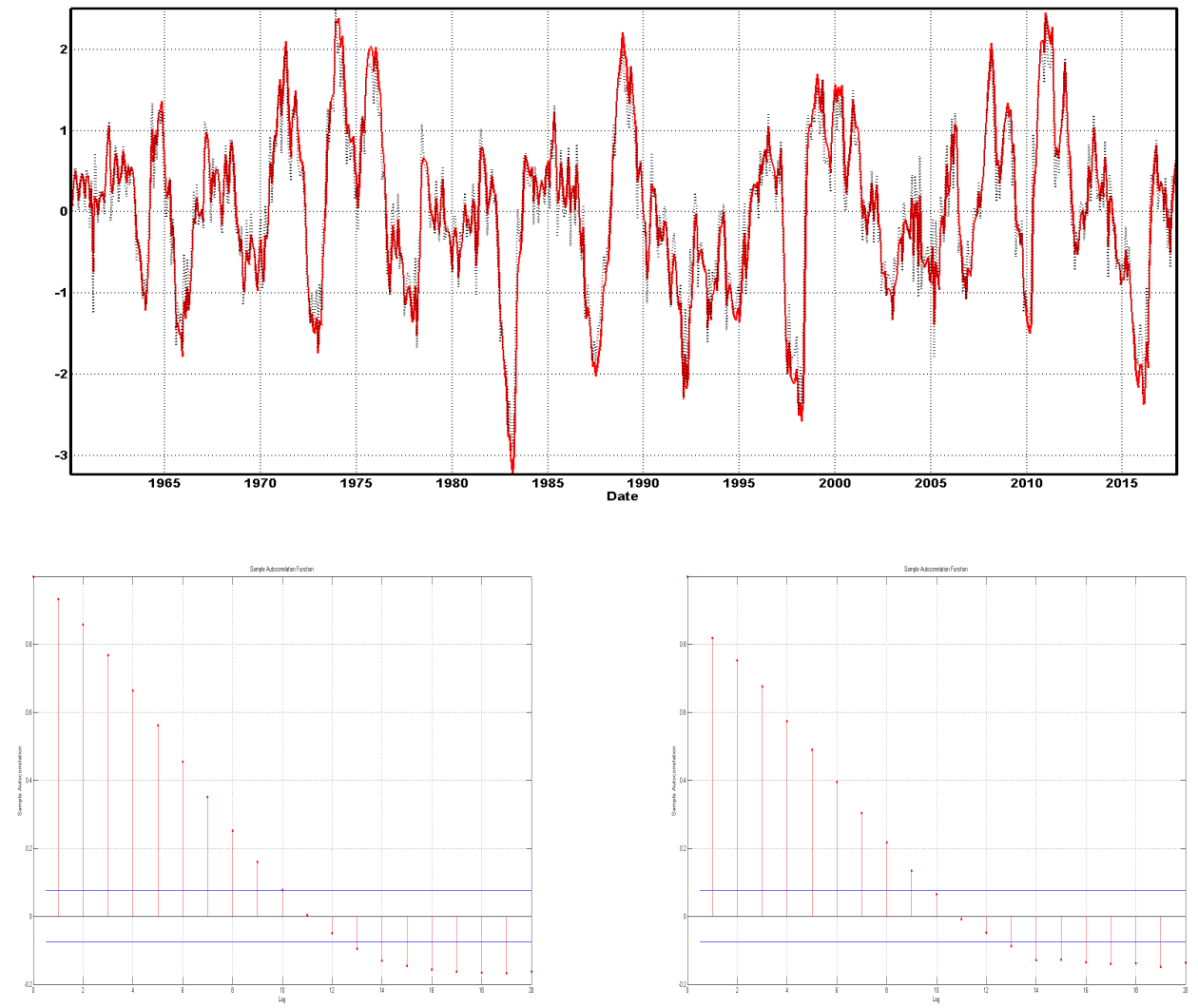

Figure 29: The unobserved component and first static principal component and their sample autocorrelation 

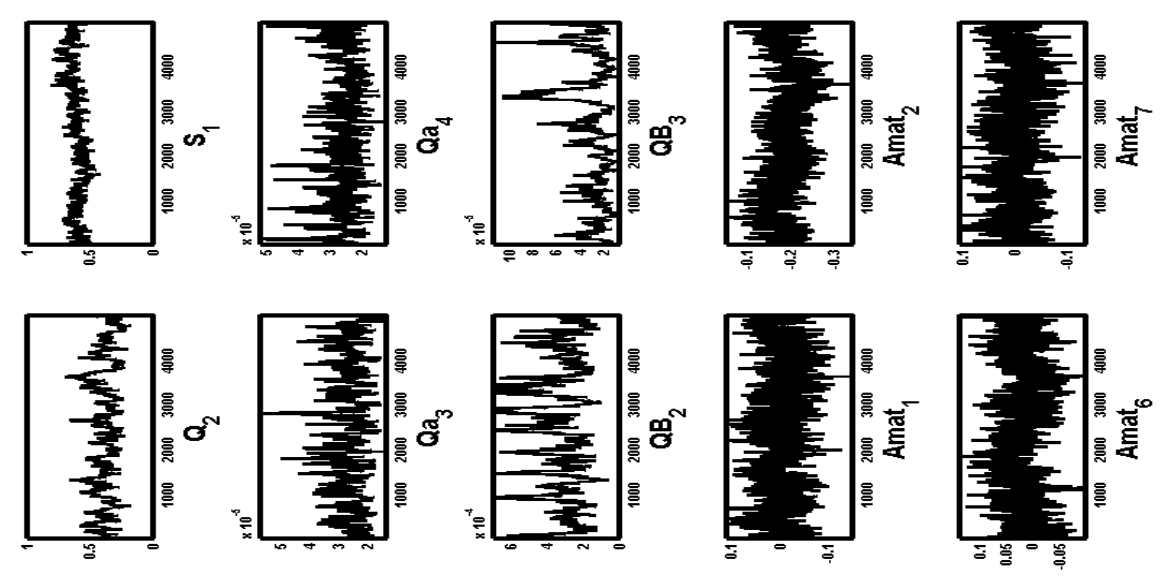

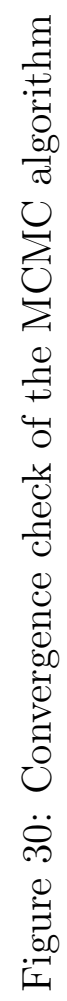

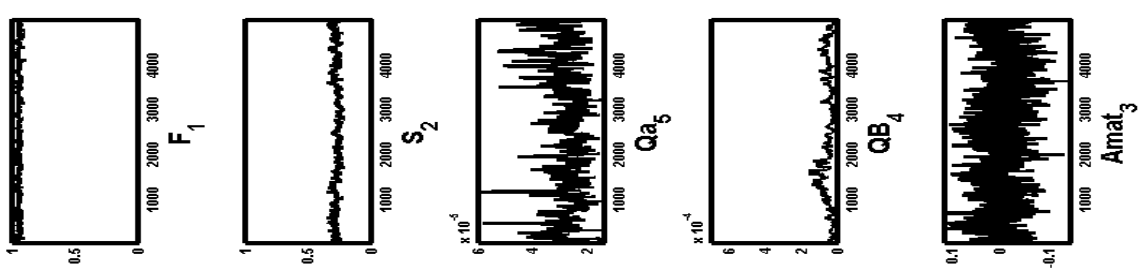




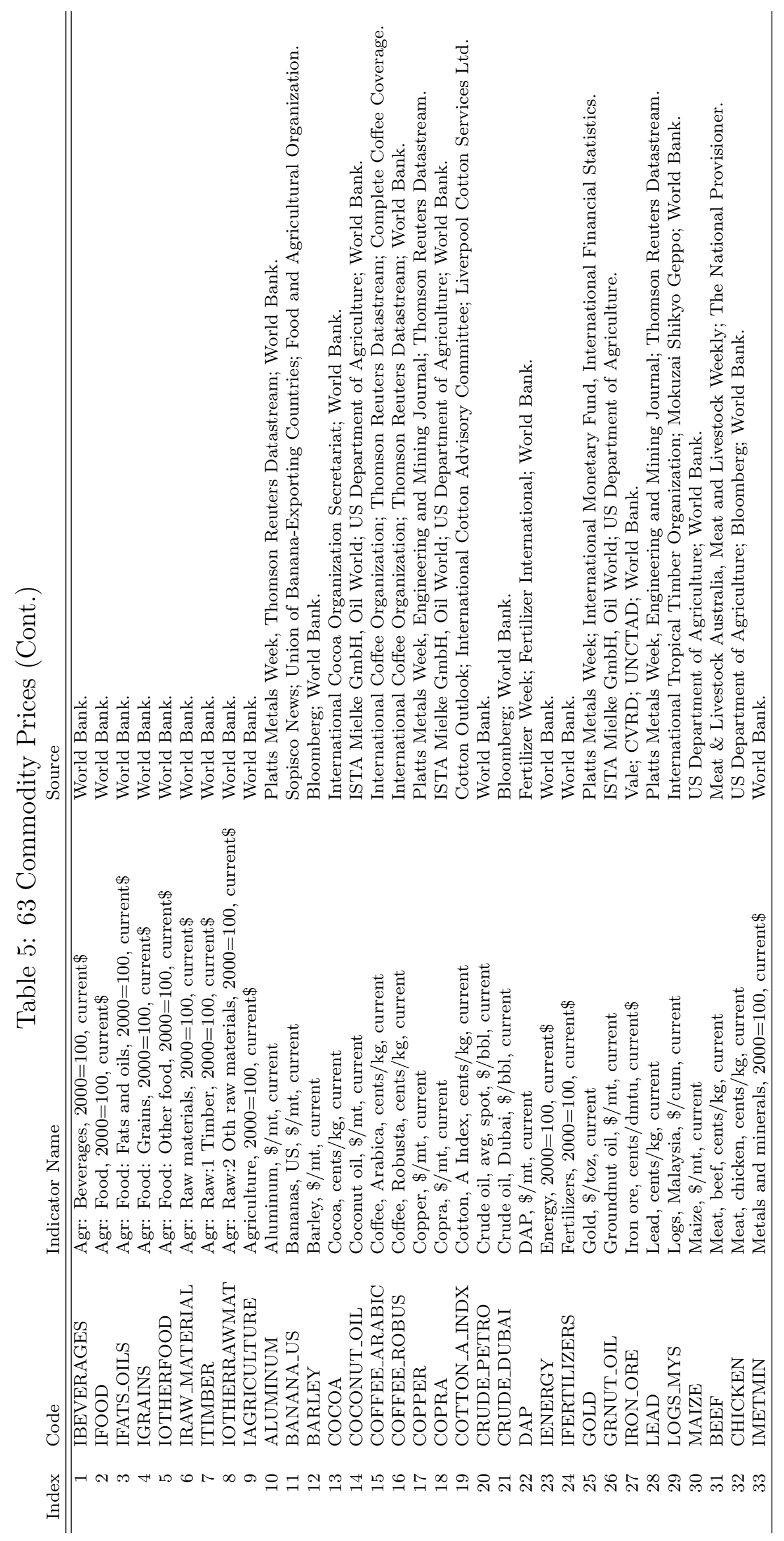

124 


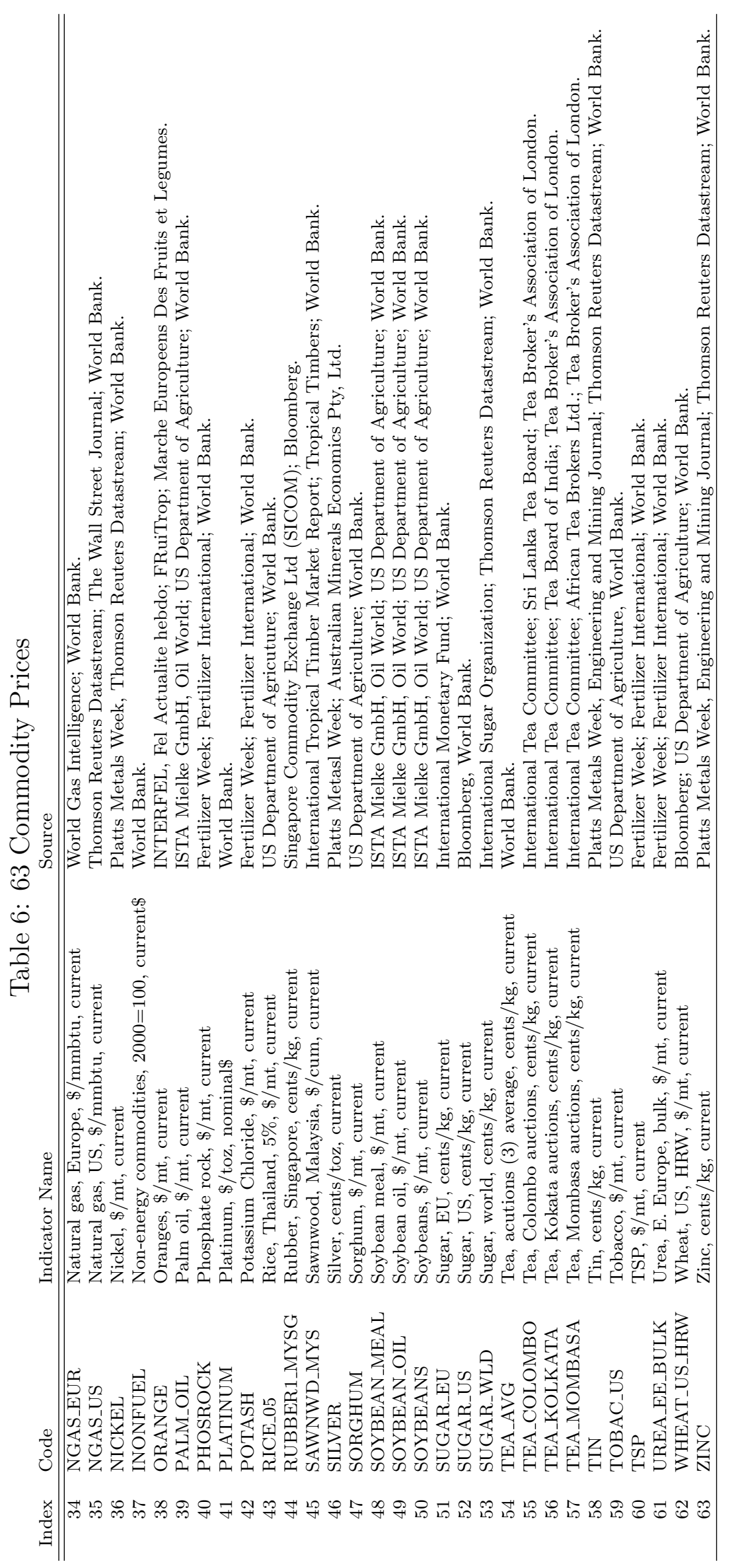

125 


\section{VITA}

Kyungsik Nam was born on July 10 th 1983 in Seoul, South Korea. He attended Sangmoon High School, Seoul, South Korea. In 2003, he entered Sungkyunkwan University in Seoul, South Korea. He graduated with a Bachelor's degree in Economics in February, 2009. He received a degree of Master of Economics from Sungkyunkwan University in August, 2011. Also, he was a member of the Center for Econometric Research (CER) in Sungkyunkwan University from 2008 to 2011. In 2012, he was employed as a research assistant at Korea Economic Research Institute (KERI). He entered the Graduate school at the University of Missouri-Columbia in August, 2012. In 2018, he received a Ph.D. in Economics from the University of Missouri-Columbia. 